\title{
Age-related UV absorption of the human eye lens and its molecular background
}

\author{
Ph.D. Thesis
}

Viktor Pajer M.Sc.

Szeged, 2020 


\title{
Age-related UV absorption of the human eye lens and its molecular background
}

\author{
Ph.D. Thesis
}

Viktor Pajer M.Sc.

Laboratory of Neural Regeneration, Department of Anatomy, Histology and Embryology, Faculty of Medicine, University of Szeged

Supervisor: Prof. Dr. Antal Nógrádi

Szeged, 2020 


\section{Original papers related to this thesis}

I. Pajer V, Tiboldi Á, Bae N, Li K, Kang SU, Hopp B, Kolozsvári L, Lubec G and Nógrádi A, The molecular background of the differential UV absorbance of the human lens in the 240-400 nm range. 2013, Photochem Photobiol, 89: 856-863. IF : 2.684

II. Pajer V, Rárosi F, Kolozsvári L, Hopp B and Nógrádi A, Age-related absorption of the human lens in the near-ultraviolet range. 2020, Photochem Photobiol, php.13199 IF: $2.338(2018)$ 


\section{Table of contents}

Summary 1

Introduction 2

Morphology of the human eye lens 2

UV irradiation as a risk factor of lens opacities 4

UV induced cataract formation 4

Photodamage $\quad 5$

Absorption characteristics of the lens 5

Absorbance spectrum of the lens $\quad 6$

UV absorption at molecular level $\quad 6$

$\begin{array}{ll}\text { Age-related changes } & 7\end{array}$

$\begin{array}{lr}\text { Aims of the study } & 8\end{array}$

$\begin{array}{ll}\text { Material and Methods } & 9\end{array}$

Handling and maintenance of eye lenses $\quad 9$

$\begin{array}{ll}\text { Cryostat sectioning } & 9\end{array}$

Spectrophotometric measurements 10

Quantification of capsule thickness 12

$\begin{array}{ll}\text { Proteomic studies } & 12\end{array}$

Two-dimensional gel electrophoresis 12

Quantification of protein levels 12

Analysis of peptides by Nano-LC-ESI-MS/MS 13

Verification of protein expression levels 13

$\begin{array}{ll}\text { Statistical analysis } & 14\end{array}$

$\begin{array}{ll}\text { Results } & 15\end{array}$

Absorption spectra of the lens sections $\quad 15$

Absorption capacity of the lens capsules 17

$\begin{array}{ll}\text { Absorption spectrum } & 17\end{array}$

Contribution of the epithelium to the capsular UV absorbance 19

Transmission of the lens 19 
Age-related changes of the UV absorption 21

Protein expression in the anterior and posterior cortex 24

$\begin{array}{ll}\text { Discussion } & 28\end{array}$

The UV absorption increases in antero-posterior direction 28

Only the posterior layers show age-dependent changes $\quad 29$

Absorption gradient is accompanied by differential protein expression levels 31

Conclusions $\quad 34$

$\begin{array}{ll}\text { Acknowledgements } & 35\end{array}$

$\begin{array}{ll}\text { References } & 36\end{array}$

$\begin{array}{ll}\text { Appendix } & 42\end{array}$ 


\section{Summary}

The human cornea absorbs most of the ultraviolet (UV) radiation, but still significant amount of UV light may reach the lens. It is well known that exposure to UV light may lead to ocular damage. Protection of the retina is even more crucial since it is more sensitive to the harmful radiations. The aim of the present study was to determine the UV absorption of various parts (nucleus, anterior- and posterior cortex) of the lens and lens capsules, to examine the age-related changes of the UV absorption and to carry out mass spectrometry on lenticular segments derived from anterior and posterior parts.

Sixty $\mu \mathrm{m}$ thick cryostat sections were cut from human lenses, absorbance spectra of the sample layers and that of the lens capsules were determined using a scanning spectrophotometer and the absorption coefficients were calculated in the $240-400 \mathrm{~nm}$ wavelength range. Two-dimensional gel electrophoresis (2DE) was performed using two pooled lenticular protein extracts. Protein spots were quantified and significantly different spots were identified by mass spectrometry. Correlation between age and the absorption coefficient values taken at $280 \mathrm{~nm}$ and at $360 \mathrm{~nm}$ was examined by statistical analysis.

Absorption spectra of the lens sections and the capsules had a peak at $280 \mathrm{~nm}$ wavelength. UV absorption of the samples taken from the lens increased anteroposteriorly regardless of the anatomical structure and age. There was a significant correlation between age and the absorption coefficient of the samples taken from the posterior part of the lens. Posterior capsules showed higher absorption coefficient than the anterior ones independently of age but the capsules showed no significant age-dependent changes. Levels of proteins Beta-crystallin B2, A3 and of glyceraldehyde-3-phosphate dehydrogenase (G3PD) were significantly higher in the anterior part of the lens, whereas proteins Beta-crystallin B1 and Alpha-crystallin A were present in higher concentration in the posterior part.

In this study we have provided evidence that the various parts of the lens have differential UV absorption capacity and this is accompanied by a differential protein expression in the anterior and posterior lens parts. UV absorption of the posterior sections varies in an age-dependent manner. Lens capsules significantly contribute to the absorption capacity of the human lens, on the other hand the age has no effect on their UV absorption. 


\section{Introduction}

\section{Morphology of the human eye lens}

The lens is a transparent, elastic, encapsulated body which has a biconvex shape: the convexity of the anterior surface is less steep than that of the posterior one (Gray, 2008). The central points of these surfaces are the poles, whereas the marginal circumference of the lens is the equator. Posteriorly, it contacts the hyaloid fossa of the vitreous body, anteriorly, it forms a ring-like contact with the iris. The lens is encircled by the ciliary processes and is attached to them by the zonular fibers which insert into the lens capsule at the equator. The muscles in the ciliary body regulate the tension exerted on the lens and thus change its shape: this process, in which the focus of the eye is adjusted, is called accommodation.

The lens consists of three layers: lens fibers compose most of the lens, the anterior surface is covered by epithelial cells and the whole is surrounded by the lens capsule. The epithelial cells differentiate into lens fibers: they undergo mitosis at a germinative zone just anterior to the equator and are displaced towards the equator where they synthesize lens fiber proteins and undergo extreme elongation. The inwardly displaced epithelial cells elongate further in both anterior and posterior direction and the lens fiber cells are laid layer upon layer. As a consequence, in the differentiated fiber cells there is no protein turnover (Horwitz, 2003). Younger fibers compose the softer cortical zone and the firmer nucleus consists of older fibers. In the human lens, at birth there are 1.6 million fiber cells, then this number increases continuously and reaches 3.5 million at the age of 80 years. The eye lens has the highest protein content inside the human body, the crystallins are the major proteins in the lens (Bloemendal et al., 2004), they occur in very high concentration, give up to $60 \%$ of the lens fiber mass and assist in maintaining the proper refractive index of the lens. At least three varieties of crystallin coexist, Alpha-, Beta- and Gamma- crystallin. The Beta- and Gamma-crystallins are referred together as Beta-Gamma-crystallin superfamily.

Alpha-crystallin has a similar structure as the small heat shock proteins (De Jong 
et al., 1988) and consists of two subunits, Alpha-crystallin A and B (Horwitz, 1999), which comprising 173 and 175 amino acid residues and with a molar ratio of 3:1, respectively (Bloemendal, 1981). The concentration of Alpha-crystallin increases with age at the expense of Beta- and Gamma-crystallins (Bron et al., 2000). It has been shown that Alpha-crystallin act as a molecular chaperone (Groenen et al., 1994; Lee et al., 1997) and can inhibit protein aggregation which can be caused, e.g., by UV irradiation (Wang and Wen, 2010). Moreover, it can also help in renaturation of G3PD (Ganea and Harding, 2000).

Beta- and Gamma-crystallins are fiber cell specific proteins. Beta-crystallins are divided into two groups: basic (B) and acidic (A) (Slingsby and Clout, 1999) and can be subdivided to seven subtypes: four of which are acidic (A1, A2, A3, and A4), and three are basic (B1, B2, and B3). Gamma-crystallins are monomeric proteins and have very similar domain structures to Beta-crystallins. So far, eight Gamma-crystallin genes have been identified in mammals: A-F, M and S (Slingsby et al., 2013).

Beside the crystallins, the human lens contains low-molecular weight compounds, also known as UV filter molecules. The four major members of this group, in order of abundance, are L-3-hydroxykynurenine O-Beta-D-glucoside (3OHKG), 4-(2-amino-3hydroxyphenyl)-4-oxobutanoic acid O-Beta-D-glucoside (AHBG), Kynurenine (Kyn) and 3-hydroxykynurenine (3OHKyn). The primate lens is unique in the sense that it synthesizes the most abundant UV filter, 3OHKG from the amino acid Tryptophan (Trp). Kyn and 3OHKyn are metabolites of Trp (Wood and Truscott, 1993) and 3OHKG is formed by 3OHKyn glycosylation (Wood and Truscott, 1994). AHBG is derived from 3OHKG (Truscott et al., 1994) through a deamination-reduction process (Bova et al., 1999). Concentration of Kyn and 3OHKG covalently bound to lens proteins are significantly higher in the nucleus than in the cortex (Korlimbinis et al., 2007). On the other hand, 3OHKyn levels show an inverse tendency (Bova et al., 2001). The concentration of free UV filters present in the older nucleus is approximately equal to that of the bound filters and the level of all these compounds show a decreasing tendency with age, except the glutathione adduct of $3 \mathrm{OHKG}$.

The capsule is the thickest basement membrane in the body, appears dense and ho- 
mogeneous under the microscope (Alió et al., 2008). It consists of glycosaminoglycans, glycoproteins and various classes of collagen fibers. The major collagen in the capsule is type IV collagen. Ultrastructurally, the capsule is a flexible, three-dimensional molecular network formed by the interaction of type IV collagen and laminin networks with the help of other proteoglycans, such as nidogen (Danysh and Duncan, 2009). The anterior lens capsule is synthesized by the lens epithelium and reflects the epithelial cell activity. It continuously grows throughout the life, its thickness at the anterior pole increases from 15 to about $25 \mu \mathrm{m}$ (Krag et al., 1997). The posterior capsule is 3-5 times thinner (Krag and Andreassen, 2003) and loses its epithelial cells in fetal life. Its lamellar structure also disappears much earlier than that of the anterior capsule. It is still unclear whether the posterior capsule is synthesized by the nucleated cortical lens fibers or by anterior epithelial cells, whereupon it is secreted into the posterior aspects of the lens.

\section{UV irradiation as a risk factor of lens opacities}

UV is the 10-400 nm wavelength range of the electromagnetic spectrum which can be divided into extreme UV (10 to $120 \mathrm{~nm}$ ), far UV (120 to $200 \mathrm{~nm}$ ), UV-C (200 to 280 $\mathrm{nm})$, UV-B (280 to $315 \mathrm{~nm})$, and UV-A (330 to $400 \mathrm{~nm})$. UV-A and UV-B ranges are often referred together as near-UV range. Although, most of the UV light from the sun is absorbed in the atmosphere, even so significant amount of the radiation gets to the earth's surface, thus to the human eye, too. Irradiation from artificial UV sources can also reach the eye.

\section{UV induced cataract formation}

For a long while, scientists have been interested in the relationship between sun light and the risk of eye diseases. It is well known that the exposure to UV light may cause ocular damage (Merriam, 1996; Pitts et al., 1977; Zigman, 1993) and long term UV irradiation, even at the subsolar level, leads to pathological changes in the mammalian eye lens (Kessel et al., 2011; Meyer et al., 2014; Zigman, 1995; Zigman et al., 1991). These experimental results obtained under laboratory conditions unambiguously indicate the harmful effect of UV light. Population-based studies can give a more comprehensive picture about the relation between sun exposure and lens opacity formation. Several statistical data provide 
evidence for the risk of eye diseases related to the solar UV radiation (Hollows and Moran, 1981; Roberts, 2001; Taylor, 1980; Zhu et al., 2015), especially for that of different types of cataract. Development of posterior subcapsular cataract is significantly associated with the higher exposure to UV light (Bochow et al., 1989). Higher prevalence of cortical opacities is also related to UV-B irradiation (Cruickshanks et al., 1992; West et al., 1998). Sun exposure can cause nuclear cataract in the young lens (Neale et al., 2003) but no further association has been found between the sun light and nuclear opacities formation (AREDS, 2001; Dolin, 1994; Taylor et al., 1988). Incidence of both nuclear and cortical cataracts increases with age (Klein et al., 1998).

\section{Photodamage}

Any types of damage in the lens compounds result in the change of the refractive index and can lead to opacification. One of the environmental factors generating oxidative damage of lens constituents is near-UV radiation. Zigman et al. found (1973) that the UV induced lens pigmentation can be explained by photo-oxidation of aromatic amino acids. Pirie (Pirie, 1971) showed that when free Trp or proteins containing Trp are exposed to direct sunlight N'-Formylkynurenine (N-FK) is formed and loss of Trp can be observed. Formation of N-FK may be essential for the production of $\mathrm{H}_{2} \mathrm{O}_{2}$ in irradiated Trp which process is involved in the photooxidation of Alpha-crystallin (Andley and Clark, 1989). Trp fluorescence in the irradiated proteins decreases exponentially with the UV dose, indicating photochemical destruction (Löfgren, 2017). UV irradiation can also destroy the secondary structure of Alpha-crystallin (Fujii et al., 1999) and reduce its chaperone efficiency (Cetinel et al., 2017), especially in the case of Alpha-crystallin A (Weinreb et al., 2000). Oxidative stress markers were found in mice lenses exposed to UV-A and UV-B radiation. G3PD activity, which is an important component in the glycolytic pathway to produce ATP in the lens, is also significantly decreased in cataractous mice lenses (Zhang et al., 2012).

\section{Absorption characteristics of the lens}

The main function of the lens is to focus the incoming visible light to the retina, meanwhile the crystallins and lens fibers support the proper transmission by providing the necessary 
refractive index. Beyond this role, the lens is also an important protective segment inside the eye. Since the retina is more sensitive to the UV light, even a small amount of irradiation can damage the photoreceptors. The transmission of the eye lens decreases rapidly at wavelengths shorter than $400 \mathrm{~nm}$ (Boettner and Wolter, 1962) and thus it helps to prevent the retina from the harmful optical radiations.

\section{Absorbance spectrum of the lens}

In the near-UV range, the exact characteristic of the absorbance spectrum of the lens is well-known long ago (Cooper and Robson, 1969): below $400 \mathrm{~nm}$ the absorption increases towards to the shorter wavelengths and has a peak at $360 \mathrm{~nm}$. From the wavelength of $360 \mathrm{~nm}$ to $320 \mathrm{~nm}$ it decreases, then in the UV-B range an increasing tendency can be observed again. Nevertheless, no data are available regarding to the UV-C range.

It has been shown that the distinct parts of the eye absorb the UV light in a different manner (Boettner and Wolter, 1962) and the various layers of the cornea show different absorption capability (Kolozsvári et al., 2002). Available data in the literature related to the absorption properties of the various layers of the lens are very limited and only a small number of human samples were investigated. The UV absorbance of nucleus is higher than the outer cortex (Dillon et al., 1999) and absorbance spectra of thin lens segments with equal thickness show that the absorbance increases anteroposteriorly (Gaillard et al., 2000). On the other hand, no absorption data are available related to the posterior cortex and very little is known about the absorption capacity of the lens capsules which likely express similar absorption characteristics as the lens (Murata, 1987; Söderberg et al., 1996).

\section{UV absorption at molecular level}

Amino acids and proteins present in the lens are effective UV filters (Coulter et al., 1936; Goldfarb et al., 1951; Rosenheck and Doty, 1961). The absorption spectrum of Trp shows a peak at $280 \mathrm{~nm}$ in the 220-310 $\mathrm{nm}$ wavelength range (Holiday, 1936) and below 245 $\mathrm{nm}$ the absorption increases sharply towards to the shorter wavelengths. Crystallins are certainly involved in UV absorption and mainly responsible for the absorption peak at $280 \mathrm{~nm}$ since their spectra are very similar to Trp (Liang et al., 1985a,b; Zelentsova et al., 
2017). The Trp derived UV filter compounds also show higher absorption in the UV-B and $-\mathrm{C}$ ranges than in the UV-A and thus significantly contribute to the UV absorption capacity of the lens (Dillon, 1991; Vazquez et al., 2002). As their absorption is considerably less than the proteins, their role is mainly providing the absorption at $360 \mathrm{~nm}$.

\section{Age-related changes}

It is well-known that the human vision impairs with age, the accommodation function decreases as the lens fibers lose their flexibility. Beside this, the lens becomes more opaque: transmission spectra of human lenses of different age show that the old lens transmits less amount of light both in the visible and in the near-UV range (Artigas et al., 2012; Boettner and Wolter, 1962). In accordance with the reduced transmission, it has been shown that the UV absorbance of the aged lens is increased compared to the young one (Weale, 1988). Interestingly, other studies found (Cooper and Robson, 1969; Gaillard et al., 2000) that the absorption peak at $360 \mathrm{~nm}$ shifts towards the shorter wavelengths with age and the band at $320 \mathrm{~nm}$ is less observable. On the other hand, the measured transmission is always increased around $320 \mathrm{~nm}$, independently of age, except in the study of Ambach et al. (Ambach et al., 1994). The above data do not provide any information about the age-related UV absorbance of the different parts of the lens and no further data are available related to the UV-C range.

Mellerio suggested (Mellerio, 1987) that the increased absorbance of the older lenses occurs due to two processes: as the lens continuously grows, the light path is longer and thus the absorbance of the entire lens is higher. Another reason is the increased pigment deposition in the nucleus. Previously, it had been shown that the human lens becomes yellow with age (Cooper and Robson, 1969) and the absorbance spectrum of the yellow pigment shows similar features as that of the lens. This age-related yellowing can be explained by the interaction between UV filter compounds and proteins (Hood et al., 1999; Vazquez et al., 2002). 


\section{Aims of the study}

In this study we intended

1. to determine the UV absorption capacity of the various parts of the lens and of the lens capsules,

2. to analyse the effect of age on the UV absorption of the different layers of the lens and of the lens capsules.

3. and to study the molecular background of the UV absorption of the anterior and posterior lenticular segments. 


\section{Material and Methods}

\section{Handling and maintenance of eye lenses}

Human lenses ( $\mathrm{n}=44$, from age of 7 to 81 years) were obtained from eyes with a postmortem delay of maximum 6 hours. The eye balls were transported and stored in a humid chamber at $+4{ }^{\circ} \mathrm{C}$ until the lenses were removed. After removal the lenses were stored at $-70{ }^{\circ} \mathrm{C}$, until further use. Only those lenses which showed no cataract formation upon post-mortem examinations were used for measurements. To facilitate the sectioning, the lenses were placed into cold $30 \%$ sucrose in Phosphate Buffered Saline (PBS) the day before and stored at $+4{ }^{\circ} \mathrm{C}$.

\section{Cryostat sectioning}

Before sectioning, the lens capsules were removed and stored in physiological saline separately. Altogether 18 anterior and 27 posterior capsules were used for spectrophotometric measurements. One additional anterior capsule was used after trypsin treatment to measure its absorbance without the epithelial layer. Sixty $\mu \mathrm{m}$ thick consecutive sections were cut from the lenses $(\mathrm{n}=38)$ on a cryostat (Leica CM1850, Leica GmbH, Nussloch, Germany) at $-18{ }^{\circ} \mathrm{C}$. During sectioning, the anterior $200 \mu \mathrm{m}$ thick portion of the lens was discarded, then the first sample was taken from $200 \mu \mathrm{m}$ anteroposteriorly. The next two $60 \mu \mathrm{m}$ thick slices were not used and the second section was taken posterior to these. In this way, we had two samples from anterior cortex. Posterior to that, every seventh section was kept, therefore five samples were taken from the nucleus. The remaining part of the nucleus was not used. Two further samples were taken from the posterior cortex with a $120 \mu \mathrm{m}$ thick discarded portion between them. Altogether, nine 60- $\mu \mathrm{m}$-thick sections were taken for UV absorption measurements in an anteroposterior order: two from the anterior cortex, five from the nucleus and two from the posterior cortex. Figure 1 shows the position of the samples within the lens. As a result of the sectioning method, samples 4-7 always derived from the juvenile nucleus (Augusteyn, 2010; Rosen et al., 2006; Taylor et al., 1996), thus the age-related changes of the sections taken from the nucleus are comparable, despite the fact that the thickness of the full lens increases with age. To 


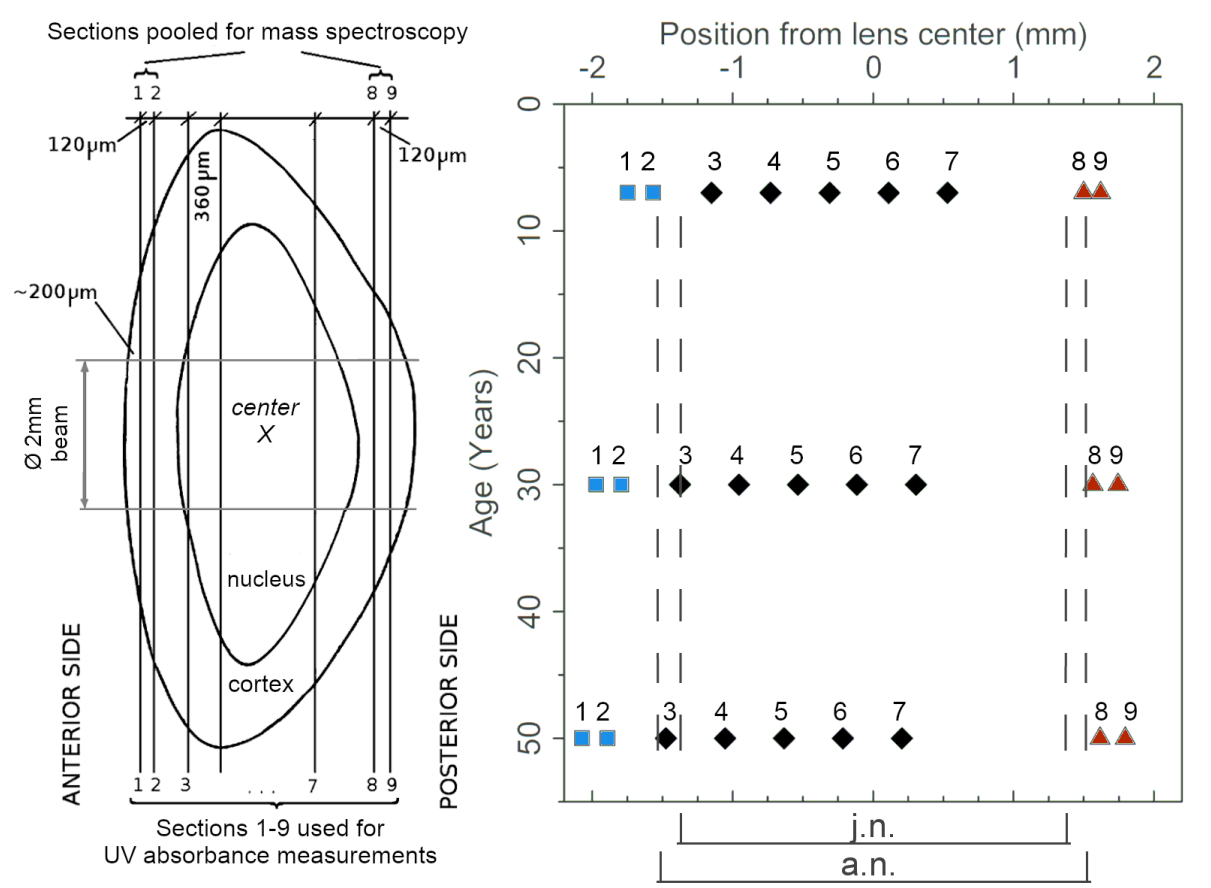

Figure 1. Left: The position of the different sample layers within the lens. Layers 1 and 2 were taken from the anterior cortex, the next five (3-7) from the nucleus and the samples 8 and 9 derived from the posterior cortex. From six additional lenses, layers 1 and 2 as well as 8 and 9 were used for proteomic studies. Right: Calculated position of sample layers from the center. Different nuclear parts of an aged lens are indicated based on literature data. j.n.: juvenile nucleus, a.n.: adult nucleus.

avoid the light scattering during the spectrophotometric measurements, the sections and the lens capsules were placed between two quartz plates with a drop of isotonic saline on both sides of the specimen. A $60 \mu \mathrm{m}$ thick spacing ring was placed between the plates to prevent compression damage to the samples. The plates were tied with a clamp spring. Cryostat sections from additional six lenses (between the age of 40 and 59) were cut in the same way for proteomic studies. Only sample layers 1, 2, 8 and 9 were kept and used as described below (2DE).

\section{Spectrophotometric measurements}

The basis of the absorption spectrophotometry is the Beer-Lambert law. According to this, in an optically homogeneous medium the intensity of the monochromatic beam, 
propagating in a given direction, exponentially decreases with the beam path:

$$
I=I_{0} e^{-\alpha d}
$$

where $I_{0}$ is the intensity of the incident light, $I$ is the intensity of the transmitted light, $e$ is the base of natural logarithm, $\alpha$ is the absorption coefficient and $d$ is the beam path. The quantity of the absorbed light can be described by the transmission $(T)$ or by the absorbance $(A)$ :

$$
\begin{gathered}
T=\frac{I}{I_{0}} \\
A=\log _{10}\left(\frac{I_{0}}{I}\right)=\log _{10}\left(e^{\alpha d}\right)
\end{gathered}
$$

The UV absorbance of the samples was measured by a scanning spectrophotometer. In the spectrophotometer, the different wavelengths are separated by a monochromator. As the beam travels through the sample, the intensity changes can be measured as function of wavelength. The model (UV-2101PC UV-VIS, Shimadzu, Kyoto, Japan) used in the experiments contains two spectral lamps (halogen lamp for 340-900 nm, and a deuterium lamp for the 190-340 $\mathrm{nm}$ wavelength ranges), a double monochromator to select the proper wavelengths, measuring and reference beam paths and a photomultiplier to detect the measuring and reference light intensities. An aperture with $2 \mathrm{~mm}$ diameter was inserted into the beam paths and the instrument was calibrated before the measurement. By applying the aperture, only the absorbance of the sample's central portions was determined and the effect of the peripheral parts was excluded. On the reference side, a drop of isotonic saline was placed between two quartz plates with a $60 \mu \mathrm{m}$ thick ring. The absorbance of the $30 \%$ sucrose in PBS (60 $\mu$ m thick layer) was determined previously and was found negligible. The spectrophotometer was connected to a computer and the absorption spectra of the samples were recorded using the software of the spectrophotometer. The investigated wavelength range was $200-400 \mathrm{~nm}$. Below $240 \mathrm{~nm}$, the measured absorbance values often approached or reached the upper limit of the device, therefore data are presented only in the 240-400 $\mathrm{nm}$ range. The absorbance is not a characteristic material constant, therefore absorption coefficient was calculated by rearranging equation 
$(3)$ :

$$
\alpha=\frac{A}{\log _{10}(e) \times d}
$$

where $d$ is the thickness of the sample.

\section{Quantification of capsule thickness}

Because the thickness of the lens capsules is not constant, it was determined for each lens. Cryostat cross sections from the samples were cut after the spectrophotometric measurements. The sections were mounted on a glass slide and were stained with hematoxylineosin: first, they were washed in distilled water, then were placed into hematoxylin for 5 min. After that, slides were placed into tap water for $10 \mathrm{~min}$ and were rinsed in distilled water. It was followed by 1 min long staining in eosin. Finally, the samples were dehydrated in an ascending alcohol series and in the end they were dipped into xylol for $2 \times$ $5 \mathrm{~min}$. The samples were coverslipped to protect them from further damage. Capsular thickness was determined by using a microscope (Olympus, Tokyo, Japan): the thickness of anterior capsules varied from $21 \mu \mathrm{m}$ to $26 \mu \mathrm{m}$, whereas that of the posterior ones were between $8 \mu \mathrm{m}$ and $11 \mu \mathrm{m}$.

\section{Proteomic studies}

\section{Two-dimensional gel electrophoresis}

Cryostat sections were cut from six lenses as described above and the anterior lenticular pool of sections consisted of sections 1 and 2, the posterior lenticular pool consisted of sections 8 and 9 . These pools were stored at $-80{ }^{\circ} \mathrm{C}$ until gel-based proteomic studies. 2DE was performed essentially as reported previously (Diao et al., 2007; Langen et al., 1999; Winding et al., 2011).

\section{Quantification of protein levels}

Excess dye from 2DE gels were removed by washing with distilled water and gels were scanned with an Image Scanner (Amersham Pharmacia Biotech). Protein spots from each gel (six gels per group, $\mathrm{n}=12$ ) were outlined (first automatically and then manually) and quantified using the Proteomweaver ${ }^{T M}$ software (Bio-Rad, CA, USA). The percentage of 
the volume of the spots representing a certain protein was determined in comparison with the total proteins present in the 2DE gel (Chen et al., 2006).

\section{Analysis of peptides by Nano-LC-ESI-MS/MS}

Fourteen spots which showed different levels between group 1 (anterior pool) and group 2 (posterior pool) were manually excised and placed into $1.5 \mathrm{~mL}$ Protein LoBind Eppendorf tubes. In-gel digestion and sample preparation for HCT analysis was performed as described by Chen et al. (Chen et al., 2006). The extracted peptides were pooled for Nano-LC-ESI-CID/ETD-MS/MS analysis as reported (Kang et al., 2011). Protein identification and modification information returned from MASCOT were manually inspected and filtered to obtain confirmed protein identification and modification lists of CID MS/MS and ETD MS/MS. Posttranslational modification (PTM) searches were done using ModiroTM v1.1 software (Protagen AG, Germany). Protein identification and modification information returned were manually inspected and filtered to obtain confirmed protein identification and modification lists (Kang et al., 2011).

\section{Verification of protein expression levels}

Each protein spot was cut out and put into a $0.5 \mathrm{~mL}$ Protein LoBind tube (Eppendorf, Hamburg, Germany). Spots were initially washed twice with ACN: de-ionized water (1:1, v/v) for 20 min. Subsequently $100 \mu \mathrm{L}$ of $100 \%$ ACN was added to each tube to cover the gel piece completely and incubated for at least 2 min. After the removal of ACN, gel pieces were dried completely in a SpeedVac Concentrator 5301. The dried gel pieces were reswollen with $60 \mu \mathrm{L}$ of $2 \mathrm{ng} / \mu \mathrm{L}$ proteinase $\mathrm{K}$ (Promega, Madison, WI, USA) buffered in $1 \mathrm{mM} \mathrm{HCl}$. Gel pieces were incubated for longer than $4 \mathrm{~h}$ at $37{ }^{\circ} \mathrm{C}$. Supernatants were transferred to new $0.5 \mathrm{~mL}$ tubes, and gel pieces were extracted by $80 \mu \mathrm{L}$ of $1 \%$ formic acid (Fluka, Steinheim, Germany) under sonication for 30 min. Following this extraction step, peptides were eluted from the gel pieces by $20 \%$ ACN under sonication for $30 \mathrm{~min}$ and subsequently by $40 \% \mathrm{ACN}$ for $30 \mathrm{~min}$. The individual extractions were pooled into the $0.5 \mathrm{~mL}$ LoBind tubes containing the supernatant including proteinase K. The volume was reduced to a final volume $<10 \mu \mathrm{L}$ in a SpeedVac concentrator and undergoing acid hydrolysis and ninhydrin reaction. Ninhydrin reaction was carried out 
as follows: Samples were hydrolyzed in $0.5 \mathrm{~mL}$ Eppendorf Protein LoBind tubes with $150 \mu \mathrm{L}$ of $6 \mathrm{~N}$ hydrochloric acid solution (Fluka) at $99{ }^{\circ} \mathrm{C}$ for $24 \mathrm{~h}$. Hydrolysates were spun down and evaporated in a SpeedVac concentrator (Eppendorf). The dried material was redissolved in $20 \mu \mathrm{L}$ of distilled water, vortexed and centrifuged. Ninhydrin working solution was prepared as referenced (Kang and Lubec, 2011). Absorption was measured on an iMark microplate reader (Bio-Rad) at $570 \mathrm{~nm}$. Measurement of all the samples was performed within 10 min.

\section{Statistical analysis}

Descriptive statistics were calculated for each layer separately. Continuous variables were expressed as mean $\pm \mathrm{SD}$. The tendency between age and absorption coefficients at $280 \mathrm{~nm}$ and at $360 \mathrm{~nm}$ was characterized through the use of Pearson correlation coefficients and significance tests for correlation for each layer separately. Analysis of covariance (ANCOVA) model with factor layer and covariate age was used for further statistical analysis. A p-value $<0.05$ was considered to be statistically significant. The calculations were computed through the use of IBM SPSS version 24.

Statistical analysis to reveal between-group differences was performed by the paired Student's t-test. Bonferroni-Holm and LSD correction was applied for correction of multiple testing. In all proteomic studies, a probability level of $\mathrm{P}<0.01$ was considered statistically significant. All calculations were performed using SPSS version 14.0 (SPSS Inc., Chicago, IL). 


\section{Results}

\section{Absorption spectra of the lens sections}

The UV absorption of the lens sections was examined in seven different age groups: 7-19 years, 20-29 years, 30-39 years, 40-49 years, 50-59 years, 60-69 years and 70-81 years. Only one lens was available under the age of 10 and thus the youngest group was complemented with this sample. For similar reasons, an 81-year-old lens was added to the last group. In this way, the number of lenses were $3,3,6,10,9,4$ and 3 respectively in the corresponding groups, described above. The absorption coefficients were calculated from the measured absorbance by using the equation (4). In each group, the averaged values were calculated and are shown as function of wavelength in Figure 2. In the UV-A range (330-400 nm), the absorption coefficients of the young lenses are very low, $<0.011 / \mu \mathrm{m}$. At ages higher than 40, the absorption is higher in this range and shows an increasing tendency, which continues in the UV-B range too. In the $310 \mathrm{~nm}$ to $240 \mathrm{~nm}$ range, the absorption coefficient steeply increases irrespectively of age and reaches a maximum at $280 \mathrm{~nm}$ (end of UV-B range). From this wavelength to $250 \mathrm{~nm}$ the absorption coefficient sharply decreases and then increases again. The samples, which were taken from more posterior parts of the lens, show the same characteristics, but the absorption coefficients of the posterior layers are higher. The same tendency can be observed in each age group, however, the peak at $280 \mathrm{~nm}$ is sharper in the older groups and at the same time the valley at $250 \mathrm{~nm}$ is more pronounced. These results clearly show that the UV absorption capacity of the lens increases anteroposteriorly, independently of the anatomical structure. In the youngest group the increasing trend is not explicit and the absorption coefficients of sections 4-7 are almost equal and sample 9 shows very low absorption compared with the other groups. It is also evident that the older lenses have higher absorption coefficients compared to the younger ones.

The reported absorbance spectra of the human lens show another minor absorption peak around 360 nm (Cooper and Robson, 1969; Gaillard et al., 2000; Weale, 1988). Although, the absorption coefficients at this wavelength is significantly lower than that of at $280 \mathrm{~nm}$, 

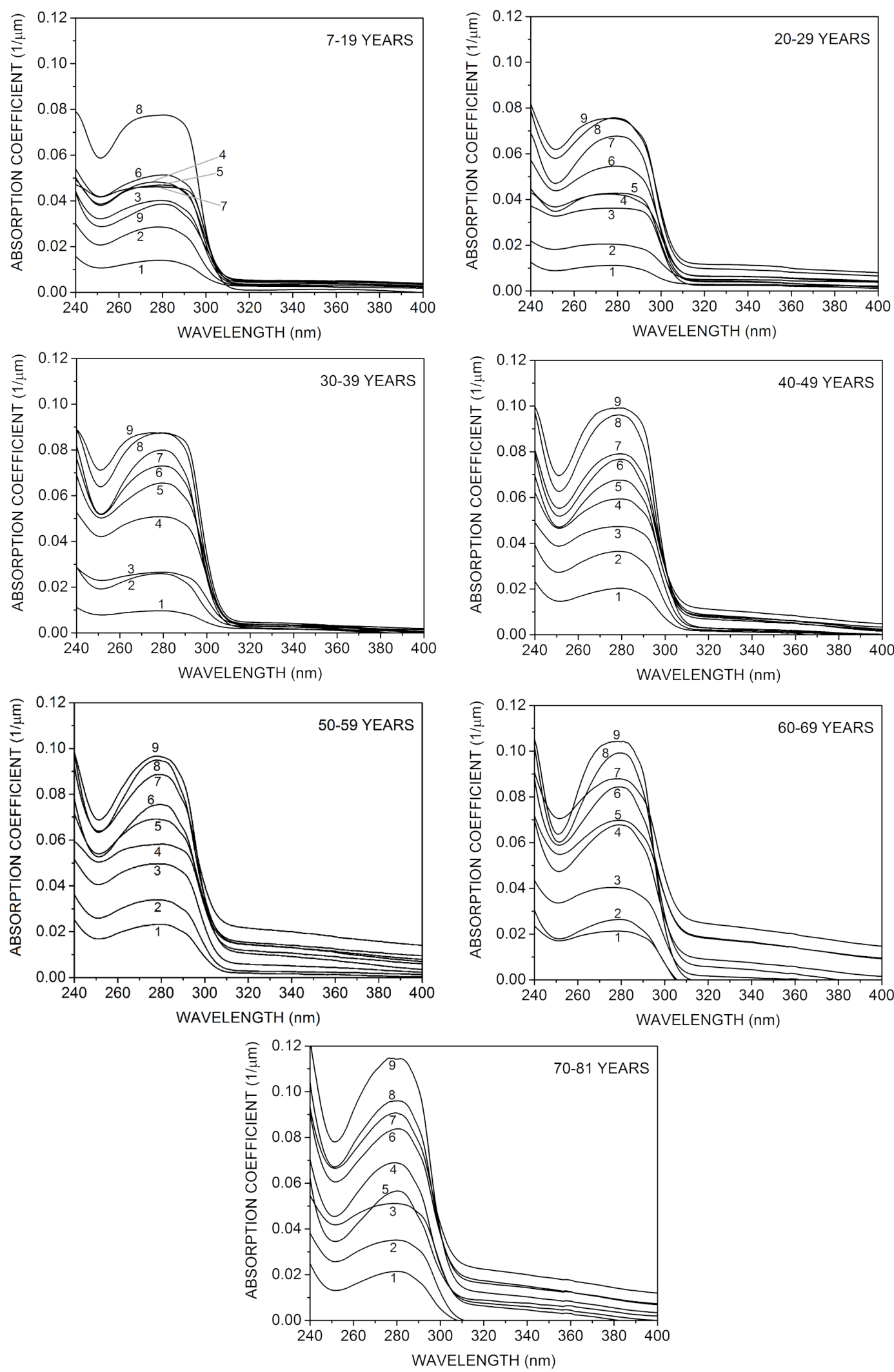

Figure 2. Averaged absorption coefficients of the nine investigated layers as a function of wavelength. Age groups are indicated on the related graphs. 
it is worth to examine the absorption in the UV-A range in more detail. Figure 3 presents the averaged absorption coefficients of three age groups in the 300-400 nm wavelength range. For clarity, data related to samples 1, 3, 5, 7 and 9 only are shown. The posterior layers have higher absorption capacity, but the increasing tendency is not as explicit as in the case of the peak at $280 \mathrm{~nm}$. As the detection limit of the spectrophotometer was calibrated in a way to measure the peak at $280 \mathrm{~nm}$, the absorbance in the UV-A range presumably could have been detected with lower precision. Therefore the fine absorption changes are visualized to a lesser extent compared with the previously published results where the investigation was focused on the peak at $360 \mathrm{~nm}$. It is noticeable that only the spectra of the youngest lenses show a small peak around $360 \mathrm{~nm}$ while the absorption is slightly decreased at $320 \mathrm{~nm}$. This is in good agreement with the data found in the literature: the peak at $360 \mathrm{~nm}$ shifts towards the shorter wavelengths with age and the minimum vanishes.
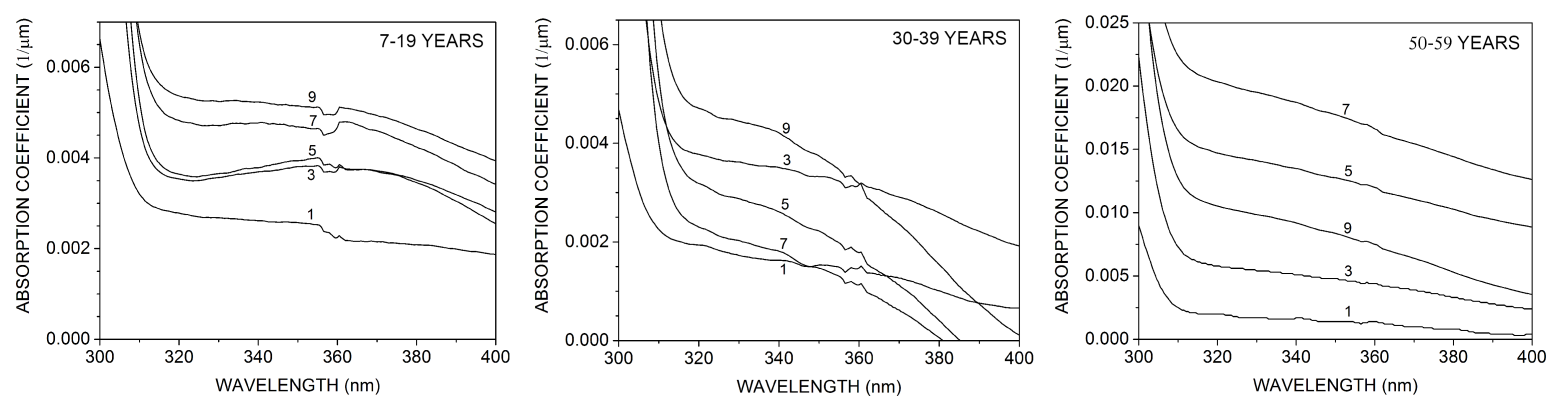

Figure 3. Averaged absorption coefficients as a function of wavelength in the 300-400 nm wavelength range. Age groups are indicated.

\section{Absorption capacity of the lens capsules}

\section{Absorption spectrum}

Some of the capsules were damaged while they were removed and thus the number of the available samples was less than that of the lenses. Between the age of 30 and 39, capsules were separated successfully from only one lens and thus the groups of 20-29 years and of 30-39 years were merged. The two oldest groups were combined, too for similar reasons. In this way, the absorption of the lens capsules was examined in five different age groups: 7-19 years, 20-39 years, 40-49 years, 50-59 years and 60-81 years. The number of the 

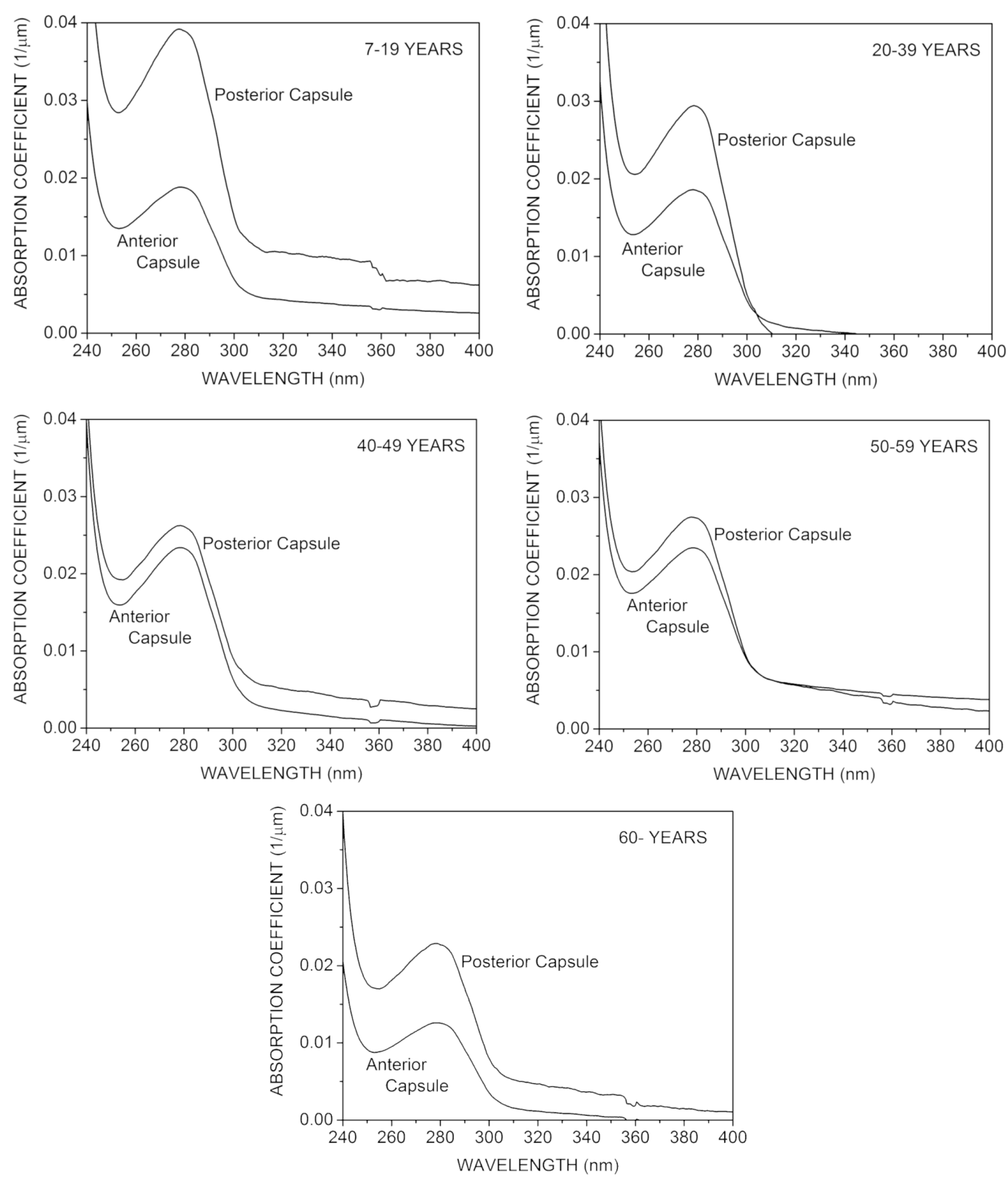

Figure 4. Averaged absorption coefficients of the capsules as a function of wavelength. Age groups are indicated on the related graphs.

anterior capsules was 2, 2, 8, 4, 2 in the relevant groups and the amount of posterior capsules were $4,3,9,8$ and 3 , respectively. The averaged absorption coefficients were calculated in a similar way as in the case of the lenses and show the same characteristics. The UV absorption is more effective in the UV-B and UV-C ranges than in UV-A range, with a maximum at $280 \mathrm{~nm}$ (Figure 4). It can be also noticed that independently of age, the posterior capsules have considerably higher absorption capacity than the anterior ones, 
even in the UV-A range. The absorption coefficient values of the capsules are comparable with that of the layers taken from the anterior cortex.

\section{Contribution of the epithelium to the capsular UV absorbance}

The absorbance of the anterior capsule is determined by the capsule and the epithelial layer together. To determine the absorbance of the epithelium, the absorbances of separate anterior capsules with or without epithelium were measured. The two capsules possessed identical properties, e.g. age and thickness, see Figure 5 for further details. Figure 5A shows that the measured values differ only at wavelengths shorter than $300 \mathrm{~nm}$. From the difference of the two values, the absorbance and the absorption coefficient of the epithelium were determined. The absorbance values suggest that the epithelium does not contribute significantly to the total absorbance of the capsule, probably due to its limited thickness. On the other hand, the calculated absorption coefficient indicate a considerable absorption capacity in the UV-C range (Figure 5B).
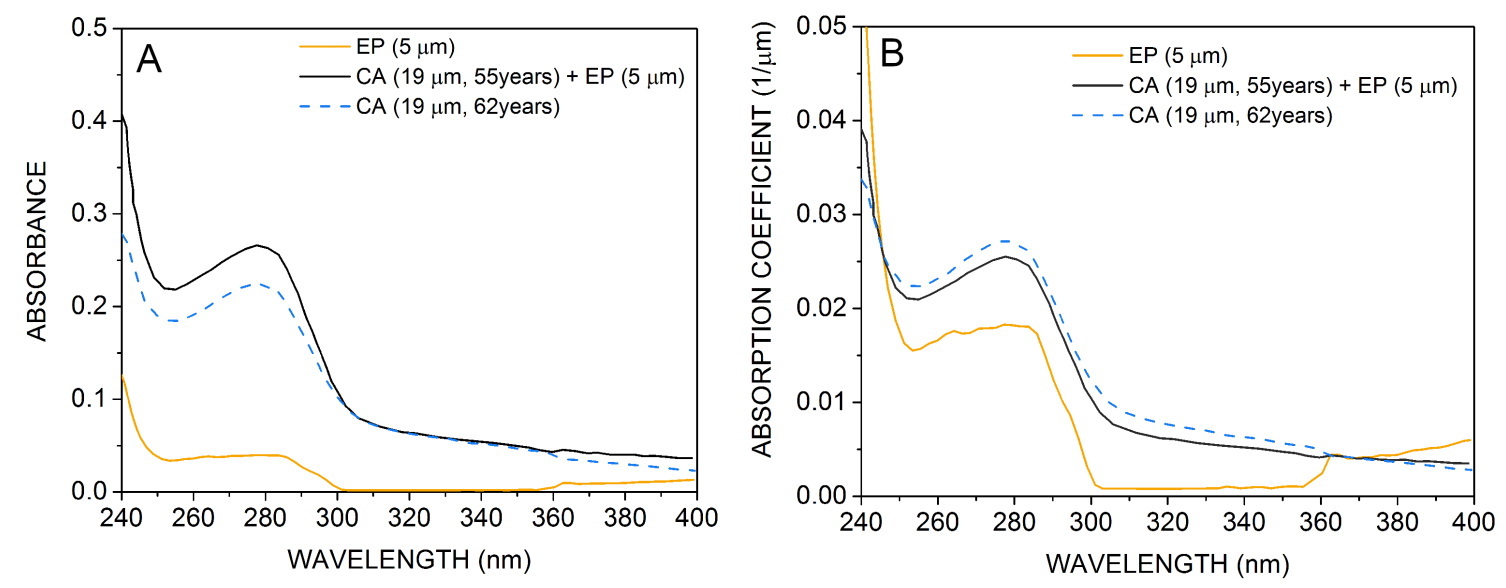

Figure 5. A: Absorbances of separate anterior capsules (CA) with (55 years old) and without (62 years old) epithelium (EP). The difference of the two values proposes the absorbance of the epithelium. B: Absorption coefficients as function of wavelength calculated from the measured absorbances.

\section{Transmission of the lens}

The results concerning the UV absorption of the lens were so far taken from the entire lens or lens homogenates, except the studies of Gaillard and of Dillon (Dillon et al., 1999; Gaillard et al., 2000). To determine the transmission of the entire lens, the cumulative 
absorbance was calculated using the averaged absorption curves in the 50-59 year age group. The absorption values of the discarded segments were assumed to be equal to the absorbance of the samples taken in front of the unused sections. The measured absorbance resulted from the absorption and scattering processes together. Although, the samples were placed between quartz plates in a drop of isotonic saline, the amount of light scattering through the lens sections is presumably still higher than in the case of the measurements related to the whole lens.
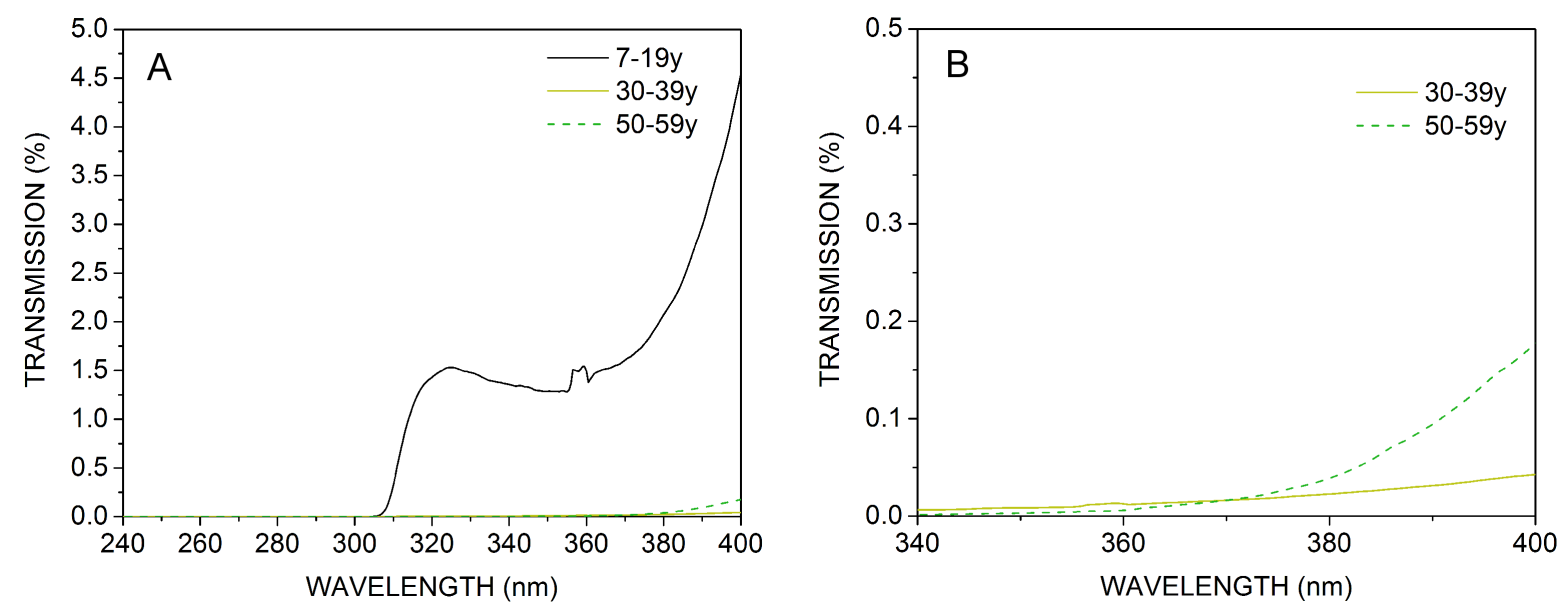

Figure 6. A: Calculated transmission of the lens as function of wavelength, in three age groups. $B$ : Calculated transmission of the two older groups in the $U V$-A range.

A relatively small difference in the scattering process can considerably affect the calculated absorbance due to the large number of lens sections ( $\mathrm{n}=60-70$ with $60 \mu \mathrm{m}$ thickness). In order to take this process into account, $2 \%$ of the incoming radiation was considered as scattered light in each step and the absorbance was reduced accordingly. The transmission was calculated by the combination of equations (2) and (3) and by using the corrected cumulative absorbance. In the case of the capsules, no corrections were made and simply the transmission was calculated from the averaged measured absorbance values.

Figure $6 \mathrm{~A}$ shows that only the youngest lenses transmit about $1.5-2 \%$ of incoming light in the UV-A range, while older lenses absorb almost the entire UV radiation (Figure 6B). It is also visible that the 7-19 years old age group shows a fine peak at wavelength of 320 $\mathrm{nm}$ which is related to the local minimum of the absorbance spectrum. These results are in good agreement with the data found in the literature. 
In the case of the capsules, both anterior and posterior capsules can absorb significant amount of radiation in the UV-C range only (Figure 7). Anterior capsules transmit about $10 \%$ less UV radiation than posterior ones, probably due to their increased thickness.
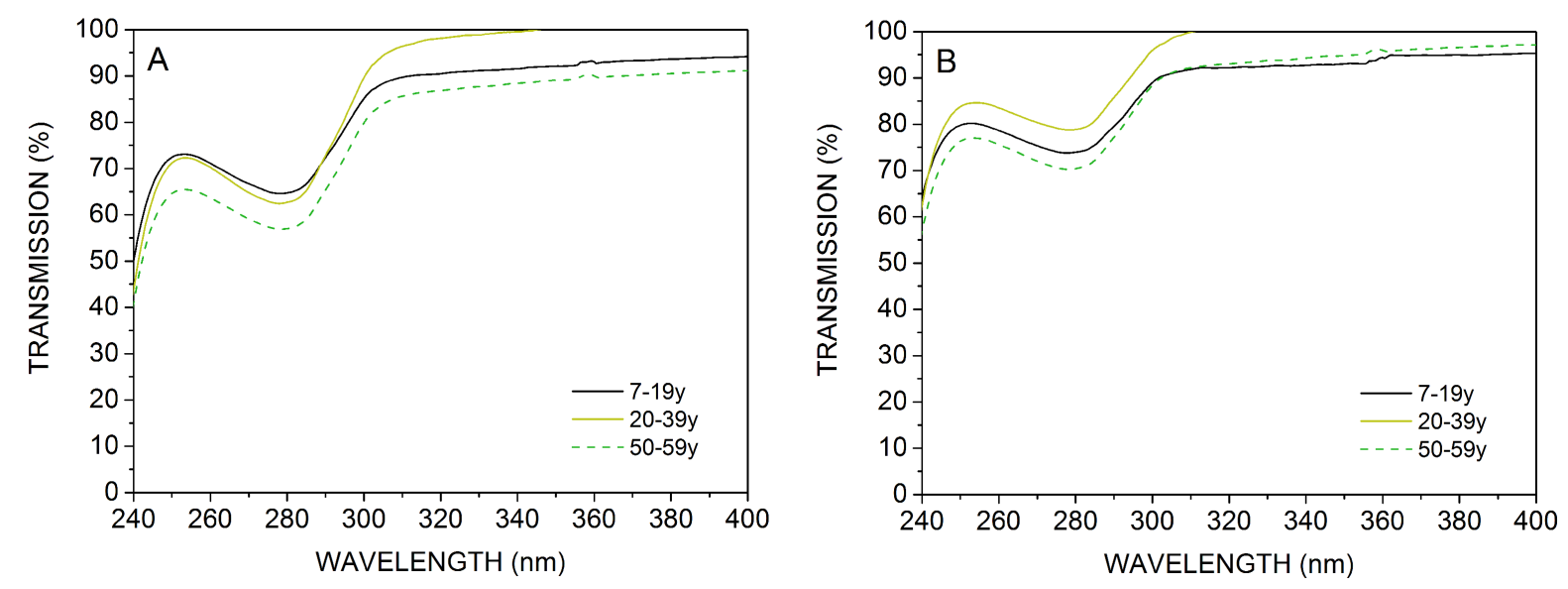

Figure 7. Calculated transmission of the anterior (A) and posterior (B) capsules as function of wavelength.

\section{Age-related changes of the UV absorption}

Figure 2 shows that the older lenses have higher absorption coefficients than the younger ones. Although, radiation with wavelengths shorter than $300 \mathrm{~nm}$ is presumably absorbed

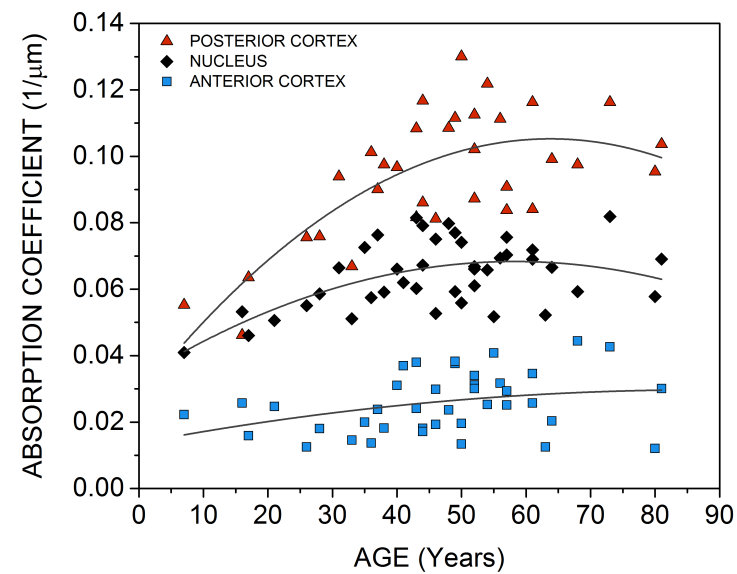

Figure 8. Averaged absorption coefficient taken at $280 \mathrm{~nm}$ as function of age. Solid lines show second order polynomial fit. terior and posterior cortex and the nucleus were calculated in the following way: average where the absorption spectrum has a maximum, offer the most plausible way to visualize more accurately the age-related changes of the UV-absorption of the lens. On the other hand, as the cornea is not able to fully absorb the UV radiation longer than $320 \mathrm{~nm}$, it is physiologically also relevant to investigate the age-related changes in the UV-A range.

The averaged absorption coefficients both at $280 \mathrm{~nm}$ and at $360 \mathrm{~nm}$ of the an-

by the cornea, the $280 \mathrm{~nm}$ wavelength, 
of the values of samples 1 and 2 provided the absorption coefficient of the anterior cortex, that of the nucleus was calculated from the data of layers 3-7, and the average of the spectra of samples 8 and 9 resulted in the absorption spectra of the posterior cortex. These calculations were performed for every single lens. The averaged values of the absorption coefficients at $280 \mathrm{~nm}$ as function of age are shown in Figure 8. It can be seen that the absorption coefficient of the posterior cortex is the highest and the nucleus and the anterior cortex have less efficient UV absorption capacity, independently of age. The second order polynomial fit clearly shows that the age-related changes of the posterior cortex are the most pronounced compared to the other two parts. The absorption coefficient increases more rapidly with age than that of the nucleus and reaches a plateau around the age of 60. Anterior cortex shows very moderate changes.
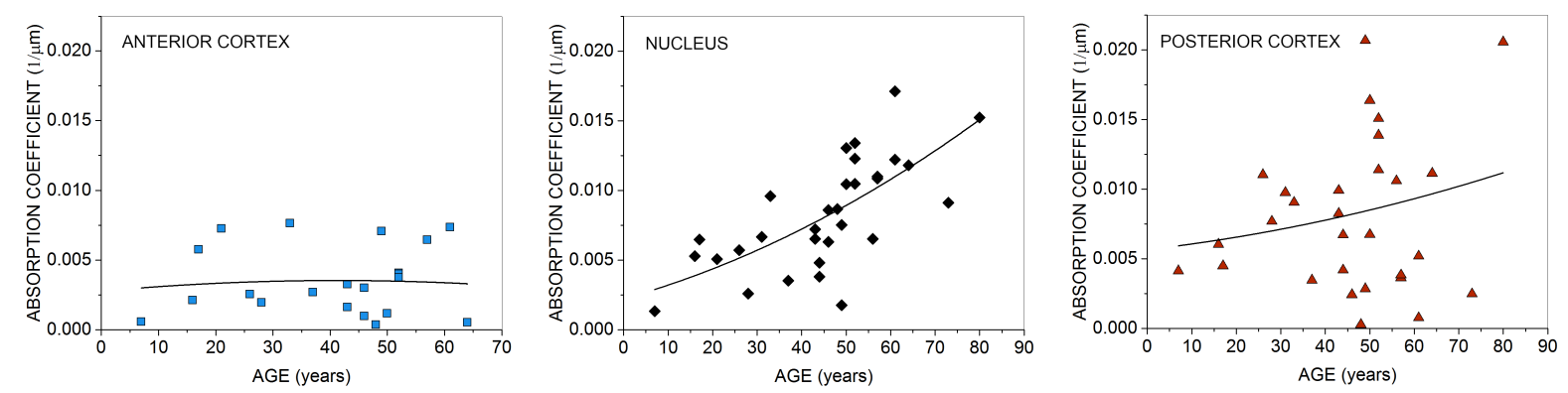

Figure 9. Averaged absorption coefficient taken at $360 \mathrm{~nm}$ as function of age. Solid lines show second order polynomial fit.

Averaged absorption coefficients taken at $360 \mathrm{~nm}$ are presented in Figure 9. Both the nucleus and the posterior cortex have increasing absorption capacity with age but changes of the nucleus are the most noticeable. The anterior cortex shows no age-dependent changes.

To determine exactly how the age affects the absorption properties of the different layers and whether this effect is significant, statistical analysis was performed. The age was considered as a continuous value and no age groups were formed. Values of the absorption coefficients taken at $280 \mathrm{~nm}$ of the nine sample layers of every single lens were collected. The ANCOVA model revealed a borderline significant interaction between age and layer $(\mathrm{p}=0.057)$. This result is significant at $10 \%$ level, and with respect to the moderate sample size this suggests that the effect of age depends on the layer. Changes 
of coefficient values as function of age were examined for each layer. Table 1 shows the correlation coefficients between age and the absorption coefficients at $280 \mathrm{~nm}$ and the results of significance tests for each layer. The correlation was significant in layers 6 ( $\mathrm{R}$ $=0.371, \mathrm{p}=0.022), 7(\mathrm{R}=0.49, \mathrm{p}=0.002)$ and $9(\mathrm{R}=0.363, \mathrm{p}=0.041)$. Indeed, the absorption coefficients of these layers demonstrate a moderate increasing tendency with age which can be seen on the slope of the linear regression (Figure 10).

\begin{tabular}{cccccccccc}
\hline Sample layers & 1 & 2 & 3 & 4 & 5 & 6 & 7 & 8 & 9 \\
\hline $\mathrm{R}$ & 0.056 & 0.160 & 0.158 & 0.136 & 0.215 & $\mathbf{0 . 3 7 1}$ & $\mathbf{0 . 4 9 0}$ & 0.096 & $\mathbf{0 . 3 6 3}$ \\
\hline p-value & 0.737 & 0.337 & 0.343 & 0.414 & 0.207 & $\mathbf{0 . 0 2 2}$ & $\mathbf{0 . 0 0 2}$ & 0.582 & $\mathbf{0 . 0 4 1}$ \\
\hline
\end{tabular}

Table 1. Test p-values of the ANOVA analysis related to the absorption coefficients taken at 280 $n m$. Values less than 0.05 were considered to be statistically significant (bold).
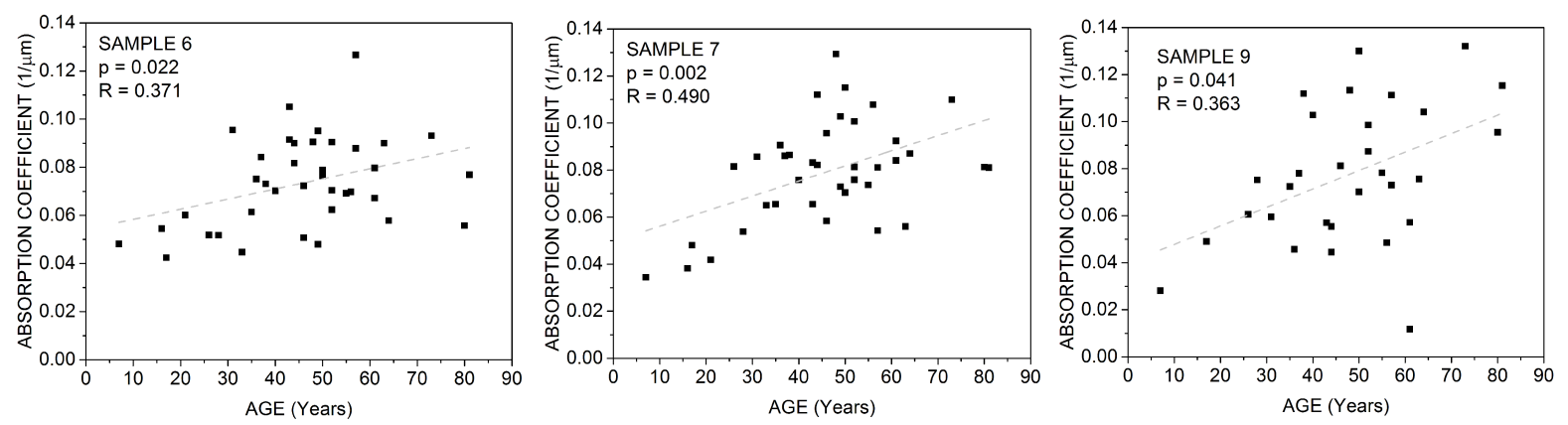

Figure 10. Absorption coefficients taken at $280 \mathrm{~nm}$ as function of age. The values are related to the age-dependent sample layers. Significant $R$ and $p$ values are indicated. Dashed line is the result of linear regression.

In a similar way, statistical analysis was performed in the case of absorption coefficients taken at $360 \mathrm{~nm}$. The ANCOVA model revealed a significant interaction between age and layer $(\mathrm{p}<0.01)$, indicating that the effect of age depends on the layer position in the UVA range. The correlation coefficient values between age and the absorption coefficient are presented in Table 2 . The correlation proved to be significant in layers $5(\mathrm{R}=0.379, \mathrm{p}=$ 0.047), $6(\mathrm{R}=0.614, \mathrm{p}=0.000)$, and $7(\mathrm{R}=0.676, \mathrm{p}=0.000)$. Again, the linear regression clearly shows a marked age-dependent increase of the absorption of these nuclear layers (Figure 11). In the case of samples 6 and 7, it is also visible that the absorption coefficient 

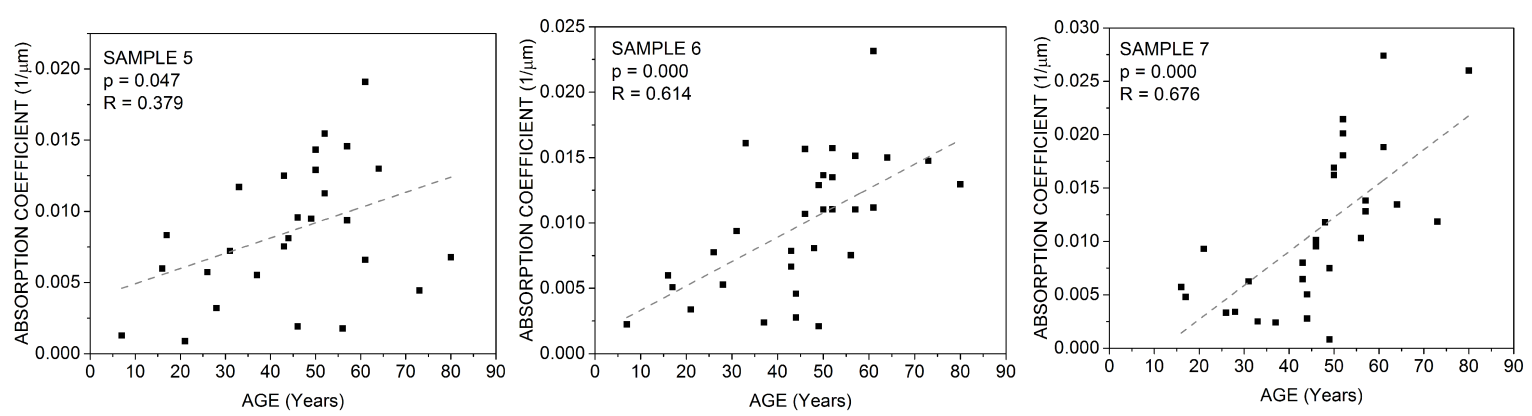

Figure 11. Absorption coefficients taken at $360 \mathrm{~nm}$ as function of age. The values are related to the age-dependent sample layers. Significant $R$ and $p$ values are indicated. Dashed line is the result of linear regression.

values are highly increased above the age of 50. From these data it can be concluded that in the near UV range, the absorption capacity of the posterior layers changes with age variably compared with the anterior ones.

\begin{tabular}{cccccccccc}
\hline Sample layers & 1 & 2 & 3 & 4 & 5 & 6 & 7 & 8 & 9 \\
\hline $\mathrm{R}$ & -0.160 & -0.320 & 0.129 & 0.337 & $\mathbf{0 . 3 7 9}$ & $\mathbf{0 . 6 1 4}$ & $\mathbf{0 . 6 7 6}$ & 0.195 & 0.261 \\
\hline p-value & 0.480 & 0.165 & 0.547 & 0.079 & $\mathbf{0 . 0 4 7}$ & $\mathbf{0 . 0 0 0}$ & $\mathbf{0 . 0 0 0}$ & 0.311 & 0.266 \\
\hline
\end{tabular}

Table 2. Test p-values of the ANOVA analysis related to the absorption coefficients taken at 360 $n m$. Values less than 0.05 were considered to be statistically significant (bold).

Values of the absorption coefficients taken at $280 \mathrm{~nm}$ of each capsule were collected and statistical analysis was performed in the similar way as in the case of the lens sections. Although the posterior capsules have higher averaged absorption coefficients than the anterior ones (Figure 4), results of ANCOVA showed that this difference is not significant $(\mathrm{p}=0.198)$. In addition, no correlation was found between the age and the absorption coefficients at $280 \mathrm{~nm}(\mathrm{p}=0.18)$. These findings indicate that the anterior and posterior capsules have almost equal absorption capacity and the age does not affect their optical properties.

\section{Protein expression in the anterior and posterior cortex}

It has been proven that there is an increasing absorption gradient along the optical axis independently of the anatomical structure (Figure 2). Interestingly, sections derived from 
the anterior and the posterior cortex have significantly different absorption capacity and only the posterior layers show age-dependent changes. As the optical properties of the lens are determined by the molecular components such as the crystallins, a gel-based proteomics study was carried out using lens sections taken from the anterior and posterior cortex in order to determine which proteins may be responsible for the anterior-posterior differences of UV absorption. Cryostat sections were cut from six lenses aged between 40 and 59 years as described earlier. The anterior lenticular pool of sections (group 1) consisted of sections 1 and 2, the posterior lenticular pool consisted of sections 8 and 9 (group 2). 2DE revealed a large series of spots $(\mathrm{n}=146)$ that can be seen in Figure 12. Altogether, nine proteins were found to produce significantly different levels between groups 1 (in A) and 2 (in B). These proteins are marked with their corresponding UniProtKB numbers. Several spots indicate the presence of identical proteins Beta-crystallin B1 and G3PD. To identify the proteins with significantly different expression levels, a MASCOT
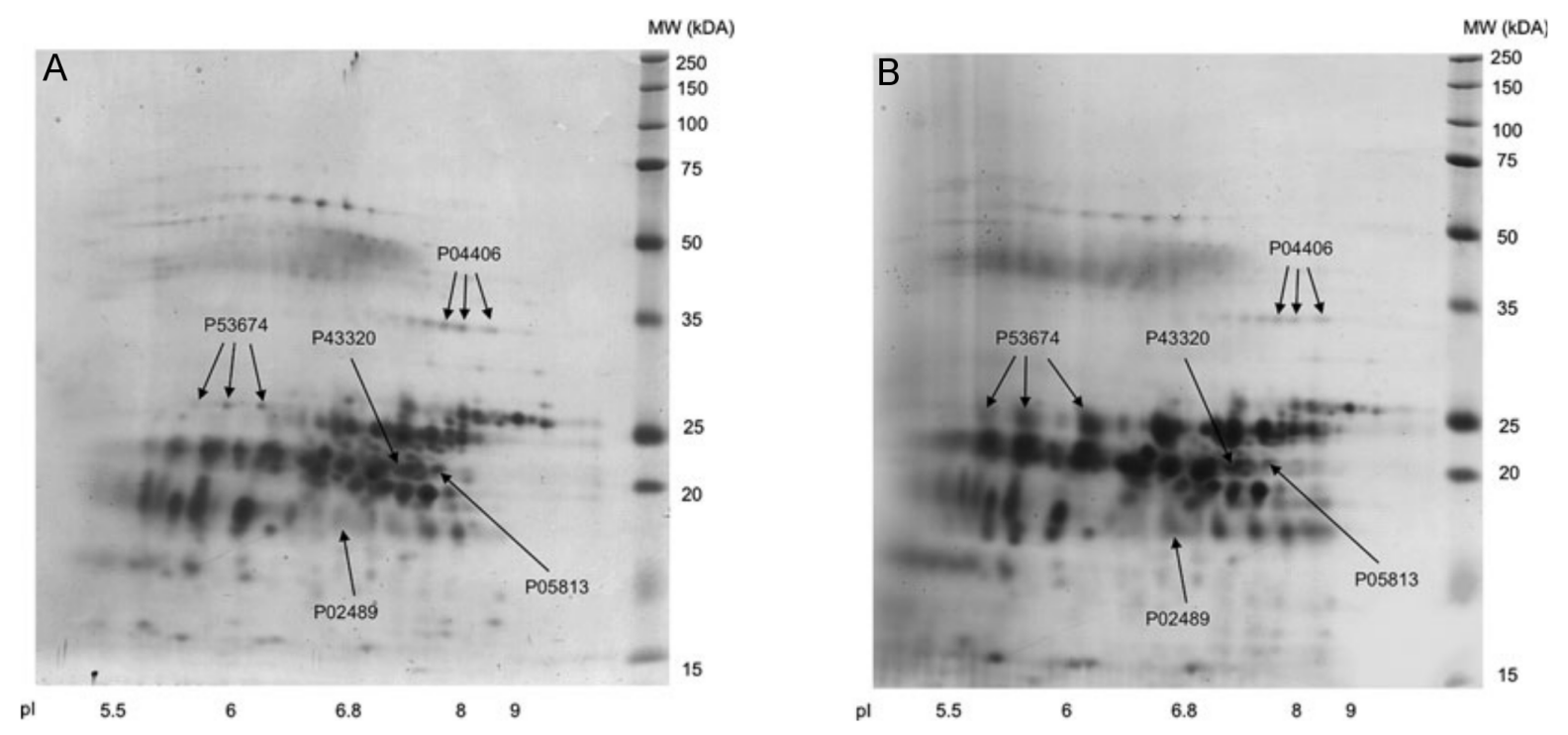

Figure 12. Master gels of group 1 (in A) and group 2 (in B) with the significantly different nine spot volumes from lens sections marked with UniProtKB accession numbers.

search was performed. The MASCOT software is one of the most universal algorithm for mass spectrum analysis and it can also predict or identify PTMs or other modifications such as amino acid conflicts. Complementary to the MASCOT search, Modiro TM search was also performed (ion scores/mass errors, sequence coverage, representative MS/MS spectra of Beta-crystallin B1 and G3PD and identified PTMs with a-, b-, y-, B-Pi, and 
y-Pi ion series can be found in the supplementary material of original paper I. (Pajer et $a l, 2013))$. Altogether, five individual proteins were identified with high significance and ion scores (Table 3).

Spots with significantly different level

\begin{tabular}{|ccccccc|}
\hline Protein & Accession no.* & \multicolumn{2}{c}{ Group 1 } & \multicolumn{4}{c}{ Group 2 } \\
mean & SD & mean & SD & P-value \\
\hline $\begin{array}{c}\text { G3P HUMAN } \\
\text { glyceraldehyd-3-phosphate } \\
\text { dehydrogenase }\end{array}$ & P04406 & 0.299 & 0.115 & 0.089 & 0.057 & 0.005 \\
\hline $\begin{array}{c}\text { CRYAA_HUMAN } \\
\text { Alpha-crystallin A chain }\end{array}$ & P02489 & 0.423 & 0.173 & 1.078 & 0.364 & 0.003 \\
\hline $\begin{array}{c}\text { CRBB1_HUMAN } \\
\text { Beta-crystallin B1 }\end{array}$ & P53674 & 0.594 & 0.239 & 1.522 & 0.503 & 0.002 \\
\hline $\begin{array}{c}\text { CRBB1_HUMAN } \\
\text { Beta-crystallin B1 }\end{array}$ & P53674 & 0.472 & 0.381 & 1.607 & 0.592 & 0.003 \\
\hline $\begin{array}{c}\text { CRBB1_HUMAN } \\
\text { Beta-crystallin B1 }\end{array}$ & P53674 & 0.197 & 0.182 & 1.104 & 0.373 & 0.001 \\
\hline $\begin{array}{c}\text { CRBB2_HUMAN } \\
\text { Beta-crystallin B2 }\end{array}$ & P43320 & 1.079 & 0.241 & 0.462 & 0.167 & 0.000 \\
\hline $\begin{array}{c}\text { CRBA1_HUMAN } \\
\text { Beta-crystallin A3 }\end{array}$ & P05813 & 1.095 & 0.343 & 0.472 & 0.104 & 0.004 \\
\hline
\end{tabular}

Verification of expression levels by in-gel ninhydrin assay

\begin{tabular}{|cccccccc|}
\hline Protein & Accession no. & \multicolumn{2}{c}{ Group 1 } & \multicolumn{4}{c}{ Group 2 } \\
mean & SD & mean & SD & Palue \\
\hline $\begin{array}{c}\text { G3P HUMAN } \\
\text { glyceraldehyd-3-phosphate } \\
\text { dehydrogenase }\end{array}$ & P04406 & 0.065 & 0.015 & 0.021 & 0.008 & 0.007 \\
\hline $\begin{array}{c}\text { CRYAA_HUMAN } \\
\text { Alpha-crystallin A chain }\end{array}$ & P02489 & 0.096 & 0.019 & 0.211 & 0.027 & 0.001 \\
\hline $\begin{array}{c}\text { CRBB1_HUMAN } \\
\text { Beta-crystallin B1 }\end{array}$ & P53674 & 0.147 & 0.017 & 0.398 & 0.068 & 0.001 \\
\hline $\begin{array}{c}\text { CRBB1_HUMAN } \\
\text { Beta-crystallin B1 }\end{array}$ & P53674 & 0.111 & 0.009 & 0.397 & 0.015 & 0.001 \\
\hline $\begin{array}{c}\text { CRBB1_HUMAN } \\
\text { Beta-crystallin B1 }\end{array}$ & P53674 & 0.040 & 0.014 & 0.269 & 0.032 & 0.001 \\
\hline $\begin{array}{c}\text { CRBB2_HUMAN } \\
\text { Beta-crystallin B2 }\end{array}$ & P43320 & 0.242 & 0.027 & 0.101 & 0.021 & 0.001 \\
\hline $\begin{array}{c}\text { CRBA1_HUMAN } \\
\text { Beta-crystallin A3 }\end{array}$ & P05813 & 0.247 & 0.020 & 0.127 & 0.014 & 0.001 \\
\hline
\end{tabular}

Table 3. Proteins found to be present with significantly different levels in the lens. The quantification method is indicated above the related part of the table.

*UniProtKB ID: http://www.uniprot.org/uniprot/

High sequence coverage (max. $90.83 \%$ ) of these proteins was obtained from enzyme digestions, conditions and bioinformatic algorithms. All peptides were identified from 
trypsin, chymotrypsin digestion and from treatment with proteases widely used for mass spectrometric analysis. Verification of protein expression levels from each protein spot of 2DE gels was performed using in-gel ninhydrin assay. Table 3 shows the results of the two different quantification methods: spots with significantly different levels and the result of in-gel ninhydrin assay. Values of means, standard deviation (SD), and P-values are presented. It was found that Human G3PD (UniProtKB ID: P04406), Beta-crystallin B2 (UniProtKB ID: P43320), and Beta-crystallin A3 (UniProtKB ID: P05813) levels were higher in the anterior lenticular pool than in the posterior one. Levels of the Human Alpha-crystallin A chain (UniProtKB ID: P02489) and the Human Beta-crystallin B1 (UniProtKB ID: P53674) were higher in the posterior pool. It can be seen that the results of the two methods are in good agreement. These proteins may present the molecular basis for differences in the anterior and posterior UV absorbance. 


\section{Discussion}

\section{Posterior layers are more effective UV absorbers}

The absorbance of the different parts of the human lens and that of the capsules have been determined in the age range of 7 - to 81 years. The averaged absorption spectra showed that the lens sections can absorb significantly higher levels of radiation in the UV-B and -C ranges than in UV-A range with a maximum at wavelength of $280 \mathrm{~nm}$. Earlier studies presented that there is an additional peak at $360 \mathrm{~nm}$ with much lower values. Upon studying the absorption in the UV-A range, only fine absorption changes were observed in the case of young lenses.

Fiber cells of the ocular lens are arranged in a series of concentric shells, new growth shells are added continuously to the lens surface. Accordingly, the younger fibers compose the cortex and the nucleus consists of the older ones. Based on this structure, considerable difference in the absorption properties would be expected between the cortex and the nucleus only. Gaillard et al. (Gaillard et al., 2000) demonstrated that sections derived from the nucleus absorbed more UV radiation than slices taken from the outer cortex. These results are related only to the outer $1.0-1.25 \mathrm{~mm}$ part of the lens and thus there are no available data regarding the posterior cortex. The number of the samples was also very limited. In this study, altogether 38 human samples were used and the measurements were made along the full length of the optical axis which provides a more comprehensive view. Surprisingly, it was found that the UV absorption capacity increases anteroposteriorly inside the lens, independently of the anatomical structure. Despite the fact, that the posterior and anterior cortex consist of the same fibers, their absorption properties are very different. The obtained increasing trend is not explicit between the age of 7 and 19, the layers derived from the nucleus have almost equal absorption coefficients. This feature may be associated to the remodeling of the human lens which occurs at this age when the aspect ratio significantly changes and the primary fiber cell shortening happens (Augusteyn, 2010). In addition, the low-molecular-weight form of Alpha-crystallin is absent in the nucleus of the old lens in contrast to the peripheral part (Roy and Spector, 
1976). In young samples this protein is uniformly present throughout the lens and thought to be shifted to high-molecular-weight fractions such as Alpha-crystallin A. Beside these processes, the concentration of the UV filter molecules, such as $3 \mathrm{OHKG}$, is much higher in lenses younger than 20 years (Bova et al., 2001), which can also contribute to the different optical properties. At wavelengths longer than $310 \mathrm{~nm}$, the posterior layers are able to absorb higher amount of irradiation but the absorption gradient along the optical axis is not as definite as in the UV-C range. As our spectrophotometer was calibrated to detect the high absorbance values at $280 \mathrm{~nm}$, the relatively small changes in the UVA wavelength range was presumably detected with lower sensitivity. It is evident from the calculated transmission curves of the whole lens that the youngest age group has a fine peak at $320 \mathrm{~nm}$ while older lenses has almost zero transmission at wavelengths shorter than $400 \mathrm{~nm}$. These results are in good agreement with the work of Ambach et al. (Ambach et al., 1994).

The capsules can also effectively absorb the UV radiation and show very similar absorption spectrum as the lens sections. This is not surprising if we consider that both the capsule and the lens fibers are synthesized by the epithelial cells. The averaged absorption coefficients show that the posterior capsules have higher absorption capacity than the anterior ones but the difference is not statistically significant. Since the anterior capsule is much thicker than the posterior one, it can absorb higher amount of UV radiation in total. The epithelial layer has a comparable absorption coefficient to that of the rest of the anterior capsule but it does not contribute significantly to the absorbance due to its negligible thickness.

\section{Only the posterior layers show age-dependent changes}

It has been demonstrated that the absorption increases along the optical axis, independently of age. The maximum of the absorption spectra is higher in the case of the older lenses (Figure 2). The averaged absorption coefficients of the various parts of the lens indicate that at $280 \mathrm{~nm}$ wavelength, the age-related changes of the posterior cortex is the most noticeable while only moderate alterations can be observed in the case of the anterior cortex. Indeed, the statistical analysis proved that only posterior layers (6, 7 and 
9) show significant correlation with age. Several results suggest that there is a difference in protein expression levels along the visual axis. The water content in the posterior pole is lower than in the anterior one (Siebinga et al., 1990), indicating a higher protein concentration in the posterior pole. Experiments using different imaging techniques revealed spatial and age-dependent variations in crystallin content (Grey and Schey, 2009; Moffat and Pope, 2002) and the concentration of Alpha-crystallin also increases with age at the expense of Beta- and Gamma-crystallins (Bron et al., 2000; Satoh, 1972). In this study, it was found that levels of Alpha-crystallin A and Beta-crystallin B1 chains are higher in the posterior part. The crystallins have a similar absorption spectrum as the lens, thus they presumably strongly contribute to the UV absorption. The water soluble protein fraction decreases after middle age (Satoh, 1972; Zelentsova et al., 2017), resulting in decreased absorption at $280 \mathrm{~nm}$ wavelength. In accordance with these, the absorption coefficients of the posterior cortex and of the nucleus (Figure 8) display a plateau around the age of 60 years. The above results suggest that the age-related changes of the absorption at 280 nm are strongly related to the age-dependent protein expression levels.

Considering the UV-A range, the absorption gradient is not as definite as at the wavelength of $280 \mathrm{~nm}$ but it is still visible that the posterior part of the lens is more effective UV absorber. Figure 9 shows that the anterior cortex produces no age-related changes and at the same time, the averaged absorption coefficient of the oldest nuclear layers is three times higher than that of the younger ones. The statistical results confirmed that only the samples taken from the central and posterior nuclear region show correlation with age and indeed, the absorption coefficients of samples 6 and 7 are significantly higher at ages above 50. Mainly the UV-filter compounds, such as 3OHKG or 3OHKyn are responsible for the absorption in the UV-A and -B ranges. The levels of protein bound UV-filters in the nucleus is highly increased after middle-age, especially in the case of 3OHKG (Korlimbinis et al., 2007). On the other hand, the concentration of these Trp metabolites decreases with age (Bova et al., 2001), except the glutathione adduct of $3 \mathrm{OHKG}$, which is barely detectable in young lenses, but is present in a significantly high concentration in the aged nucleus. According to these results, the increased absorption in the posterior nuclear layers after middle age is possibly related to the age-dependent 
changes of $3 \mathrm{OHKG}$.

The absorption at the wavelength of $360 \mathrm{~nm}$ is increased in aged lenses. Earlier studies (Cooper and Robson, 1969; Gaillard et al., 2000) showed that the peak shifts towards the shorter wavelengths with age. It is visible that the youngest age group shows a maximum around $360 \mathrm{~nm}$ and the absorption is slightly decreased at $320 \mathrm{~nm}$ (Figure 3). This feature is not present in older age groups. Due to the calibration of the spectrophotometer, the absorption changes in the UV-A range is less pronounced than in the UV-C but the measured absorption spectra are in agreement with the data found in the literature.

The posterior capsules have slightly higher absorption capacity than the anterior ones independently of age, but significant age-related changes were not found. The major structural component of the capsule is Type IV collagen. Collagen molecules are extremely long-lived and as a consequence, post-translational modifications can occur to change the mechanical properties of the aged lens capsule (Danysh and Duncan, 2009; Krag and Andreassen, 2003; Krag et al., 1997). The lamellar structure of the anterior capsule also disappears with age. On the other hand, our findings indicate that the optical properties are not affected by these molecular changes. It is likely that the overall concentration of the components remains relatively constant and the structural modifications have no effect on it. However, an exact explanation would require further investigations.

These results suggest that the higher absorbance of the old lens is not only due to its greater thickness, but also to the increased absorption capacity of the posterior layers. Although the capsules show no age-related changes, the thicker anterior capsule also increases the total absorbance of the aging lens.

\section{Absorption gradient is accompanied by differential protein ex- pression levels}

Spectroscopic measurements indicate that there are spatial variations in crystallin content through the lens and noticeable differences were found between the protein levels in the nucleus and in the cortex (Moffat and Pope, 2002). In addition, the protein concentration is significantly higher at the posterior pole than at the anterior one (Siebinga et al., 1990). Alpha-crystallins also show spatial and age-related variations (Grey and Schey, 2009). In 
this work, it has been proved that the levels of Beta-crystallin B2, A3 and that of G3PD were significantly higher in the anterior part of the lens, meanwhile Alpha-crystallin A and Beta-crystallin B1 were present in higher concentration in the posterior cortex.

Alpha-crystallin is thought to be a major target for UV irradiation due to its higher degree of unfolding (Weinreb et al., 2000). Liang et al. (Liang et al., 1985a,b) have found that Trp residues of Alpha-crystallin are more exposed to UV radiation due to their greater degree of protein unfolding than Beta- or Gamma-crystallins. As Trp residues are mainly responsible for the absorbance peak at $280 \mathrm{~nm}$, Alpha-crystallin is suggested to play a major role in UV absorption compared with Beta- or Gamma-crystallins. Therefore, increased amounts of Alpha-crystallin in the posterior cortex may induce greater UV absorption than the anterior parts. Sites of attachment of Trp derived UV filters could be identified and linked to Beta-crystallin B1 (Aquilina and Truscott, 2002) and the difference between the anterior and posterior part in the overall protein concentration may also contribute to the increasing absorption gradient. Alpha-crystallin obtained from older lenses is more affected by UV irradiation than that taken from young lenses, especially in the case of Alpha-crystallin A chain, presumably because its unfolding increases with age. As a consequence, Alpha-crystallin may play a major role in the enhanced absorption of the posterior layers of the aged lenses. These findings may underlie the significant differences between the absorption properties of the anterior and posterior cortex but do not explain the gradient in the central part of the lens. The overall protein level in the nucleus is constant and much higher than in the cortex (Siebinga et al., 1990). Li has reported $(\mathrm{Li}, 1974)$ that the nuclear Alpha-crystallin shows higher absorbance than the cortical one, which may contribute to the enhanced absorption capacity of the posterior nuclear layers. On the other hand, the distribution of the different types of proteins in the nuclear region still remains an open question and it shall require further investigations to explain why the posterior cortex has even higher absorption capacity than the nucleus.

In rat lenses the level of G3PD was found to be significantly higher in the cortex than in the nucleus (Dovrat et al., 1984). In humans, the enzyme activity in the nucleus is lower than in the cortex (Yan et al., 2006), which may reflect a differential enzyme level. Here, it has been demonstrated that G3PD is present in higher concentration in the 
anterior cortex. As G3PD plays an important role in the glycolysis pathway, its increased level in the anterior section pool may be due to and reflects differences in carbohydrate metabolism within the lens. It has been shown that the concentration of G3PD in the whole lens is much lower above the age of 60 than between 40 and 55 years. In cataractous lenses the enzyme activity is also strongly reduced (Rachdan et al., 2004). Interestingly, G3PD was described as a target of UV-B (Schmidt et al., 1992) and old animal lenses are more susceptible to UV-induced cataract (Zhang et al., 2012).

In the view of the above results, it is clear that the differential UV-absorption of the lens is accompanied by differential protein expression levels. The age-related changes in absorption properties of the posterior parts may also be related to the age-dependent molecular changes. It remains an open question whether differential crystallin localization underlies or reflects different optical or biological functions of the lens. 


\section{Conclusions}

Here, we have demonstrated that the absorption of the human eye lens in the near-UV range increases antero-posteriorly and independently of the anatomical structure. Older lenses have higher absorption coefficients than the younger ones but only the posterior layers showed significant age-dependent changes. The enhanced absorption capacity of the posterior parts strongly contribute to the increased absorbance of the aged eye lens. Considering the UV-A range, the age has an effect on the posterior nuclear layers only. This is probably associated to different levels of the Trp-derived UV-filter compounds. The capsules are also effective UV absorbers and highly contribute to the total absorbance, especially in the UV-B and -C ranges and it seems that the age has no effect on their absorption capacity. It has been proved that the absorption gradient is accompanied by differential protein expression levels. The differential UV absorbance and its agedependent changes are strongly related to the various crystallin concentration.

These findings may help better understand the UV-induced cataract formation and could add considerable information to the design of intraocular lenses. Data related to the absorption of lens capsules could be also important in cataract surgery. 


\section{Acknowledgements}

I am indebted to Prof. Antal Nógrádi (Head of Laboratory of Neural Regeneration at the Department of Anatomy, Histology and Embryology, Faculty of Medicine, University of Szeged) for his professional mentorship and scientific guidance for my research.

I would like to express my gratitude to Prof. Béla Hopp (Head of Department of Optics and Quantumelectronics, Faculty of Science and Informatics, University of Szeged) for his professional help in the spectrophotometric measurements and guidance in the physics related aspects of the research.

Special thanks to my co-authors, especially to Prof. Gert Lubec and Ákos Tiboldi, for performing the two-dimensional gel electrophoresis at the Department of Pediatrics, Medical University of Vienna. I would like to thank Ferenc Rárosi for computing the statistical analysis at the Department of Medical Physics and Informatics, University of Szeged.

I am indebted to all members of the Laboratory of Neural Regeneration, especially to my brother, Krisztián Pajer for his support and help.

Finally, I would like to thank my beloved wife, Dóra Pajer-Boross who always supported me in my work with never ending enthusiasm. 


\section{References}

JL Alió, A Anania, and P Sagnelli. The aging of the human lens in Age-related changes of the human eye (ed. Cavallotti CAP and Cerulli L). Humana Press, Totowa, NJ, USA, 2008.

W Ambach, M Blumthaler, T Schöpf, E Ambach, F Katzgraber, F Daxecker, and A Daxer. Spectral transmission of the optical media of the human eye with respect to keratitis and cataract formation. Doc Ophthalmol, 88:165-173, 1994.

UP Andley and BA Clark. Generation of oxidants in the near-UV photooxidation of human lens alpha-crystallin. Invest Ophthalmol Vis Sci, 30:706-713, 1989.

JA Aquilina and RJ Truscott. Identifying sites of attachment of UV filters to proteins in older human lenses. Biochim Biophys Acta, 1596:6-15, 2002.

Age-Related Eye Disease Study Research Group: AREDS. Risk factors associated with age-related nuclear and cortical cataract: a case-control study in the age-related eye disease study, AREDS report no. 5. Ophthalmology, 108:1400-1408, 2001.

JM Artigas, A Felipe, A Navea, A Fandino, and C Artigas. Spectral transmission of the human crystalline lens in adult and elderly persons: color and total transmission of visible light. Invest Ophthalmol Vis Sci, 53:4076-4084, 2012.

RC Augusteyn. On the growth and internal structure of the human lens. Exp Eye Res, 90:643-654, 2010.

H Bloemendal. Molecular and cellular biology of the eye lens. John Wiley, New York, NJ, USA, 1981.

H Bloemendal, W de Jong, R Jaenicke, NH Lubsen, C Slingsby, and A Tardieu. Ageing and vision: structure, stability and function of lens crystallins. Prog Biophys Mol Bio, 86:407-485, 2004 .

TW Bochow, SK West, A Azar, B Munoz, A Sommer, and HR Taylor. Ultraviolet light exposure and risk of subcapsular cataracts. Arch Ophthalmol, 107:369-372, 1989.

EA Boettner and JR Wolter. Transmission of the ocular media. Invest Ophthalmol Vis Sci, 1:776-783, 1962.

LM Bova, AM Wood, JF Jamie, and RJW Truscott. UV filter compounds in human lenses: the origin of 4-(2-amino-3-hydroxyphenyl)-4-oxobutanoic acid O- $\beta$-D-glucoside. Invest Ophthalmol Vis Sci, 40:3237-3244, 1999.

LM Bova, MHJ Sweeney, JF Jamie, and RJW Truscott. Major changes in human ocular UV protection with age. Invest Ophthalmol Vis Sci, 42:200-205, 2001.

AJ Bron, GFJM Vrensen, J Koretz, G Maraini, and JJ Harding. The ageing lens. Ophthalmologica, 214:86-104, 2000. 
A Cetinel, V Semenchenko, JY Cho, MG Sharaf, KF Damji, LD Unsworth, and C Montemagno. UV-B induced fibrillization of crystallin protein mixtures. PLoS One, 12: e0177991, 2017.

WQ Chen, SU Kang, and G Lubec. Protein profiling by the combination of two independent mass spectrometry techniques. Nat Protoc, 1:1446-1452, 2006.

GF Cooper and JG Robson. The yellow colour of the lens of man and other primates. $J$ Physiol, 203:411-417, 1969.

CB Coulter, FM Stone, and EA Kabat. The structure of the ultraviolet absorption spectra of certain proteins and amino acids. J Gen Physiol, 19:739-752, 1936.

KJ Cruickshanks, BEK Klein, and R Klein. Ultraviolet light exposure and lens opacities: the Beaver Dam Eye Study. Am J Public Health, 82:1658-1661, 1992.

BP Danysh and MK Duncan. The lens capsule. Exp Eye Res, 88:151-164, 2009.

WW De Jong, JAM Leunissen, PJM Leenen, A Zweers, and M Versteeg. Dogfish- $\alpha$ crystallin sequences. Comparison with small heat shock proteins and schistosoma egg antigen. J Biol Chem, 263:5141-5149, 1988.

WF Diao, H Hoger, WQ Chen, A Pollack, and G Lubec. Estrotus-cycle-dependent hippocampal of signaling proteins. Hippocampus, 17:563-576, 2007.

J Dillon. The photophysics and photobiology of eye. J Photochem Photobiol B: Biol, 10: 23-40, 1991.

J Dillon, L Zheng, JC Merriam, and ER Gaillard. The optical properties of the anterior segment of the eye: implications for cortical cataract. Exp Eye Res, 68:785-795, 1999.

PJ Dolin. Ultraviolet radiation and cataract: a review of the epidemiological evidence. Brit J Ophthalmol, 78:478-482, 1994.

A Dovrat, J Scharf, and D Gershon. Glyceraldehyde 3-phosphate dehydrogenase activity in rat and human lenses and the fate of enzyme molecules in the aging lens. Mech Ageing Dev, 28:187-191, 1984.

N Fujii, H Uchida, , and T Saido. The damaging effect of UV-C irradiation on lens alpha-crystallin. Mol Vis, 10:814-820, 1999.

ER Gaillard, L Zheng, JC Merriam, and J Dillon. Age-related changes in the absorption characteristics of the primate lens. Invest Ophthalmol Vis Sci, 41:1454-1458, 2000.

E Ganea and JJ Harding. $\alpha$-crystallin assists the renaturation of glyceraldehyde-3phosphate dehydrogenase. Biochem J, 345:467-472, 2000.

AR Goldfarb, LJ Saidel, and E Mosovich. The ultraviolet absorption spectra of proteins. J Biol Chem, 193:397-404, 1951. 
H Gray. Gray's anatomy: The anatomical basis of clinical practice, 40th edition (ed. Susan Standring). Churchill Livingston, Elsevier, London, UK, 2008.

AC Grey and KL Schey. Age-related changes in the spatial distribution of human $\alpha$ crystallin products by MALDI imaging mass spectrometry. Invest Ophthalmol Vis Sci, 50:4319-4329, 2009.

PJTA Groenen, KB Merck, W de Jong, and H Bloemendal. Structure and modification of the junior chaperone alpha-crystallin. From lens transparency to molecular pathology. Eur J Biochem, 225:1-19, 1994.

ES Holiday. Spectrophotometry of proteins. I. Absorption spectra of tyrosin, tryptophan and their mixtures. II. Estimation of tyrosine and tryptophan in proteins. Biochem J, 30:1795-1803, 1936.

F Hollows and D Moran. Cataract - the ultraviolet risk factor. Lancet, 318:1249-1250, 1981.

BD Hood, B Garner, and RJW Truscott. Human lens coloration and aging. Evidence for crystallin modification by the major ultraviolet filter, 3-hydroxykynurenine O- $\beta$-Dglucoside. J Biol Chem, 274:32547-32550, 1999.

J Horwitz. Lens $\alpha$-crystallin: function and structure. Eye, 13:403-408, 1999.

J Horwitz. Alpha-crystallin. Exp Eye Res, 76:145-153, 2003.

SU Kang and G Lubec. Determination of in-gel protein concentration by a ninhydrinbased method. Proteomics, 11:481-484, 2011.

SU Kang, S Heo, and G Lubec. Mass spectrometric analysis of GABA-A receptor subtypes and phosphorylations from mouse hippocampus. Proteomics, 11:2171-2181, 2011.

L Kessel, L Eskilden, JH Lundeman, OB Jensen, and M Larsen. Optical effects of exposing intact human lenses to ultraviolet radiation and visible light. BMC Ophthalmol, 41:1-7, 2011.

BEK Klein, R Klein, and KE Lee. Incidence of age-related cataract: the Beaver Dam Eye Study. Arch Ophthalmol, 116:219-225, 1998.

L Kolozsvári, A Nógrádi, B Hopp, and Zs Bor. UV absorbance of the human cornea in the 240- to $400 \mathrm{~nm}$ range. Invest Ophthalmol Vis Sci, 43:2165-2168, 2002.

A Korlimbinis, JA Aquilina, and RJW Truscott. Protein-bound UV filters in normal human lenses: the concentration of bound UV filters equals that of free UV filters in the center of older lenses. Invest Ophthalmol Vis Sci, 48:1718-1723, 2007.

S Krag and TT Andreassen. Mechanical properties of the human posterior lens capsule. Invest Ophthalmol Vis Sci, 44:691-696, 2003. 
S Krag, T Olsen, and TT Andreassen. Biomechanical characteristics of the human anterior lens capsule in relation with age. Invest Ophthalmol Vis Sci, 38:357-363, 1997.

H Langen, P Berndt, D Roder, N Cairns, G Lubec, and M Fountoulakis. Two-dimensional map of human brain proteins. Electrophoresis, 20:907-916, 1999.

JS Lee, JH Liao, SH Wu, and SH Chiou. $\alpha$-crystallin acting as a molecular chaperonin against photodamage by UV irradiation. J Protein Chem, 16:283-289, 1997.

LK Li. Physical and chemical changes in alpha-crystallin during maturation of lens fibers. Exp Eye Res, 18:383-393, 1974.

JN Liang, UP Andley, and LT Chylack. Spectroscopic studies on human lens crystallins. Biochim Biophys Acta, 832:197-203, 1985a.

JN Liang, SK Bose, and B Chakrabarti. Age-related changes in protein conformation in bovine lens crystallins. Exp Eye Res, 40:461-469, 1985b.

S Löfgren. Solar ultraviolet radiation cataract. Exp Eye Res, 156:112-116, 2017.

J Mellerio. Yellowing of the human lens: nuclear and cortical contributions. Vision Res, 27:1581-1587, 1987.

JC Merriam. The concentration of light in the human lens. J Am Ophthalmol, 94:803-918, 1996.

LM Meyer, AR Wegener, FG Holz, M Kronschläger, JP Bergmanson, and PG Soderberg. Ultrastructure of UVR-B-induced cataract and repair visualized with electron microscopy. Acta Ophthalmol, 92:635-643, 2014.

BA Moffat and JM Pope. The interpretation of multi-exponential water proton transverse realaxation in the human and porcine eye lens. Magn Res Imag, 20:83-93, 2002.

Y Murata. Light absorption characteristics of the lens capsule. Ophthalmic Res, 19: 107-112, 1987.

RE Neale, JL Purdie, LW Hirst, and AC Green. Sun exposure as a risk factor for nuclear cataract. Epidemiology, 14:707-712, 2003.

A Pirie. Formation of N'-Formylkynurenine in proteins from lens and other sources by exposure to sunlight. Biochem J, 125:203-208, 1971.

DG Pitts, AP Cullen, and PD Hacker. Ocular effects of ultraviolet radiation from 295 to $365 \mathrm{~nm}$. Invest Ophthalmol Vis Sci, 16:932-939, 1977.

D Rachdan, MF Lou, and JJ Harding. Revival of inactive glyceraldehyde 3-phosphate dehydrogenase in human cataract lenses by reduction. Exp Eye Res, 79:105-109, 2004.

JE Roberts. Ocular phototoxicity. Photochem Photobiol B, 64:136-143, 2001. 
AM Rosen, DB Denham, V Fernandez, D Borja, A Ho, F Manns, JP Parel, and RC Augusteyn. In vitro curvatures and dimensions of human lens. Vision Res, 46:1002-1009, 2006.

$\mathrm{K}$ Rosenheck and P Doty. The far ultraviolet absorption spectra of polypeptide and protein solutions and their dependence on conformation. Biochemistry, 47:1775-1785, 1961.

D Roy and A Spector. Absence of low-molecular-weight alpha crystallin in nuclear region of old human lenses. Proc Nat Acad Sci USA, 73:3484-3487, 1976.

K Satoh. Age-related changes in the structural proteins of human lens. Exp Eye Res, 14: 53-57, 1972.

J Schmidt, C Schmitt, M Kojima, and O Hockwin. Biochemical and morphological changes in rat lenses after long-term UV-B irradiation. Ophthalmic Res, 24:317-325, 1992.

I Siebinga, GFJM Vrensen, FFM De Mul, and J Geve. Age-related changes in the local water protein content of human eye lenses measured by Raman microspectroscopy. Exp Eye Res, 53:233-239, 1990.

C Slingsby and NJ Clout. Structure of the crystallins. Eye, 13:395-402, 1999.

C Slingsby, GJ Wistow, and AR Clark. Evolution of crystallins for a role in the vertebrate eye lens. Protein Sci, 22:367-380, 2013.

PG Söderberg, PO Rol, DB Denham, and JM Parel. Transmittance of the lens capsule. Proceedings of SPIE - The International Society for Optical Engineering, 1996.

HR Taylor. The environment and the lens. Brit J Ophthalmol, 64:303-310, 1980.

HR Taylor, SK West, FS Rosenthal, B Munoz, HS Newland, H Abbey, and EA Emmett. Effect of ultraviolet radiation on cataract formation. N Eng J Med, 22:1429-1433, 1988.

VL Taylor, AJ Al-Ghoul, CW Lane, JR Davis, and MJ Costello. Morphology of the normal human lens. Invest Ophthalmol Vis Sci, 37:1396-1410, 1996.

RJW Truscott, AM Wood, JA Carver, MM Sheil, GM Stutchbury, J Zhu, and GW Kilby. A new UV-filter compound in human lenses. FEBS Letters, 348:173-176, 1994.

S Vazquez, A Aquilina, JF Jamie, MM Sheil, and RJW Truscott. Novel protein modification by kynurenine in human lenses. J Biol Chem, 277:4867-4873, 2002.

SSS Wang and WS Wen. Examining the influence of ultraviolet C irradiation on recombinant human $\gamma$ D-crystallin. Mol Vis, 16:2777-2790, 2010.

LA Weale. Age and transmittance of the human crystalline lens. J Physiol, 395:577-587, 1988. 
O Weinreb, MAM van Boekel, A Dovrat, and H Bloemendal. Effect of UV-A light on the chaperone-like properties of young and old lens $\alpha$-crystallin. Invest Ophthalmol Vis Sci, 41:191-198, 2000.

SK West, DD Duncan, B Munoz, GS Rubin, LP Fried, K Bandeen-Roche, and OD Schein. Sunlight exposure and risk of lens opacities in a population-based study. JAMA, 280: 714-718, 1998.

C Winding, Y Sun, H Höger, H Bubna-Littitz, A Pollak, P Schmindt, and G Lubec. Serine/threnonine-protein phosphatase $1 \alpha$ levels are paralleling olfactory memory formation in the CD1 mouse. Electrophoresis, 32:1675-1683, 2011.

AM Wood and RJW Truscott. UV filters in human lenses: tryptophan catabolism. Exp Eye Res, 56:317-325, 1993.

AM Wood and RJW Truscott. Ultraviolet filter compounds in human lenses: 3-hydroxykynurenine glucoside formation. Vision Res, 34:1369-1374, 1994.

H Yan, MF Lou, MR Fernando, and JJ Harding. Thioredoxin, thioredoxin reductase, and $\alpha$-crystallin revive inactivated glyceraldehyde 3-phosphate dehydrogenase in human aged and cataract lens extracts. Mol Vis, 12:1153-1159, 2006.

EA Zelentsova, LV Yanshole, AZ Fursova, and YP Tsentalovich. Optical properties of the human lens constituents. J Photoch Photobio B, 173:318-324, 2017.

J Zhang, H Yan, S Löfgren, X Tian, and MF Lou. Ultraviolet radiation-induced cataract in mice: the effect of age and the potential biochemical mechanism. Invest Ophthalmol Vis Sci, 53:7276-7285, 2012.

M Zhu, J Yu, Q Gao, J Wang, L Hu, Y Zheng, F Wang, and Y Liu. The relationship between disability-adjusted life years of cataracts and ambient erythemal ultraviolet in China. J Epidemiol, 25:57-65, 2015.

S Zigman. Ocular light damage. Photochem Photobiol, 57:1060-1068, 1993.

S Zigman. Environmental near-UV radiation and cataracts. Optometry Vision Sci, 72: 899-901, 1995.

S Zigman, T Paxhia, T McDaniel, MF Lou, and NT You. Effect of chronic near-ultraviolet radiation on the gray squirell lens In Vivo. Invest Ophthalmol Vis Sci, 32:1723-1732, 1991. 
Appendix 


$$
\text { I. }
$$




\title{
The Molecular Background of the Differential UV Absorbance of the Human Lens in the $240-400 \mathrm{~nm}$ Range
}

\author{
Viktor Pajer ${ }^{\dagger 1,2}$, Ákos Tiboldi ${ }^{\dagger 3}$, Narkhyun $\mathrm{Bae}^{3}$, Kongzhao $\mathrm{Li}^{3}$, Sung Ung $\mathrm{Kang}^{3}$, Béla Hopp ${ }^{4}$, \\ Lajos Kolozsvári ${ }^{1}$, Gert Lubec ${ }^{3}$ and Antal Nógrádi ${ }^{\star 1}$ \\ ${ }^{1}$ Department of Ophthalmology, Albert Szent-Györgyi Clinical Centre, Faculty of Medicine, University of Szeged, Hungary \\ ${ }^{2}$ Department of Optics and Quantum Electronics, University of Szeged, Hungary \\ ${ }^{3}$ Department of Pediatrics, Medical University of Vienna, Vienna, Austria \\ ${ }^{4}$ Research Group on Laser Physics, Hungarian Academy of Sciences and University of Szeged, Hungary \\ Received 7 November 2012, accepted 7 February 2013, DOI: 10.1111/php.12063
}

\begin{abstract}
The ultraviolet (UV) absorption of various sections of the human lens was studied and compared with protein expression paralleling differential UV absorbance in anterior and posterior lenticular tissue. The UV absorbance of serial lens cryostat sections $(60 \mu \mathrm{m})$ and that of lens capsules was determined using a Shimadzu scanning spectrophotometer, and the absorption coefficients were calculated. Two-dimensional gel electrophoresis was performed using two pooled lenticular protein extracts (anterior and posterior sections). Protein spots were quantified and significantly different spots were identified by mass spectrometry following in-gel digestion with trypsin and chymotrypsin. The UV-C and UV-B absorption of the human lens increased toward the posterior parts of the lens. The anterior and posterior lens capsules also effectively absorbed UV radiation. Levels of molecular chaperone proteins Beta-crystallin B2 (UniProtKB ID:P43320), A3 (UniProtKB ID:P05813) and of glyceraldehyde 3-phosphate dehydrogenase (UniProtKB ID:P04406) were significantly higher in the anterior part of the lens, whereas lens proteins Beta-crystallin B1 (UniProtKB ID:P53674) and Alpha-crystallin A chain (UniProtKB ID:P02489) were higher in the posterior sections. These results provide evidence that differential UV absorption in the anterior and posterior lens is accompanied by differential protein expression.
\end{abstract}

\section{INTRODUCTION}

Ultraviolet (UV) radiation poses an increasing risk on the tissues and organs exposed to radiation. In the human eye, the cornea absorbs most of the UV radiation (1), but still significant radiation may reach the lens. It is well established that UV radiation, especially within the range of UV-B $(280-330 \mathrm{~nm})$, may cause ocular damage (2,3). Exposure to near UV light (315-400 nm) has been reported to lead to pathological changes in the lens of albino mice and gray squirrels $(4,5)$. Several studies provided evidence for the relationship between the risk of cataract and sun

$\uparrow$ These authors have equally contributed to this study

§Current address: Department of Anatomy, Histology and Embryology H-6724 Szeged Kossuth L. Sgt. 40. Hungary

*Corresponding author email: nogradi@opht.szote.u-szeged.hu (Antal Nógrádi)

(C) 2013 Wiley Periodicals, Inc.

Photochemistry and Photobiology (C) 2013 The American Society of Photobiology 0031-8655/13 exposure (2,6-9). Brown type of human cataracts develops much more frequently in outdoor-active individuals than in indoor workers (9) and an increased risk of posterior subcapsular cataract is associated with high exposure to UV-B radiation (6).

The primate lens absorbs most of the UV-A (330-400 nm) and UV-B (280-330 nm) radiation (10). Accordingly, it is thought that under normal circumstances no considerable amounts of UV radiation reach the retina. Therefore, it is of critical importance to determine to what degree various sections of the lens and its capsule contribute to the capacity of the lens to absorb UV radiation. An earlier study has shown that the various layers of the cornea are able to absorb UV radiation to different extent (1). There are only few studies concerning the absorption properties of the different layers of the lens. Gaillard et al. (11) found, that in primates, the posterior layers of the lens show higher UV absorbance than the anterior ones in the 300-440 nm range. Another study by Dillon et al. (12) showed that the nucleus of an old human lens reveals higher absorbance than the outer cortex. Moreover, no UV absorption data are available on the lens capsules.

The most abundant known low-molecular-weight UV-filter compounds in the human lens are kynurenine, 3-hydroxykynurenine glucoside (3OHKG), and 4-(2-amino-3-hydroxyphenyl)-4oxobutanoic acid O-glucoside (13). The UV spectra of these molecules show higher absorbance in the UV-B and UV-C ranges than in the UV-A range. Results suggest that these tryptophan-derived UV filters are responsible for UV radiation absorbance in the lens. Levels of these UV filters and protein-bound kynurenine and 3-hydroxykynurenine (3OHKyn) are higher in the nucleus than in the cortex (14). Accordingly, the nuclear concentration of free UV filters $30 H K G$ and $30 H K y n$ has been shown to be lower than that in the cortex (15). Apart from these low-molecular-weight compounds proteins may significantly contribute to UV absorption capacity.

The human lens has the highest protein content within the body and all proteins are absorbing in the UV range (16). Key proteins, i.e., crystallins of the lens have earlier been described in detail as major constituents and recently identified by mass spectrometry, too (17). These specific lenticular proteins are main determinants for optical properties including UV absorbance. To the best of our knowledge, however, the presence of these proteins was not localized to anterior or posterior parts of the lens. 
It was therefore the aim of the current study to determine the UV absorption of various parts of the lens and the lens capsules and to carry out a gel-based proteomic study of human anterior and posterior lenticular parts. And indeed, anterior and posterior sections showed differential protein expressional patterns that were paralleling differential UV absorbance. Our findings on differential crystallin expressions in the anterior and posterior sections are in agreement with previous work on the role of lenticular proteins for UV absorption $(18,19)$.

\section{MATERIALS AND METHODS}

Cryostat sectioning. Human lenses $(n=25)$ were obtained from eyes with a post-mortem delay of $6 \mathrm{~h}$. Most of the available lenses were between the age of 40 and 60 years and this age group was split in two: lenses taken from 40-49 $(n=10)$ and from 50-59 $(n=9)$ years old eyes were used for UV-absorption measurements. The rest of the lenses $(n=6)$ were used for proteomic studies.

The human lenses were used with the approval of the Human Ethics Committee of University of Szeged, Albert Szent-Györgyi Clinical Centre and the study was conducted in accordance with the provisions of Declaration of Helsinki for experimentation involving human tissue. The eyeballs were transported and stored in a humid chamber at $4^{\circ} \mathrm{C}$ until the lenses were removed. Lenses were stored at $-70^{\circ} \mathrm{C}$ until used and did not reveal any cataract formation. To determine the absorbance of the various sections of the lens, $60 \mu \mathrm{m}$ thick consecutive cryostat sections were cut at $-18^{\circ} \mathrm{C}$. The day before sectioning, the lenses were placed into cold $30 \%$ sucrose in PBS and stored at $+4^{\circ} \mathrm{C}$. The absorbance of $30 \%$ sucrose solution was determined $(60 \mu \mathrm{m}$ thick layer) and was found negligible. Before sectioning, the anterior and posterior lens capsules were removed and stored in physiological saline separately. During cryostat sectioning, the anterior $200 \mu \mathrm{m}$ thick portion of the lens was discarded, so the first section was taken from $200 \mu \mathrm{m}$ anteroposteriorly (Fig. 1). The next two sections were not used and the second sample was taken posterior to these. Accordingly, we had two $60 \mu \mathrm{m}$ thick samples from the anterior cortex. Posterior to that, every seventh section was kept for measurement. Thus, five samples were taken from the nucleus, and two samples from the posterior cortex with a $120 \mu \mathrm{m}$ thick discarded portion between them. Altogether, nine sections were taken for UV measurements in an anteroposterior order. Figure 1 shows the position of the sections in the lens and Table 1 summarizes the calculated anteroposterior depth of sections used for UV absorbance measurements. To avoid light scattering, the lens samples and the capsules were placed between two quartz glass plates with a drop of isotonic saline on both sides of the specimen. A $60 \mu \mathrm{m}$ thick spacing ring was placed between the plates to prevent compression damage to the samples.

UV absorbance spectrophotometric measurements. UV absorbance of samples was measured by a scanning spectrophotometer (model UV2101PC UV-VIS; Shimadzu, Kyoto, Japan). This model contains two spectral lamps (halogen lamp for 900-340 nm, and deuterium lamp for 340-190 nm wavelength ranges), a double monochromator to select the proper wavelengths, measuring and referencing beam paths, and a photomultiplier to detect and amplify the measuring and reference light intensities. On the reference side, only a drop of isotonic saline was placed between the quartz plates. The spectrophotometer was connected to a computer and the absorption spectrum was analyzed using the software of the spectrophotometer. The absorbance of the central portions of the lens sections was determined using an aperture with $2 \mathrm{~mm}$ diameter thus only the representative part of lens was measured (anterior and posterior cortex and nucleus), no matter, whether the peripheral parts of the section involved other parts of the lens (e.g. cortex in case of sections of the nucleus, See Fig. 1 for details). The absorbance and the absorption coefficient of the samples were calculated as in our earlier report (1). The investigated wavelength range was $200-400 \mathrm{~nm}$. The absorbance measurement range of our device was between 0 and 5 as the recorded absorbance values were often found to be greater than 4 at wavelengths below $240 \mathrm{~nm}$. Therefore, the accuracy of our measurements in the studied wide measuring range did not make possible to detect minute changes of absorbance as observed by Gaillard et al. around $360 \mathrm{~nm}$ wavelength (11). As the recorded results often approached or reached the upper instrument rating, therefore we present the absorbance data only in 240 $400 \mathrm{~nm}$ range in our calculations.

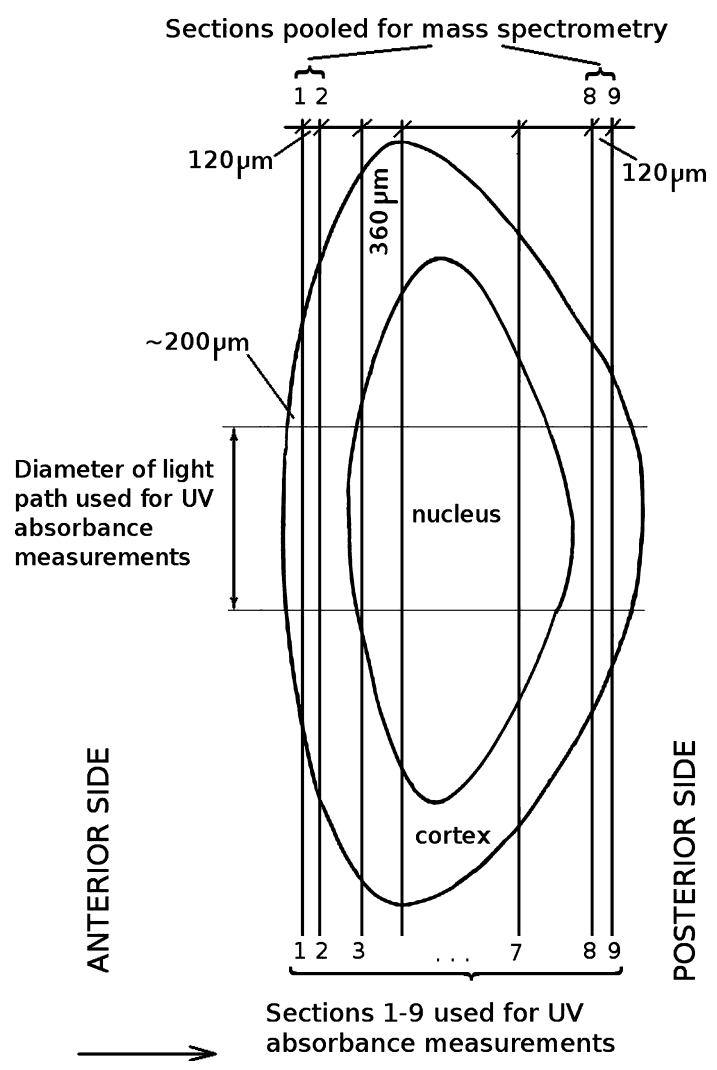

Figure 1. The position of the different sample layers within the lens. The first two samples $(1,2)$ were taken from the anterior cortex, the next five $(3-7)$ from the nucleus and the last two samples $(8,9)$ derived from the posterior cortex. Layers 1 and 2 as well as 8 and 9 were used for proteomics studies.

Table 1. The calculated anteroposterior position of the sections processed for UV measurement within the lens. Average thickness of the whole lens was approximately $3.95 \mathrm{~mm}$.

\begin{tabular}{llllllllll}
\hline Sample & 1 & 2 & 3 & 4 & 5 & 6 & 7 & 8 & 9 \\
\hline
\end{tabular}

$\begin{array}{llllllllll}\text { Anteroposterior } & 0.20 & 0.38 & 0.80 & 1.22 & 1.64 & 2.06 & 2.48 & 3.56 & 3.74\end{array}$ depth (mm)

Following the absorbance measurements, cryostat cross sections were cut from the anterior and posterior capsules. Absorbance was determined after trypsin treatment only as the repeated measurement procedure destroyed the thin samples. Therefore, anterior capsules with the same characteristics (age and thickness) were used as positive controls.

Proteomic studies. Two-Dimensional Gel Electrophoresis (2DE). Cryostat sections were cut from six lenses as described above and the anterior lenticular pool of sections consisted of sections 1 and 2, the posterior lenticular pool consisted of sections 8 and 9 . These pools were stored at $-80^{\circ} \mathrm{C}$ until gel-based proteomics studies. 2DE was performed essentially as reported previously (20-22).

Ouantification of protein levels. Excess dye from 2DE gels were removed by washing with distilled water and gels were scanned with an Image Scanner (Amersham Pharmacia Biotech). Protein spots from each gel (six gels per group, $n=12$ ) were outlined (first automatically and then manually) and quantified using the ProteomweaverTM software (Bio-Rad, CA, USA). The percentage of the volume of the spots representing a certain protein was determined in comparison with the total proteins present in the $2 \mathrm{DE}$ gel (23)

Analysis of peptides by Nano-LC-ESI-MS/MS (High-capacity ion trap, $H C T$ ). Fourteen spots which showed different levels between group 1 (anterior pool) and group 2 (posterior pool) were manually excised and placed into $1.5 \mathrm{~mL}$ Protein LoBind Eppendorf tubes. In-gel digestion and sample preparation for HCT analysis was performed as described before (23). The extracted peptides were pooled for Nano-LC-ESI-CID/ETD-MS/MS 

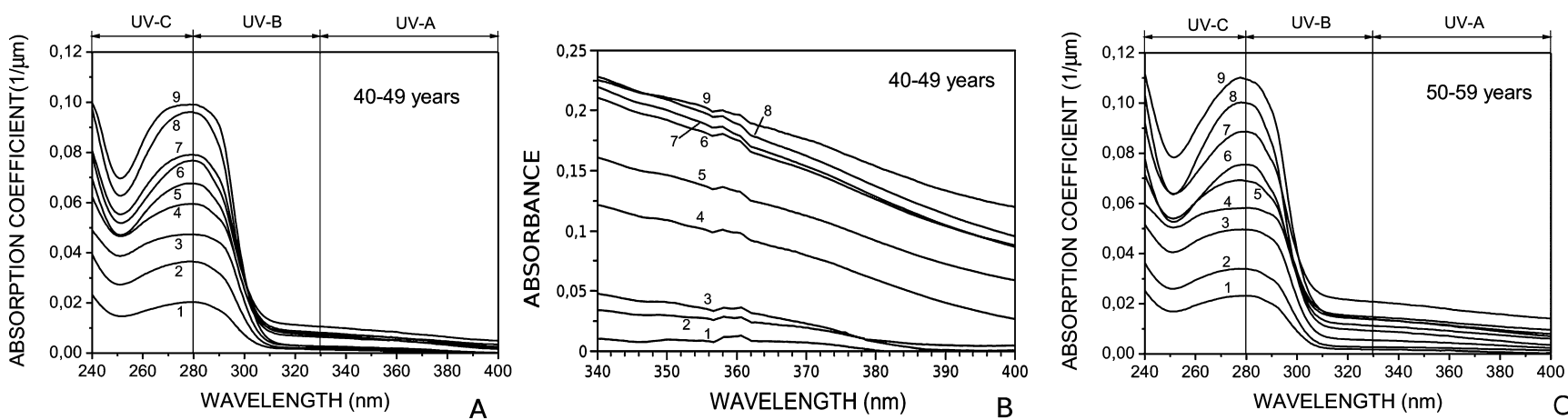

Figure 2. (A) Averaged absorption coefficients of the nine investigated layers as a function of wavelength in the age group of 40-49 years $(n=10)$ (B) Enlarged view of the curves shown in A in the 340-400 nm wavelength range. Note the fine elevated UV absorption at $360 \mathrm{~nm}$. (C) Averaged absorption coefficients of the nine investigated layers as a function of wavelength in the age group of 50-59 years $(n=9)$.

analysis as described in a previous article [24]. Protein identification and modification information returned from MASCOT were manually inspected and filtered to obtain confirmed protein identification and modification lists of CID MS/MS and ETD MS/ MS. Posttranslational modification searches were done using ModiroTM v1.1 software (Protagen AG, Germany). Protein identification and modification information returned were manually inspected and filtered to obtain confirmed protein identification and modification lists. (24).

Verification of protein expression levels as evaluated by Proteomeweaver $^{T M}$ by in-gel ninhydrin assay. Each protein spot was cut out and put into a $0.5 \mathrm{~mL}$ Protein LoBind tube (Eppendorf, Hamburg, Germany). Spots were initially washed twice with ACN: de-ionized water $(1: 1, \mathrm{v} / \mathrm{v})$ for $20 \mathrm{~min}$. Subsequently $100 \mu \mathrm{L}$ of $100 \%$ ACN was added to each tube to cover the gel piece completely and incubated for at least $2 \mathrm{~min}$. After the removal of ACN, gel pieces were dried completely in a SpeedVac Concentrator 5301. The dried gel pieces were reswollen with $60 \mu \mathrm{L}$ of $2 \mathrm{ng} / \mu \mathrm{L}$ proteinase K (Promega, Madison, WI, USA) buffered in $1 \mathrm{mM}$ $\mathrm{HCl}$. Gel pieces were incubated for longer than $4 \mathrm{~h}$ at $37^{\circ} \mathrm{C}$. Supernatants were transferred to new $0.5 \mathrm{~mL}$ tubes, and gel pieces were extracted by $80 \mu \mathrm{L}$ of $1 \%$ formic acid (Fluka, Steinheim, Germany) under sonication for $30 \mathrm{~min}$. Following this extraction step, peptides were eluted from the gel pieces by $20 \%$ ACN under sonication for $30 \mathrm{~min}$ and subsequently by $40 \% \mathrm{ACN}$ for $30 \mathrm{~min}$. The individual extractions were pooled into the $0.5 \mathrm{~mL}$ LoBind tubes containing the supernatant including proteinase $\mathrm{K}$. The volume was reduced to a final volume $<10 \mu \mathrm{L}$ in a SpeedVac concentrator and undergoing acid hydrolysis and ninhydrin reaction. Ninhydrin reaction was carried out as follows: Samples were hydrolyzed in $0.5 \mathrm{~mL}$ Eppendorf Protein LoBind tubes with $150 \mu \mathrm{L}$ of $6 \mathrm{~N}$ hydrochloric acid solution (Fluka) at $99^{\circ} \mathrm{C}$ for $24 \mathrm{~h}$. Hydrolysates were spun down and evaporated in an SpeedVac concentrator (Eppendorf). The dried material was redissolved in $20 \mu \mathrm{L}$ of distilled water, vortexed and centrifuged. Ninhydrin working solution was prepared as referenced [25]: $200 \mathrm{mg}$ of ninhydrin reagent (Sigma Chemical Co., Steinheim, Germany) was dissolved in a mixture of $7.5 \mathrm{~mL}$ of ethylene glycol (Sigma Chemical Co.) and $2.5 \mathrm{~mL}$ of $4 \mathrm{M}$ acetate buffer and $250 \mu \mathrm{L}$ of the stannous chloride (Sigma Chemical Co.) solution (add $50 \mathrm{mg}$ of SnCl2 to $500 \mu \mathrm{L}$ of ethylene glycol) were added with stirring. The reagent should be pale red in color. A quantity of $100 \mu \mathrm{L}$ of the final mixture was transferred to a flat bottom NUNC MaxiSorp microtiter 96-well plate (Thermo scientific, Langenselbold, Germany) and the mixture was kept in an incubator (MELAG OHG Medizintechnik, Berlin) at $100^{\circ} \mathrm{C}$ for $20 \mathrm{~min}$. Absorption was measured on an iMark microplate reader (Bio-Rad) at $570 \mathrm{~nm}$. Measurement of all the samples was performed within $10 \mathrm{~min}$ (25).

Statistical analysis. Statistical analysis to reveal between-group differences was performed by paired Student's $t$-test. Bonferroni-Holm and LSD correction was applied for correction of multiple testing. In all proteomic studies, a probability level of $P<0.01$ was considered statistically significant. All calculations were performed using SPSS version 14.0 (SPSS Inc., Chicago, IL).

\section{RESULTS}

The highest absorption levels of the samples were detected in the UV-B and UV-C range. Figures $2 \mathrm{~A}-\mathrm{C}$ show the averaged absorption coefficients of 10 samples in the first, and nine samples in the second age group as function of wavelength in the range of 240 $400 \mathrm{~nm}$.

In the UV-A range, a slow increase of absorption coefficient can be seen, which continues in the UV-B range too. At $360 \mathrm{~nm}$, a mild and transient increase of absorption coefficient is observed, which can be best seen when the range of absorption coefficient is scaled only up to one unit (Fig. 2B). From about $310 \mathrm{~nm}$, the absorption coefficient steeply increases and reaches a maximum at $280 \mathrm{~nm}$ (end of UV-B range). From this wavelength to $250 \mathrm{~nm}$ the absorption coefficient decreases and then increases again. The samples, which were taken from more posterior parts of the lens, show the same characteristics, but the absorption coefficients of the posterior layers are higher. For example, at $280 \mathrm{~nm}$, the absorption coefficient of the eighth sample is approximately five times greater than that of the first one.

Absorption coefficients of the anterior $(n=14)$ and posterior $(n=13)$ lens capsules, and the average of these coefficients were calculated too (Fig. 3), as function of wavelength.

It appears that the absorption of the capsules shows the same characteristics as the various layers of the lens and absorption coefficient of the lens capsules have approximately the same

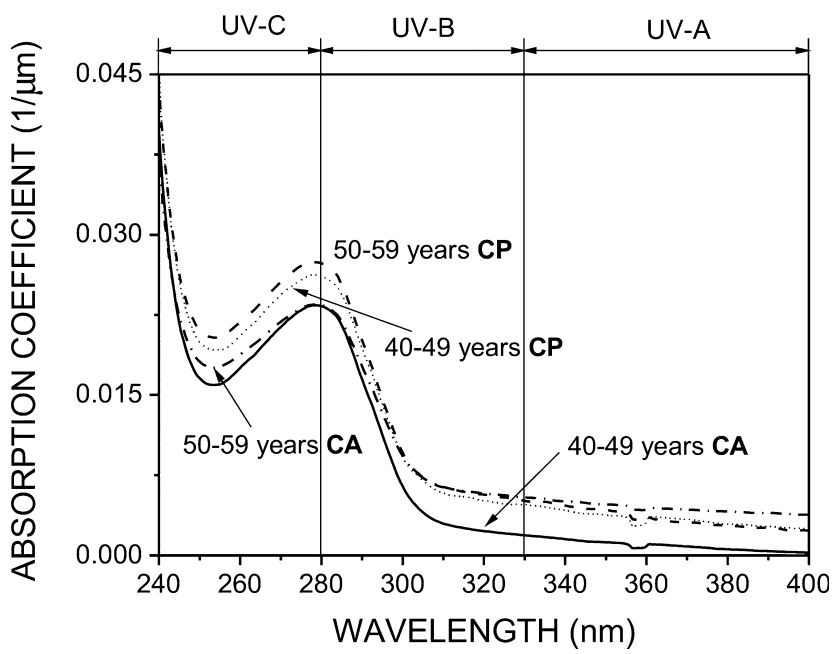

Figure 3. Averaged absorption coefficients of the anterior (CA) and posterior (CP) lens capsules as function of wavelength. Posterior capsules have higher absorption coefficients relative to anterior capsules at the wavelength of $280 \mathrm{~nm}$. Note the minor age difference in the averaged absorption coefficient values of the posterior capsules at wavelengths 250 $-280 \mathrm{~nm}$. 
value as the anteriormost layer of the lens (Fig. 2A and B). Absorption is more effective in UV-B and UV-C ranges than in UV-A range, with absorption maximum at $280 \mathrm{~nm}$. Moreover, it is evident that the posterior lens capsules have greater absorption coefficients than the anterior capsules in each age group but this difference was not significant. The thickness of the anterior lens capsule changes with age while that of the posterior capsule is approximately constant (26). This was confirmed by our measurements too. Thickness of the anterior lens capsules increased with age, from $20.92 \mu \mathrm{m}$ (age of 42 years) to $24.74 \mu \mathrm{m}$ (age of 57 years), while that of the posterior capsules remained relatively constant between 8.22 and $10 \mu \mathrm{m}$.

The absorbance of the anterior lens capsule is given together by the absorbance of the capsule and that of the epithelial lining. To determine the absorbance of the epithelium, the absorbances of separate lens capsules with or without epithelium (after trypsin treatment), but possessing identical properties (age and thickness) were measured. Then from the difference of the two values the absorbance and the absorption coefficient of the epithelium were determined. Figure 4 shows the absorbance of a 55 years old anterior lens capsule with epithelium and that of a 62 years old anterior capsule without epithelium. It can be seen that the two lines differ only at wavelengths below $300 \mathrm{~nm}$. The difference of the two values proposes the absorbance of the epithelium. It appears that the epithelium does not contribute considerably to absorbance.

To determine which proteins may underlie anterior-posterior differences of UV absorption, a gel-based proteomics study was carried out. 2D gel electrophoresis revealed a large series of spots $(n=146)$. A representative two-dimensional gel identifying proteins with differential levels in group 1 (samples 1 and 2) and group 2 (samples 8 and 9) along with their corresponding UniProtKB numbers is shown in Fig. 5.

Quantification results of nine proteins with significantly different levels between group 1 and 2 are listed in Table 2. Identifica-

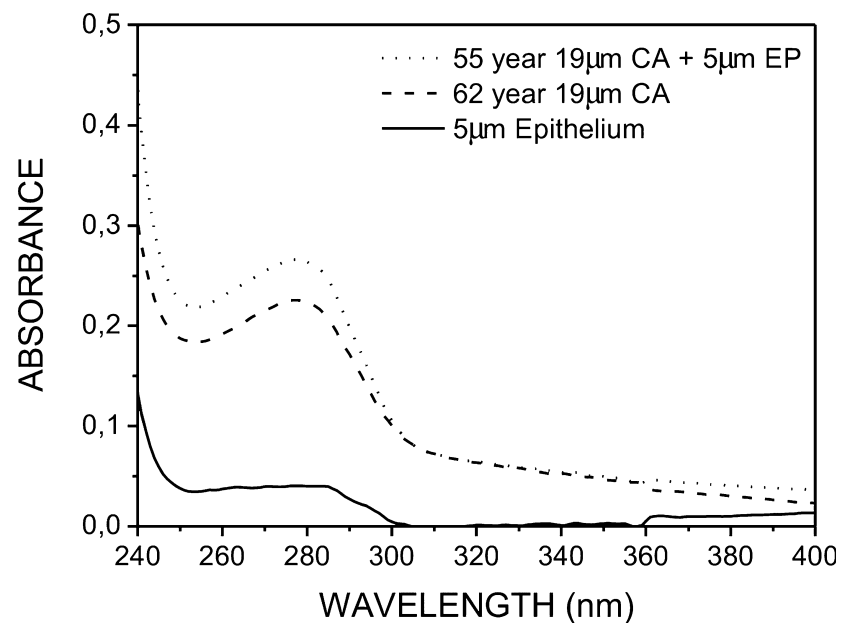

Figure 4. Absorbance of the anterior lens capsules with and without epithelium. The continuous line shows the calculated absorbance of the epithelium (EP). The anterior capsules (CA) had comparable physical properties.

tion of these nine protein spots along with their matched peptide numbers, sequence coverage and identification by Mascot software including ion scores/mass errors and MS/MS peptides determined are listed in Table S1. MASCOT software is one of the most universal algorithm for mass spectrum analysis and it can also predict or identify posttranslational modifications (PTMs) or other modifications such as amino acid conflicts. Complementary to the MASCOT search, Modiro ${ }^{\mathrm{TM}}$ search was also performed and as shown in Table S2 all five individual proteins were identified with high significance and ion scores. Table S3 shows the sequence coverage of all five individual proteins from different enzyme digestions, conditions, and different search algorithms. By combining results from different enzymes and bioinformatic algorithms, high sequence coverage (maximum
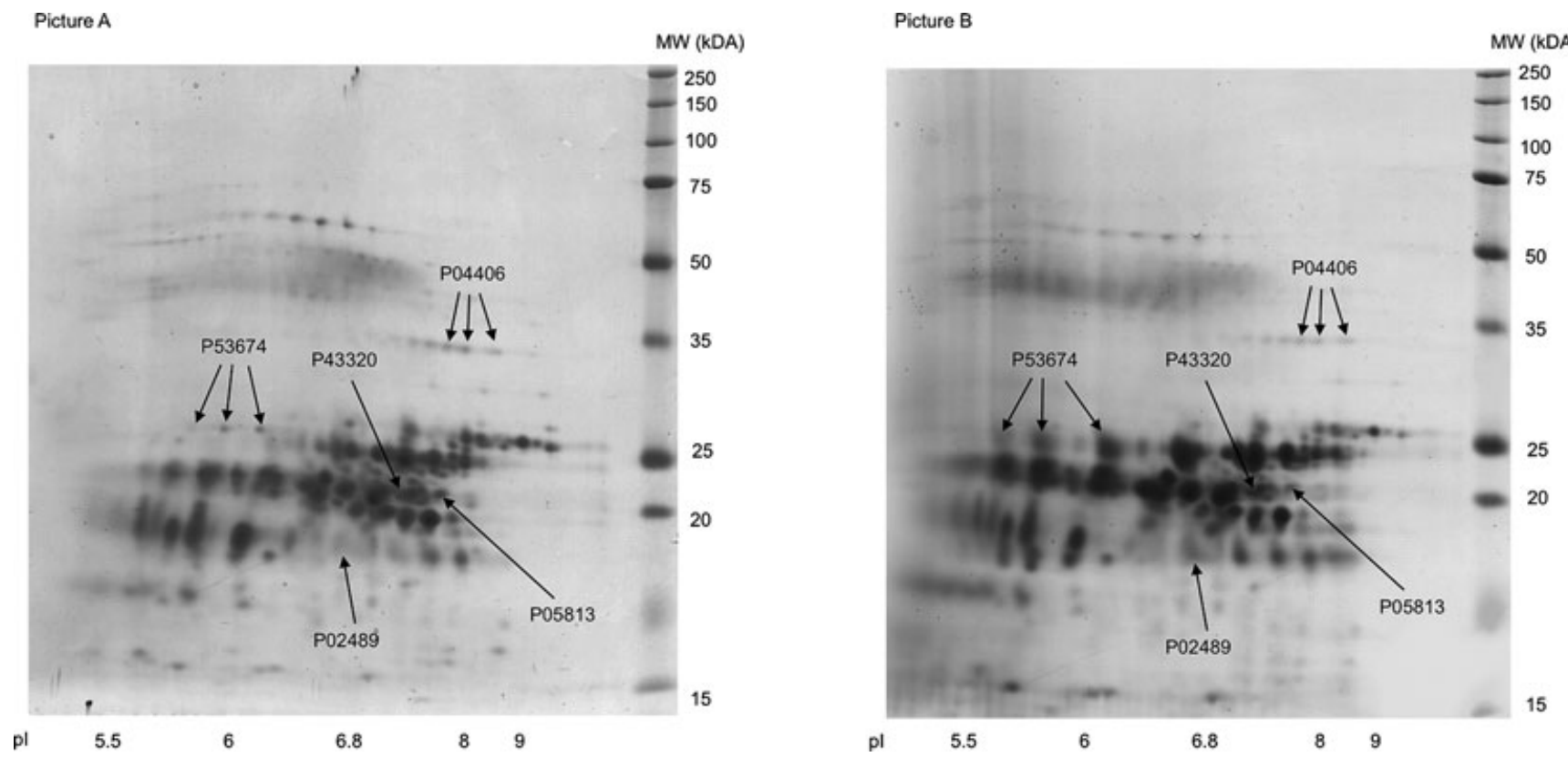

Figure 5. Picture A and B show the master gels of group A and group B, respectively, with the significantly changed 9 spot volumes from eye lens samples. UniProtKB accession numbers are provided. Several spots indicate the presence of identical proteins Beta-crystallin B1 and glyceraldehyde-3 phosphate dehydrogenase. 


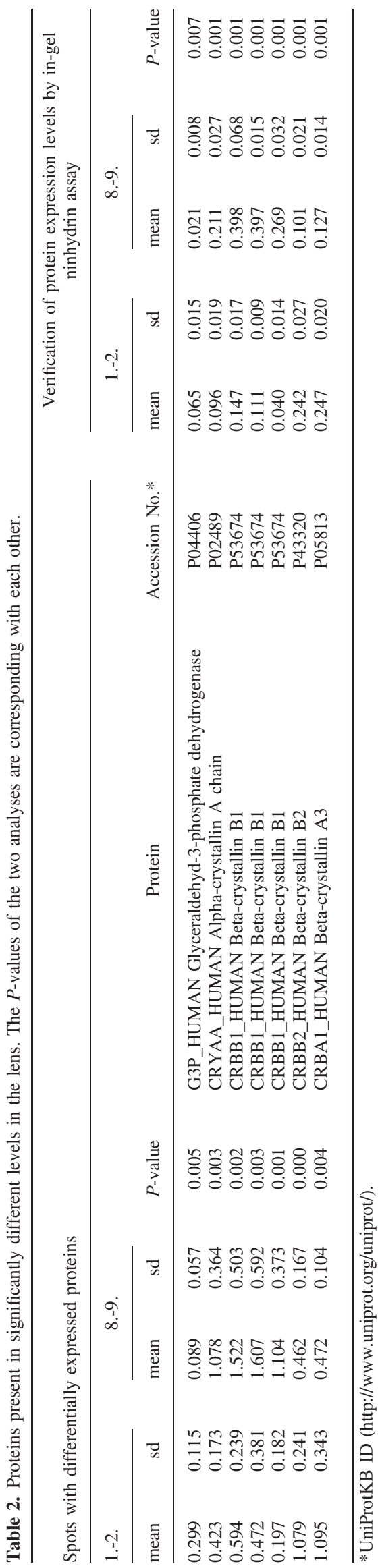

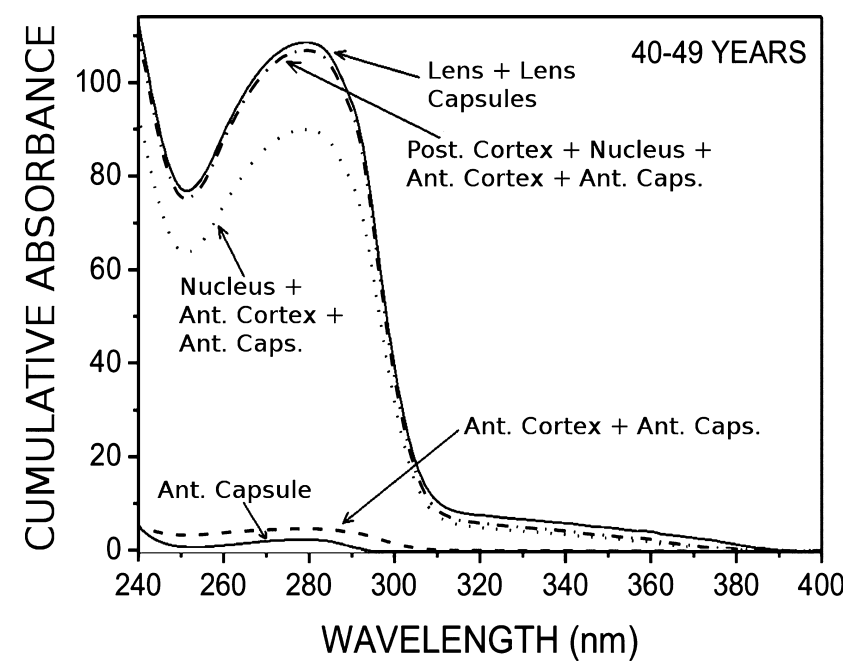

Figure 6. Cumulative absorbance of the whole lens. Absorbances of the various structural layers 1-9 were added together.

90.83\%) was obtained. All peptides were identified from trypsin, chymotrypsin digestion, proteases widely used for mass spectrometric analysis. Verification of protein expression levels from each protein spot of 2DE gels was performed using in-gel ninhydrin assay. Table 2 shows the results of the two different quantification methods providing means, $\mathrm{SD}$, and $P$-values. Human glyceraldehyde 3-phosphate dehydrogenase, Beta-crystallin B2, and Beta-crystallin A3 levels were higher in the anterior lenticular pool than in the posterior. Levels of the Human Alpha-crystallin A chain and that of the Human Beta-crystallin B1 were higher in the posterior pool. The representative MS/ MS spectra of Beta-crystallin B1 and Human glyceraldehyde 3-phosphate dehydrogenase and identified PTMs with a-, b-, y-, B-Pi, and $\mathrm{y}$-Pi ion series that were identified either with MASCOT or ModiroTM are shown in Figure S2-S9.

\section{DISCUSSION}

Herein, UV absorbance differences at various anteroposterior sections of the human lens and that of the anterior and posterior lens capsules in the range of $240-400 \mathrm{~nm}$ were studied. It was observed that the posterior layers of the lens reveal higher absorption coefficients than the anterior layers at wavelengths shorter than $310 \mathrm{~nm}$ (data in Fig. 2). These results correspond to data reported in literature. In addition, it was observed that the posterior lens capsules show higher absorption in the UV-B and UV-C range than the anterior capsules (Fig. 3). Our data suggest that the epithelium of the anterior capsule does not exhibit significant UV absorbance (Fig. 4). It is noted, however, that we found only minor differences between the two age groups (40-49 years and 50-59 years) both at the level of individual parts of the lens or that of cumulative parameters.

To demonstrate the UV-filtering effect of the different layers of the lens, absorbance of the whole lens together with the lens capsules was calculated from the data presented above. First, the absorbance of the anterior capsule was computed, subsequently the absorbance of the anterior cortex was calculated and added to that of the anterior capsule. Analogously, absorbances of the nucleus, posterior cortex, and posterior capsule were added to that of the anterior layers, thus calculating the 

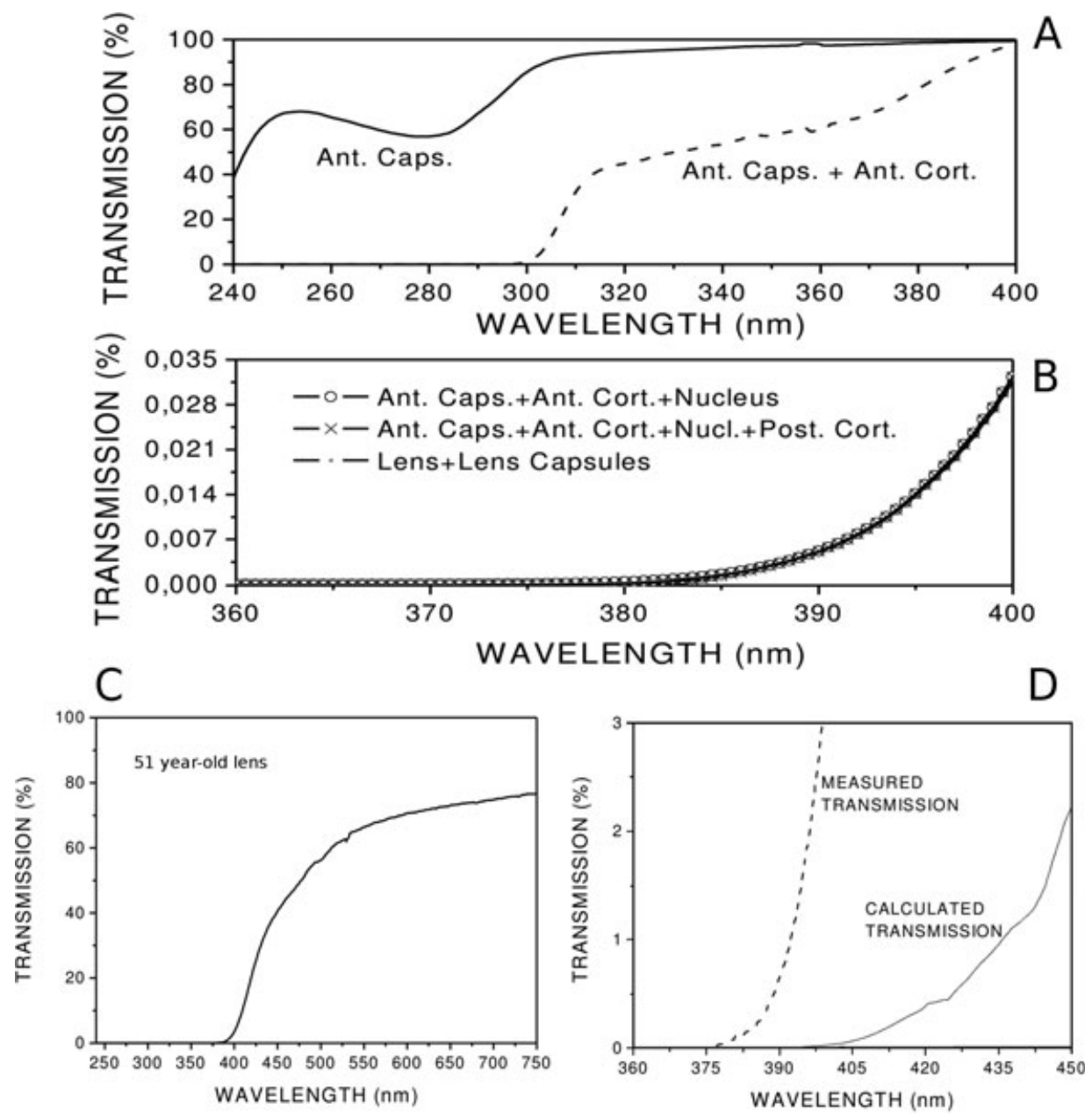

Figure 7. (A) Calculated transmission values of anterior capsule and that of the anterior capsule and the anterior cortex together (50-59 years age group). (B) Calculated transmission values of anterior capsule, anterior cortex and nucleus together, that of the anterior capsule, anterior cortex, nucleus and posterior cortex together and that of the whole lens with lens capsules. Note the considerable transmission loss of UV in the anterior parts of the lens. (C) Measured transmission of a 51-year-old whole human lens. (D) Comparison of the measured and calculated transmission values for the whole lens in the 360-450 nm wavelength range (data are obtained and shown along an enlarged scale from the intact whole lens shown in $\mathrm{C}$ and from calculated transmission values shown in B). Note that the actual values of the measured and calculated cut-off wavelengths are quite similar ( $376 \mathrm{vs} 390$ nm), although the curves show considerable displacement at greater transmission values, such as from $0.5 \%$.

absorbance of the whole lens. Figure 6 shows cumulative absorbance data as function of wavelength in the age group of $40-49$ years. It is evident that the nucleus mainly contributes to the whole absorbance by its thickness.

Similarly, transmission of the whole lens was calculated. Figure 7 shows that at wavelengths shorter than $290 \mathrm{~nm}$ the anterior capsule absorbs about $40 \%$ of the UV radiation and the transmission is reduced to approximately $0 \%$ by the rest of the lens structures. From the practical point of view, the transmission parameters of the investigated part of ocular system are also important. Accordingly, the transmission of the capsule, that of the whole lens and the complete lens capsule system have also been calculated (Fig. 7). Figure 7A shows that at wavelengths shorter than $290 \mathrm{~nm}$ the anterior capsule transmits about $60 \%$ of the UV radiation while the transmission is reduced to approximately $0 \%$ by the rest of the lens structures (Fig. 7B). To verify our measured and derived values, we determined the transmission of a whole lens using a special measuring arrangement. Figure $7 \mathrm{C}$ shows the transmission curve of a 51-year-old lens. The most important wavelength range was magnified (Fig. 7D) to compare the tendencies of the transmission curves concerning the whole lens obtained by direct measurement and indirect calculation. It can be seen clearly that the tendencies are similar, with a clearly observable displacement/difference of the curves which are due to the reflection and light scattering effects on surfaces of the investigated lens sections. While these effects are negligible in the case of a single lens layer $(60 \mu \mathrm{m})$ measurement, they are added up when the total lens transmission is calculated from the values of several lens sections. However, we were not able to remove these scattering effects in our transmission calculations for the whole lens.

To understand the rate of the absorption changes within the lens, the values of the absorption coefficients of the various layers at $280 \mathrm{~nm}$ are shown as function of anteroposterior depth in Figure. S1 (see supplementary data).

Studying protein expression differences between the anterior and posterior lenticular pools revealed that levels of human Betacrystallin B2 and A3 and that of glyceraldehyde 3-phosphate dehydrogenase were significantly higher in the anterior cortex of the lens whereas levels of Alpha-crystallin A chain and 
Beta-crystallin B1 were higher in the posterior segments. Crystallins are major lenticular proteins and serve a series of functions including chaperoning, contribution to transparency and refractive index, and determine lens structure and physicochemical properties. The alpha-crystallins are thought to play the roles of molecular chaperones and are members of the small heat shock protein family, moreover, they are supposedly associated with a number of neurological disorders. The beta/gamma-crystallin superfamily possesses well-defined $\mathrm{N}$ - and $\mathrm{C}$-terminal extensions, being responsible for their distinct biophysical and biochemical properties. Mutations in the beta/gamma-crystallin genes may lead to opacification of the lens.

The individual crystallins may play distinct roles under UV irradiation: Alpha-crystallin, a major constituent of the lens inhibits UV-light-induced aggregation of other lens proteins (27) and is thought to be a major target for UV irradiation due to its higher degree of unfolding (28). Earlier studies by Liang et al. $(18,19)$ have shown that tryptophan residues of alpha-crystallin are more exposed to UV radiation due to their greater degree of protein unfolding than those of beta or gamma-crystallins, and the unfolding of alpha-crystallin further increases with aging. Accordingly, as tryptophan residues are mainly responsible for the peak UV absorbance of the lens at $280 \mathrm{~nm}$, alpha-crystallin is suggested to play a major role in UV absorption compared with beta or gammacrystallins. Therefore, it is not surprising that increased amounts of alpha-crystallin in the posterior cortex may induce greater UV absorption than anterior parts enriched in beta-crystallin isoforms, which display lesser ability to absorb UV radiation. Moreover, sites of attachment of UV filters could be identified and linked to betaB1-crystallin (29). Gamma-crystallin D is an essential lens protein and exposure to UV-C is thought to perturb protein structure and probably leading to aggregation (30) and a gamma-crystallin fold may have been evolved to protect tryptophan from UV photodamage (31).

These crystallins may present the molecular basis for differences in the anterior and posterior UV absorbance because the higher degree of unfolding of Alpha-crystalline A and Beta-crystalline B1 chains may render the posterior part of the lens a more UV absorbing structure. The manifold increase of glyceraldehyde-3-phosphate dehydrogenase in the anterior section pool may be due to and reflect differences in carbohydrate metabolism within the lens. Interestingly, this enzyme was described as a target of UV-B (32).

It remains open whether differential crystallin localization underlies or reflects different optical or biological functions of the lens.

Acknowledgements-We acknowledge the contribution by the Verein zur Durchführung der wissenschaftlichen Forschung auf dem Gebiet der Neonatologie und Kinderintensivmedizin "Unser Kind". The authors are grateful for the support of the Hungarian Scientific Research Fund (OTKA, grant nos. T/F 043371 and K 67818). This work was partially supported by the Office for National Research and Technology (NKTH, project number CNK 78549).

Conflicts of interest-The authors have declared no conflict of interest.

\section{SUPPORTING INFORMATION}

Additional Supporting Information may be found in the online version of this article:
Table S1. Identification of lens protein spots by Mascot software including ion scores/mass errors and MS/MS peptides.

Table S2. Modifications of identified lens proteins (Modiro)

Table S3. Sequence coverage of all five individual proteins from different enzyme digestions, conditions and different search algorithms

Figure S1. The values of the absorption coefficients of the various layers at $280 \mathrm{~nm}$ are shown as function of anteroposterior depth.

Figure S2-S9. The representative MS/MS spectra of betacrystallin B1 and human glyceraldehyde 3-phosphate dehydrogenase and identified PTMs with a-, b-, y-, B-Pi, and y-Pi ion series are shown.

\section{REFERENCES}

1. Kolozsvári, L., A. Nógrádi, B. Hopp and Z. Bor (2002) UV absorbance of the human cornea in the 240- to 400-nm range. Invest. Ophthalmol. Vis. Sci. 43, 2165-2168.

2. Merriam, J. C. (1996) The concentration of light in the human lens. Trans. Am. Ophthalmol. Soc. 94, 803-918.

3. Zigman, S. (1993) Ocular light damage. Photochem. Photobiol. 57, 1060-1068.

4. Zigman, S., T. Paxhia, T. McDaniel, M. F. Lou and N. T. Yu (1991) Effect of chronic near-ultraviolet radiation on the gray squirrel lens in vivo. Invest. Ophthalmol. Vis. Sci. 32, 1723-1732.

5. Zigman, S. and T. Vaughan (1974) Near-ultraviolet light effects on the lenses and retinas of mice. Invest Ophthalmol. 13, 462-465.

6. Bochow, T. W., S. K. West, A. Azar, B. Munoz, A. Sommer and H R. Taylor (1989) Ultraviolet light exposure and risk of posterior subcapsular cataracts. Arch. Ophthalmol. 107, 369-372.

7. Cruickshanks, K. J., B. E. Klein and R. Klein (1992) Ultraviolet light exposure and lens opacities: the Beaver Dam Eye Study. Am. J. Public Health 82, 1658-1662.

8. Dolin, P. J. (1994) Ultraviolet radiation and cataract: a review of the epidemiological evidence. Br. J. Ophthalmol. 78, 478-482.

9. Zigman, S., M. Datiles and E. Torczynski (1979) Sunlight and human cataracts. Invest. Ophthalmol. Vis. Sci. 18, 462-467.

10. Boettner, E. A. and J. R. Wolter (1962) Transmission of the Ocular Media. Invest. Ophthalmol. Vis. Sci. 1, 776-783.

11. Gaillard, E. R., L. Zheng, J. C. Merriam and J. Dillon (2000) Agerelated changes in the absorption characteristics of the primate lens. Invest. Ophthalmol. Vis. Sci. 41, 1454-1459.

12. Dillon, J., L. Zheng, J. C. Merriam and E. R. Gaillard (1999) The optical properties of the anterior segment of the eye: implications for cortical cataract. Exp. Eye Res. 68, 785-795.

13. Truscott, R. J., A. M. Wood, J. A. Carver, M. M. Sheil, G. M. Stutchbury, J. Zhu and G. W. Kilby (1994) A new UV-filter compound in human lenses. FEBS Lett. 348, 173-176.

14. Hood, B. D., B. Garner and R. J. Truscott (1999) Human lens coloration and aging. Evidence for crystallin modification by the major ultraviolet filter, 3-hydroxy-kynurenine O-beta-D-glucoside. J. Biol. Chem. 274, 32547-32550.

15. Bova, L. M., M. H. Sweeney, J. F. Jamie and R. J. Truscott (2001) Major changes in human ocular UV protection with age. Invest. Ophthalmol. Vis. Sci. 42, 200-205.

16. Kyselova, Z. (2011) Mass spectrometry-based proteomics approaches applied in cataract research. Mass Spectrom. Rev. 30, 1173-1184.

17. Yao, Z., H. Yu, D. Xuan, Q. Sha, J. Hu and J. Zhang (2010) Analysis of protein-protein interactions and proteomic profiles of normal human lenses. Curr. Eye Res. 35, 605-619.

18. Liang, J. N., U. P. Andley and L. T. Chylack, Jr. (1985) Spectroscopic studies on human lens crystallins. Biochim. Biophys. Acta 832, 197-203.

19. Liang, J. N., S. K. Bose and B. Chakrabarti (1985) Age-related changes in protein conformation in bovine lens crystallins. Exp. Eye Res. 40, 461-469. 
20. Diao, W. F., H. Hoger, W. Q. Chen, A. Pollak and G. Lubec (2007) Estrous-cycle-dependent hippocampal levels of signaling proteins. Hippocampus 17, 563-576.

21. Langen, H., P. Berndt, D. Roder, N. Cairns, G. Lubec and M. Fountoulakis (1999) Two-dimensional map of human brain proteins. Electrophoresis 20, 907-916.

22. Winding, C., Y. Sun, H. Höger, H. Bubna-Littitz, A. Pollak, P. Schmidt and G. Lubec (2011) Serine/threonine-protein phosphatase $1 \propto$ levels are paralleling olfactory memory formation in the CD1 mouse. Electrophoresis 32(13), 1675-1683. DOI: 10.1002/elps. 201000615

23. Chen, W. Q., S. U. Kang and G. Lubec (2006) Protein profiling by the combination of two independent mass spectrometry techniques. Nat. Protoc. 1, 1446-1452.

24. Kang, S. U., S. Heo and G. Lubec (2011) Mass spectrometric analysis of GABA-A receptor subtypes and phosphorylations from mouse hippocampus. Proteomics 11, 2171-2181. DOI: 10.1002/pmic.201000374.

25. Kang, S. U. and G. Lubec (2011) Determination of in-gel protein concentration by a ninhydrin-based method. Proteomics 11, 481-484.

26. Krag, S. and T. T. Andreassen (2003) Mechanical properties of the human posterior lens capsule. Invest. Ophthalmol. Vis. Sci. 44, 691-696.
27. Lee, J. S., J. H. Liao, S. H. Wu and S. H. Chiou (1997) Alpha-Crystallin acting as a molecular chaperonin against photodamage by UV irradiation. J. Protein Chem. 16, 283-289.

28. Weinreb, O., M. A. van Boekel, A. Dovrat and H. Bloemendal (2000) Effect of UV-A light on the chaperone-like properties of young and old lens alpha-crystallin. Invest. Ophthalmol. Vis. Sci. 41, 191-198.

29. Aquilina, J. A. and R. J. Truscott (2002) Identifying sites of attachment of UV filters to proteins in older human lenses. Biochim. Biophys. Acta 1596, 6-15.

30. Wang, S. S. and W. S. Wen (2010) Examining the influence of ultraviolet $\mathrm{C}$ irradiation on recombinant human gammaD-crystallin. $\mathrm{Mol}$ Vis. 16, 2777-2790.

31. Chen, J., P. R. Callis and J. King (2009) Mechanism of the very efficient quenching of tryptophan fluorescence in human gamma D- and gamma S-crystallins: the gamma-crystallin fold may have evolved to protect tryptophan residues from ultraviolet photodamage. Biochemistry 48, 3708-3716.

32. Schmidt, J., C. Schmitt, M. Kojima and O. Hockwin (1992) Biochemical and morphological changes in rat lenses after long-term UV B irradiation. Ophthalmic Res. 24, 317-325. 


$$
\text { II. }
$$




\title{
Age-Related Absorption of the Human Lens in the Near-Ultraviolet Range
}

\author{
Viktor Pajer $^{1 \dagger}\left(D\right.$, Ferenc Rárosi ${ }^{2}$, Lajos Kolozsvári ${ }^{3}$, Béla Hopp ${ }^{1}$ and Antal Nógrádi ${ }^{4}$ \\ ${ }^{1}$ Department of Optics and Quantum Electronics, Faculty of Science and Informatics, University of Szeged, Szeged, \\ Hungary \\ ${ }^{2}$ Department of Medical Physics and Informatics, Faculty of Medicine, University of Szeged, Szeged, Hungary \\ ${ }^{3}$ Department of Ophthalmology, Faculty of Medicine, Albert Szent-Györgyi Clinical Centre, University of Szeged, Szeged, \\ Hungary \\ ${ }^{4}$ Department of Anatomy, Faculty of Medicine, University of Szeged, Szeged, Hungary \\ Received 22 May 2019, accepted 19 November 2019, DOI: 10.1111/php.13199
}

\begin{abstract}
The purpose of the present study was to determine the age dependence of the ultraviolet (UV) absorption of the different parts of the human crystalline lens. Cryostat sections of human cadaveric lenses $(60 \mu \mathrm{m})$ were cut. The $U V$ absorbance of nine samples, derived from different parts of the lens, was determined using a Shimadzu scanning spectrophotometer. The absorbance of the anterior and posterior lens capsules was measured separately. The absorption coefficients were calculated from the measured absorbance and values taken at 280 as well as at $360 \mathrm{~nm}$ were compared statistically. ANCOVA analysis of the values taken at 280 and at $360 \mathrm{~nm}$ wavelengths shows that correlation between the absorption coefficients and age can be found only in the case of the posterior layers. These results suggest a differential age-dependent increase of the $\mathrm{UV}$ absorption of the posterior layers compared to the anterior ones and can be related to the differential protein expression in the anterior and posterior parts. Posterior crystalline lens capsules have higher absorption coefficients than the anterior ones regardless of age.
\end{abstract}

\section{INTRODUCTION}

The human lens has an effective capacity to absorb UV radiation (1-3) and along with the other parts of the eye, plays an important role in preventing the retina from ocular light damage. Several studies have provided evidence that higher UV exposure, as well as the age, increase the prevalence of certain eye diseases (4-7), especially that of different types of cataract (8-13). The age dependence of light absorption by the human crystalline lens has been the center of interest for a long time. Weale (1) found a systematic age-dependent increase in the absorbance both in the visible light and near-UV range. In accordance with this, transmission spectra of human lenses $(3,14)$ of different ages show that the older lenses transmit less amount of light of wavelengths longer than $300 \mathrm{~nm}$. Since the lens grows continuously, higher absorption can be caused by the longer light path, but

*Corresponding author email: nogradi.antal@med.u-szeged.hu (Antal Nógrádi) †Current address: ELI-ALPS, Szeged, Hungary

(C) 2019 The Authors. Photochemistry and Photobiology published by Wiley Periodicals, Inc. on behalf of American Society for Photobiology

This is an open access article under the terms of the Creative Commons Attribution License, which permits use, distribution and reproduction in any medium, provided the original work is properly cited. physiological yellowing of the older lens can also contribute to the increased absorbance (15).

It is known that the various parts of the lens absorb UV light differentially, that is, absorbance increases in anteroposterior direction $(2,16,17)$. On the other hand, studies on age-related changes do not provide an explanation how this absorption gradient varies with age. Moreover, very little is known about the absorption capacity of the lens capsules and their age-related changes (18).

Therefore, the aim of the present study was to determine the age dependence of the UV absorption of the different parts of the human crystalline lens.

\section{MATERIALS AND METHODS}

Cryostat sectioning. A total of 38 human lenses, from the age of 7 to 81 years, were used for measuring the age-related changes of the UV absorbance of the human lens. The human lenses were used with the approval of the Human Ethics Committee of University of Szeged, Albert Szent-Györgyi Clinical Centre and the study was conducted in accordance with the provisions of Declaration of Helsinki for experimentation involving human tissue. Lenses were obtained with a postmortem delay of $6 \mathrm{~h}$ and were stored at $-70^{\circ} \mathrm{C}$ until used.

Only those lenses which showed no cataract formation upon postmortem examinations were used for measurements. To determine the absorption of the various parts of the lens, the same procedure was used as reported in our earlier paper (17). Briefly, $60-\mu \mathrm{m}$ thick consecutive sections were cut in a cryostat (Leica $1850 \mathrm{CM}$ ) at $-18^{\circ} \mathrm{C}$. Before sectioning, the anterior and posterior lens capsules were removed and stored in physiological saline separately. During this process, some of the capsules were damaged and could not be used for measurements, thus altogether 19 anterior and 27 posterior capsules were examined. During cryostat sectioning a total of nine sections were kept for measurements in an anteroposterior order: two samples from the anterior cortex (sample 1 and 2), five samples from the nucleus (samples 3-7) and two samples from the posterior cortex (samples 8 and 9), for further details see Fig. 1 and our earlier work (17).

As a result of the sectioning method, samples 4-7 always derived from the juvenile nucleus (19-21), thus the age-related changes of the sections taken from the nucleus are comparable, despite the fact that the thickness of the full lens increases with age.

Following UV measurements, cryostat cross sections were cut from the anterior and posterior capsules to determine the thickness of the capsules. Thickness of the anterior capsules varied from 21 to $26 \mu \mathrm{m}$, whereas that of the posterior ones were relatively constant in the range of 8 and $11 \mu \mathrm{m}$.

Spectrophotometric measurements. The dependence of the UV absorbance of the samples as function of wavelength was measured by a scanning spectrophotometer (model UV-2101PC UV- VIS; Shimadzu, 

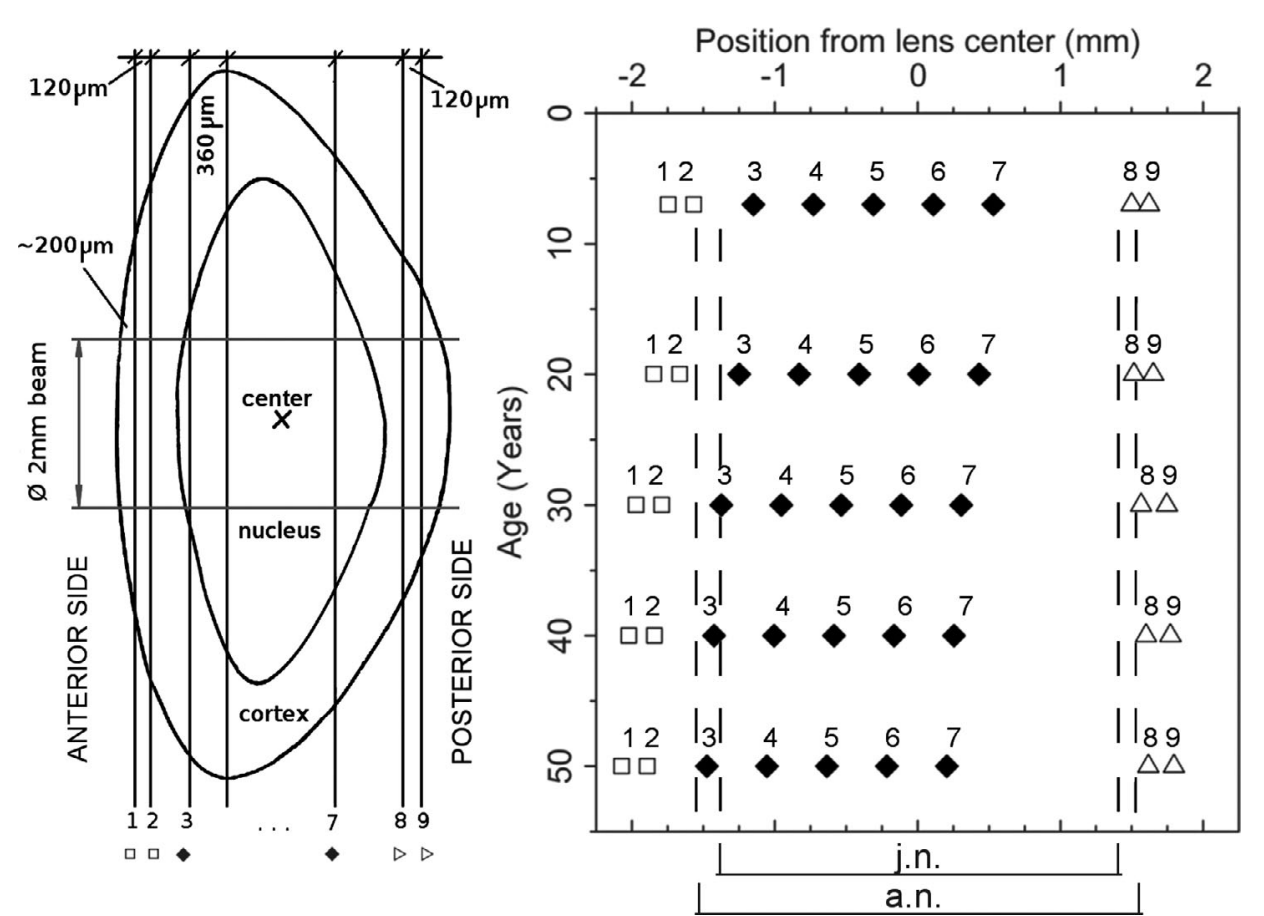

Figure 1. Schematic view of sections' position inside the lens (left) and the calculated position from the lens center (right). Different regions of an aged nucleus are indicated (j.n.: juvenile nucleus, a.n.: adult nucleus) based on literature data.

Kyoto, Japan) at room temperature. The absorbances and the absorption coefficients of the samples were determined as reported in our earlier work (17). The round $2 \mathrm{~mm}$ beam used for spectrophotometric measurements made it possible to measure only the central regions of the samples (Fig. 1). The investigated wavelength range was $240-400 \mathrm{~nm}$, as in the range below $240 \mathrm{~nm}$, the absorbance values often approached or reached the upper limit of the device.

Statistical analysis. Descriptive statistics were calculated for each layer separately. Continuous variables were expressed as mean $\pm \mathrm{SD}$. The tendency between age and absorption coefficients at $280 \mathrm{~nm}$ as well as at $360 \mathrm{~nm}$ was characterized with Pearson correlation coefficients and significance tests for correlation for each layer separately. Analysis of covariance (ANCOVA) model with factor layer and covariate age was used for further statistical analysis. A $P$-value $<0.05$ was considered to be statistically significant. The calculations were computed through the use of IBM SPSS version 24.

\section{RESULTS}

\section{Absorption spectrum of lens sections}

The UV absorption of the lenses was examined in seven different age groups: 7-19 $(n=3), 20-29(n=3), 30-39(n=6), 40$ $49(n=10), 50-59(n=9), 60-69(n=4)$ and $70-81(n=3)$ years. There was only one lens younger than 10 years old available so the age range of the first group was extended to include this sample. Due to the lack of samples, a similar expansion of the 70-81 years age group was performed. For statistical analysis, the age was considered as a continuous value and no age groups were formed. Figure 2 shows the averaged absorption coefficients of the nine sample layers as function of wavelength in three different age groups. The absorption spectrum slightly increases toward the shorter wavelengths and has a maximum at the wavelength of $280 \mathrm{~nm}$. It can be seen that value of the maximum absorption increases in the anteroposterior direction, except in the case of the youngest group (7-19 years), in which the increasing tendency is not explicit and the samples 4-7 have almost equal absorption capacity.

The reported absorbance spectra of the human lens show another absorption peak around $360 \mathrm{~nm}(1,2,22)$. Figure 3 presents the averaged absorption coefficients of three age groups in the $300-400 \mathrm{~nm}$ wavelength range. For clarity, data related to Samples 1, 3, 5, 7 and 9 only are shown. The posterior layers have higher absorption capacity, but the increasing tendency is not as explicit as in the case of the peak at $280 \mathrm{~nm}$. It is also noticeable that only the spectra of the youngest lenses show a small peak around $360 \mathrm{~nm}$ meanwhile the absorption is slightly decreased at $320 \mathrm{~nm}$. As the detection limit of the spectrophotometer was calibrated in a way to measure the peak at $280 \mathrm{~nm}$, the absorbance in the UV-A range presumably could have been detected with lower precision. Therefore, the fine absorption changes are visualized to a lesser extent compared with the previously published results.

\section{Age-dependent changes of absorption}

Although UV radiation at wavelengths shorter than $300 \mathrm{~nm}$ is presumably absorbed by the cornea, the $280-\mathrm{nm}$ wavelength, where the absorption spectrum has a maximum, offers a plausible way to visualize accurately the age-related changes of the UV absorption of the lens. On the other hand, as the cornea is not able to fully absorb the UV radiation longer than $320 \mathrm{~nm}$, it is physiologically also relevant to investigate the age-related changes in the UV-A range. The averaged absorption coefficients at $280 \mathrm{~nm}$ as well as at $360 \mathrm{~nm}$ of the anterior and posterior cortex and that of the nucleus were calculated in the following way: average of the values of samples 1 and 2 provided the absorption coefficient of the anterior cortex that of the nucleus was calculated from the data of layers $3-7$, and the average of the spectra 

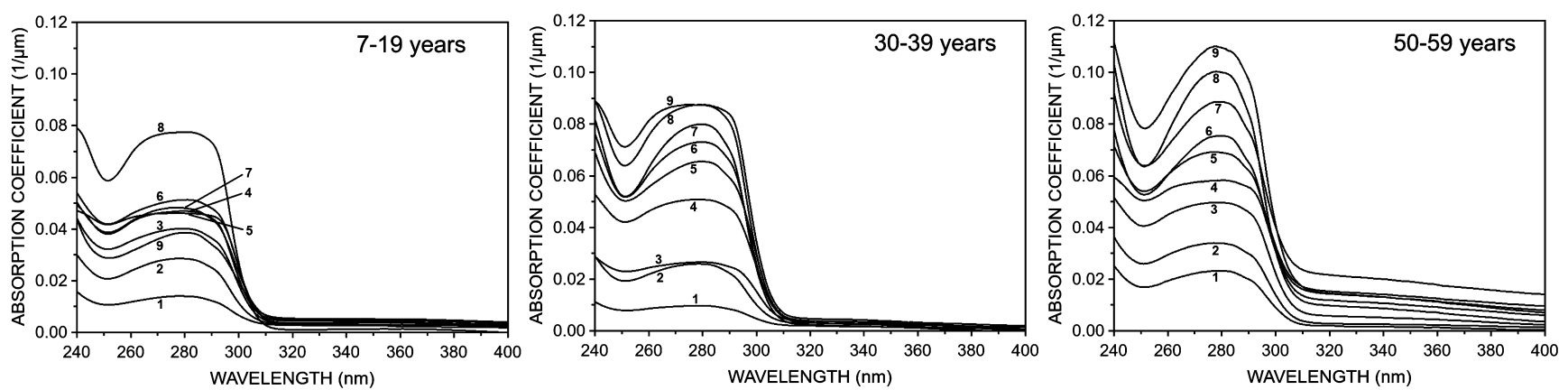

Figure 2. Averaged absorption coefficients of the nine sample layers as function of wavelength in three different age groups.
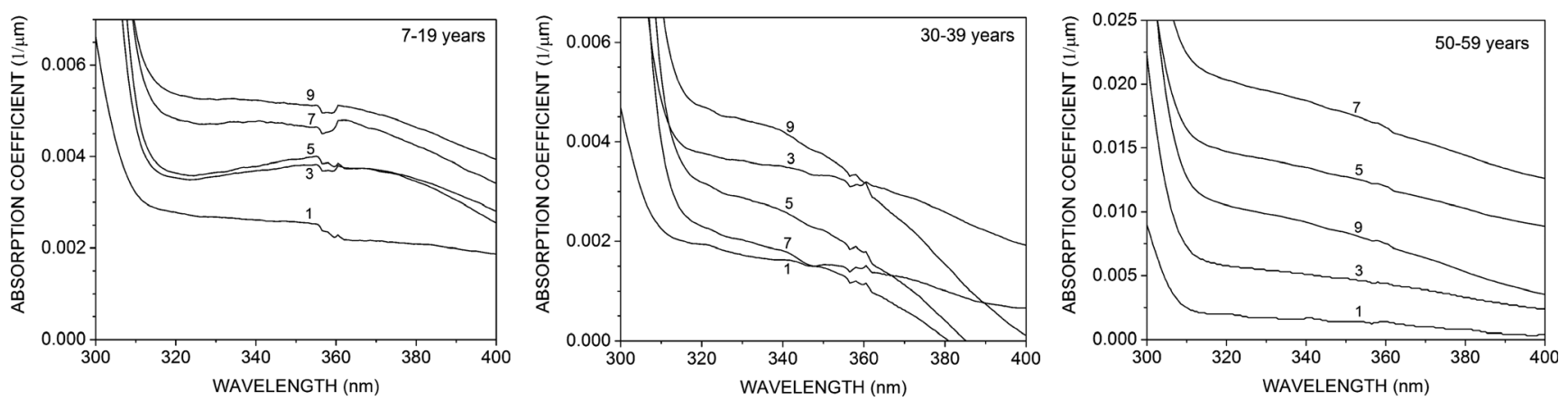

Figure 3. Averaged absorption coefficients of five sample layers as function of wavelength in three different age groups, in the 300 to $400 \mathrm{~nm}$ range.

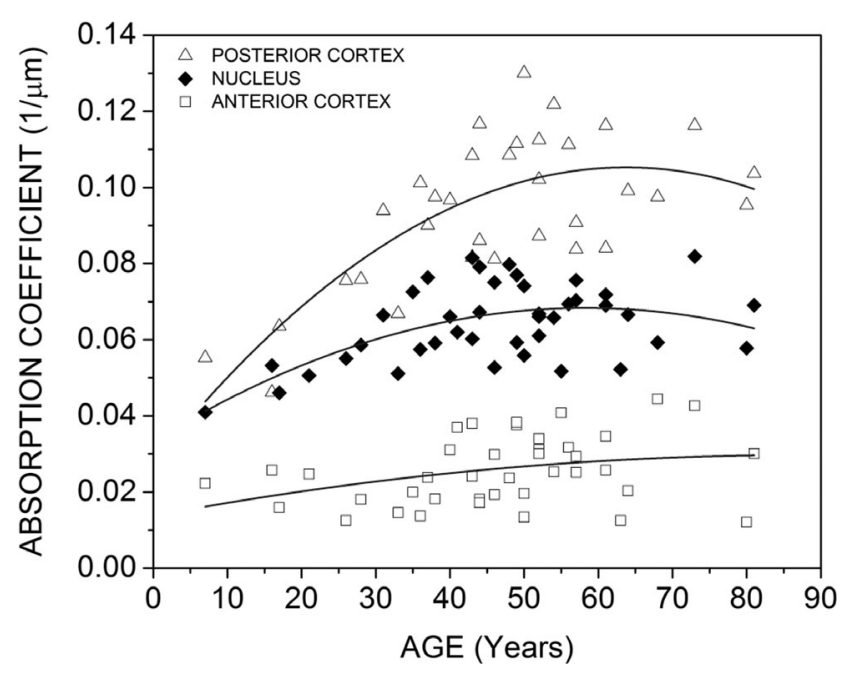

Figure 4. Averaged values of absorption coefficients taken at $280 \mathrm{~nm}$ as function of age. Solid lines show the second order polynomial fit. Agerelated changes of the posterior cortex are the most noticeable.

of samples 8 and 9 resulted in the absorption spectra of the posterior cortex. These calculations were performed on every single lenses. The averaged values of the absorption coefficient at $280 \mathrm{~nm}$ as function of age are shown in Fig. 4. The second order polynomial fit clearly shows that the age-related changes of the posterior cortex are the most pronounced compared to the other two parts. The absorption increases until the age of 60 where it reaches a plateau and slightly decreases after the age of 70 .

Averaged absorption coefficients taken at $360 \mathrm{~nm}$ are presented in Fig. 5. Both the nucleus and posterior cortex show increasing absorption capacity with age but changes of the nucleus are the most noticeable.

To determine exactly how the age affects the absorption properties of the different layers and whether this effect is significant, statistical analysis was performed. Values of the absorption coefficients taken at $280 \mathrm{~nm}$ of the nine sample layers of every single lens were collected. The ANCOVA model revealed a borderline significant interaction between age and layer $(P=0.057)$. This result is significant at $10 \%$ level, with respect to the moderate sample size, this suggests that the effect of age depends on the layer. Changes of coefficient values as function of age were examined for each layer. Table 1 shows the correlation coefficients between age and the absorption coefficients at $280 \mathrm{~nm}$ and the results of significance tests for each layer. It can be seen that the correlation was significant in layers 6 $(R=0.371, \quad P=0.022), \quad 7 \quad(R=0.49, \quad P=0.002) \quad$ and $\quad 9$ $(R=0.363, P=0.041)$.

Figure 6 shows the values of the absorption coefficients taken at $280 \mathrm{~nm}$ of samples 6,7 and 9 as function of age. Indeed, these results demonstrate a weak, but significantly increasing tendency of absorption.

In the case of absorption coefficients taken at $360 \mathrm{~nm}$, the ANCOVA statistical model revealed a significant interaction between age and layer $(P<0.01)$, showing that the effect of age depends on the layer position in the UV-A range. Table 2 displays the correlation coefficient values between age and the absorption coefficient. The correlation proved to be significant in layers $5(R=0.379, P=0.047), 6(R=0.614, P=0.000)$ and 7 $(R=0.676, P=0.000)$. In Fig. 7, the absorption coefficients taken at $360 \mathrm{~nm}$ of samples 5, 6 and 7 as function of age are presented. Indeed, the values show the increased absorption capacity of the nuclear region of the aged lenses. 


\section{Viktor Pajer et al.}
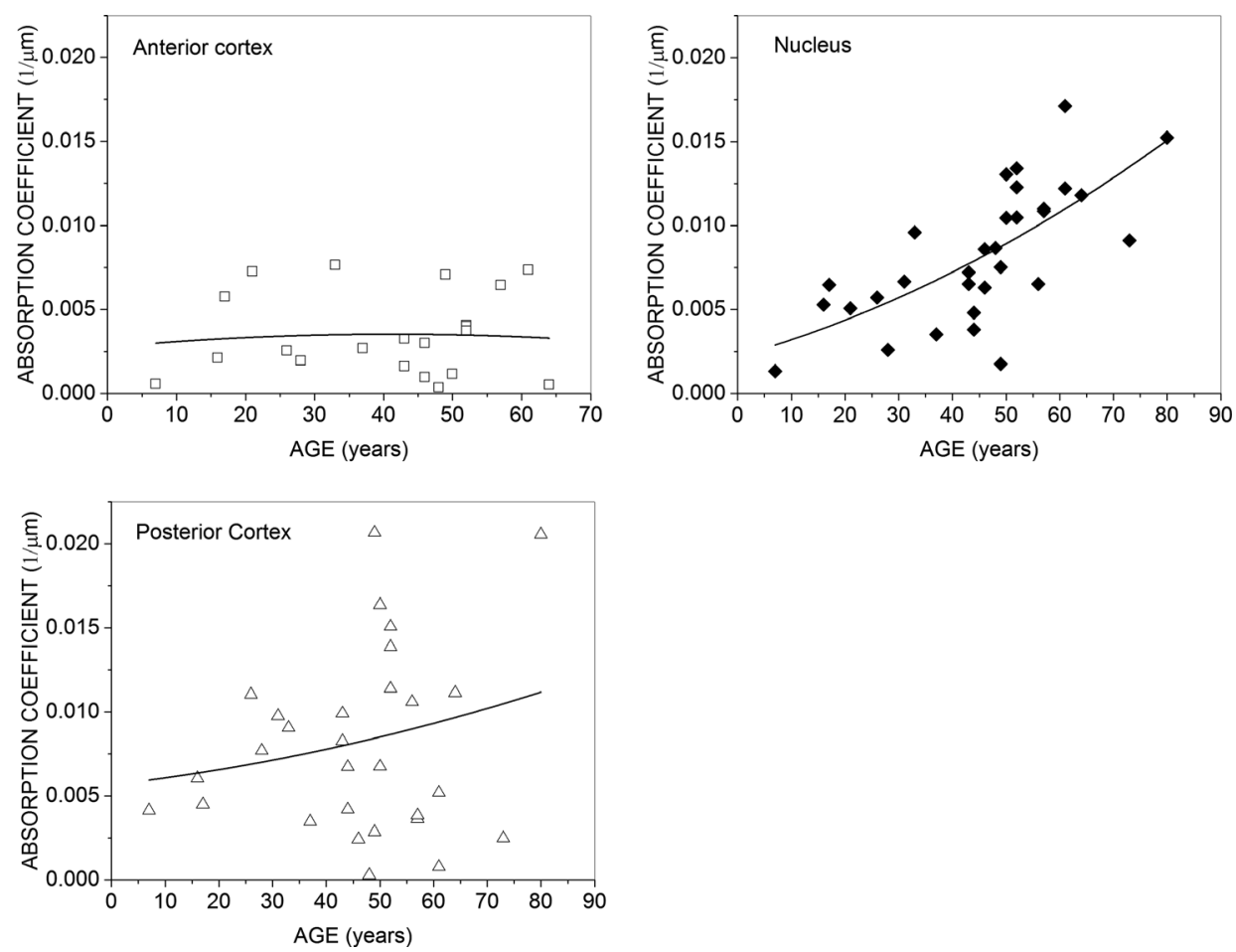

Figure 5. Averaged values of absorption coefficients taken at $360 \mathrm{~nm}$ as function of age. Solid lines show the second order polynomial fit. Age-related changes of the nucleus are the most pronounced.

Table 1. Test $P$-values of the ANOVA analysis related to the absorption coefficients taken at $280 \mathrm{~nm}$.

\begin{tabular}{lccccccccc}
\hline Sample layers & 1 & 2 & 3 & 4 & 5 & 6 & 7 & 8 & 9 \\
\hline$R$ & 0.056 & 0.16 & 0.158 & 0.136 & 0.215 & 0.371 & 0.49 & 0.096 & 0.363 \\
$P$-values & 0.737 & 0.337 & 0.343 & 0.414 & 0.207 & $\mathbf{0 . 0 2 2}$ & $\mathbf{0 . 0 0 2}$ & 0.582 & $\mathbf{0 . 0 4 1}$
\end{tabular}

Values $<0.05$ were considered to be statistically significant (bold-faced values).
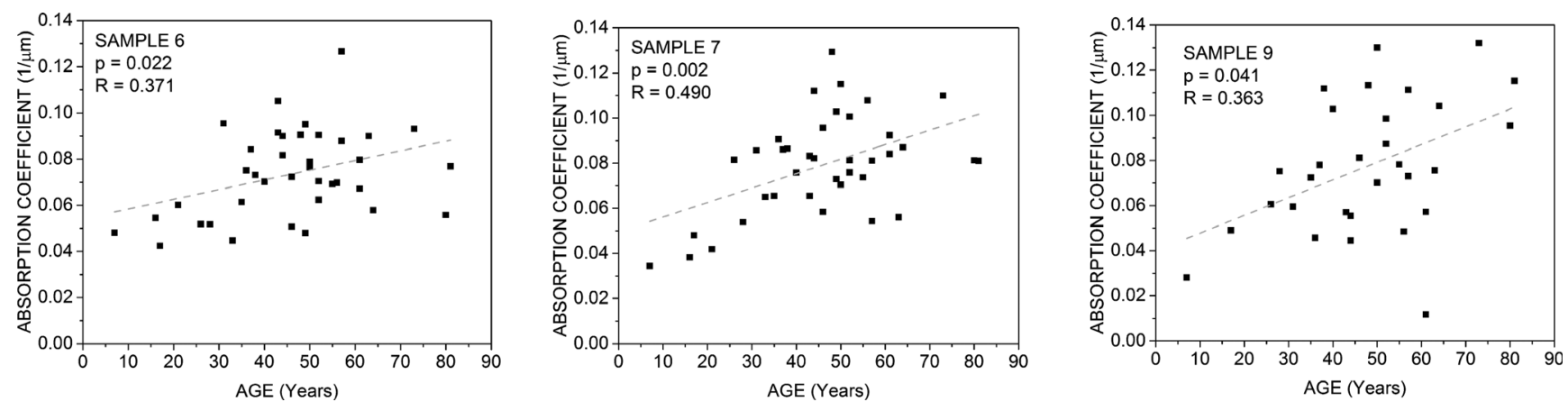

Figure 6. Absorption coefficients at $280 \mathrm{~nm}$ of the three age-dependent sample layers are shown as function of age. Significant $R$ and $P$-values are indicated.

Table 2. Test $P$-values of the ANOVA analysis related to the absorption coefficients taken at $360 \mathrm{~nm}$.

\begin{tabular}{lccccccccc}
\hline Sample layers & 1 & 2 & 3 & 4 & 5 & 6 & 7 & 8 & 9 \\
\hline$R$ & -0.16 & -0.32 & 0.129 & 0.337 & 0.379 & 0.614 & 0.676 & 0.195 & 0.261 \\
$P$-values & 0.480 & 0.165 & 0.547 & 0.079 & $\mathbf{0 . 0 4 7}$ & $\mathbf{0 . 0 0 0}$ & $\mathbf{0 . 0 0 0}$ & 0.311 & 0.266
\end{tabular}

Values $<0.05$ were considered to be statistically significant (bold-faced values). 

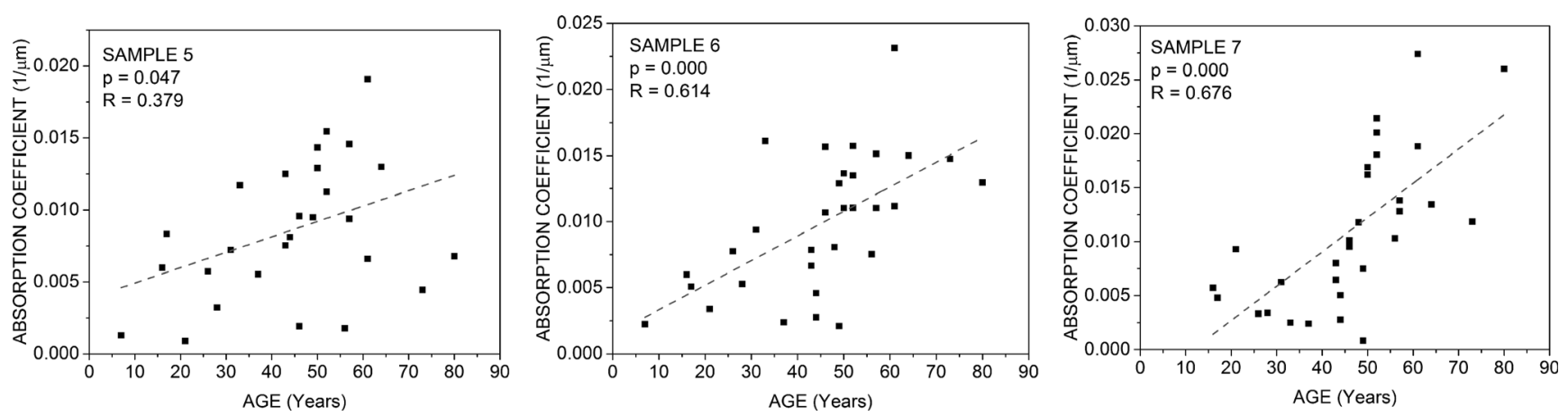

Figure 7. Absorption coefficients at $360 \mathrm{~nm}$ of the three age-dependent sample layers are shown as function of age. Significant $R$ and $P$-values are indicated.

\begin{abstract}
Absorption of the lens capsules
Owing to the small number of the samples, the averaged absorption coefficients of the lens capsules were calculated only in five different age groups: 7-19 years, 20-39 years, 40-49 years, 5059 years and above the age of 60 . Again, for statistical analysis, the age was considered as a continuous value and no age groups were formed. Regardless of age, we found that the posterior capsules show higher absorption than the anterior ones (Fig. 8) but further statistical analysis (ANCOVA) showed that this difference is not significant $(P=0.198)$. The age showed no significant effect on absorption coefficients at $280 \mathrm{~nm}(P=0.18)$.
\end{abstract}

\section{DISCUSSION}

We examined the age-related UV absorption changes of the various parts of the human lens in an expanded age range (781 years), and it was found that there is an age-independent absorption gradient in the anteroposterior direction inside the lens. Only the youngest lenses showed slightly different tendency in the UV-C range which can be explained by the remodeling of the lens in this age (20) when the aspect ratio significantly changes and the primary fiber cell shortening happens. The results of the statistical analysis showed a significant correlation between the absorption coefficient taken at $280 \mathrm{~nm}$ and the age only in the case of the posterior layers $6(R=0.371, P=0.022)$, $7(R=0.49, P=0.002)$ and $9(R=0.363, P=0.041)$. At $360 \mathrm{~nm}$, significant correlation was found between age and absorption of nuclear layers $5(R=0.379, P=0.047), 6$ $(R=0.614, P=0.000)$ and $7(R=0.676, P=0.000)$. The anterior capsules have lower absorption coefficients than the posterior ones in each age groups; however, this difference is not significant and no age-related changes were found in these cases. This indicates that the age has no effect on the absorption properties of the lens capsules.

Most studies treat the cortex as a whole, since the anterior and posterior sides consist of the same elongated fibers. However, several results suggest that there is a difference in protein expression levels along the visual axis. Spectroscopically obtained Carr-Purcell-Meiboom-Gill data suggest spatial and age-dependent variations in crystallin content (23). Water content in the posterior pole is lower than in the anterior pole (24), indicating higher protein concentration in the posterior part. Levels of alpha-crystallin A and beta-crystallin B1 chains are higher in the posterior layers of the lens (17). The concentration of alphacrystallin also increases with age at the expense of beta- and gamma-crystallins (25). These findings suggest that the age-related changes of the absorption at $280 \mathrm{~nm}$ are strongly related to the age-dependent protein expression levels. The water soluble protein fraction decreases after middle age, resulting in decreased
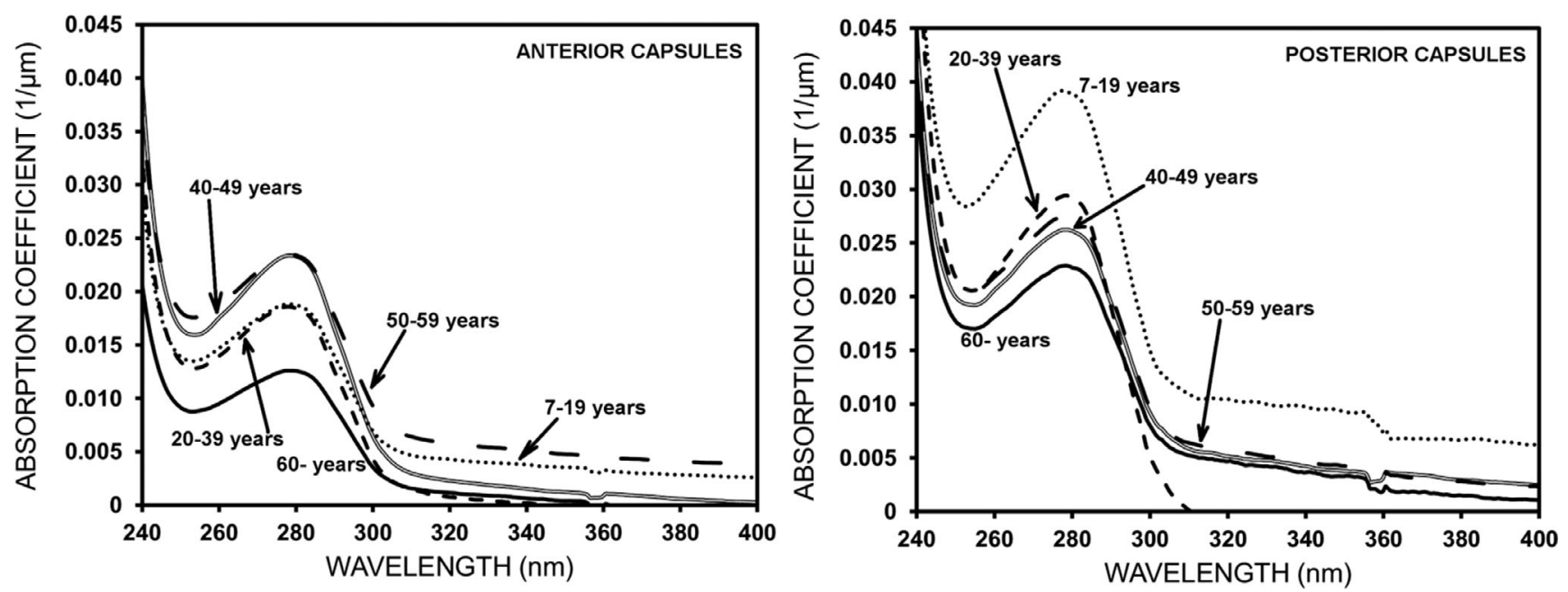

Figure 8. Averaged absorption coefficients of the capsules as function of wavelength. The curves are related to the indicated age groups. 
absorption at $280 \mathrm{~nm}$ wavelength (26) which can explain the plateau around the age of 60 in the absorption coefficient of the posterior cortex and of the nucleus (Fig. 4). The UV-filter compounds, such as 3-hydroxykynurenine glucoside (3OHKG) or 3hydroxykynurenine (3OHKyn) are responsible for the absorption in the UV-A and -B ranges. The concentration of all the tryptophan metabolites shows a decreasing tendency with age (27), except the glutathione adduct of 3OHKG. On the other hand, the levels of protein bound UV-filters especially that of $3 \mathrm{OHKG}$ in the nucleus, are significantly increased after middle age (28). These age-dependent changes of $3 \mathrm{OHKG}$ are in agreement with the enhanced absorption of the lenses older than 50 years (Figs. 5 and 6) and can explain the significant correlation.

The age-related results found in the literature are taken from the entire lens or lens homogenates, except the study of Gaillard et al. (2). To determine the UV transmission of the whole lens, the averaged cumulative absorbance was calculated in each age groups. The absorption values of the discarded segments were considered to be equal to the absorbance values of the samples taken in front of the unused sections. The measured absorbance resulted from the absorption and scattering processes together. Although the samples were placed between quartz plates in a drop of isotonic saline, the amount of light scattering through the lens sections is presumably still higher than in the case of the measurements related to the whole lens. When the cumulative absorbance is calculated, the effect of a relatively small difference in the scattering process can be highly enhanced due to the large number of lens sections ( $n=60-70$ with $60 \mu \mathrm{m}$ thickness). In order to take into account this process, $2 \%$ of the incoming radiation was considered as scattered light in each step and the absorbance was reduced according to this. Then the transmission was calculated using the following formula:

$$
T=100 * 10^{(-A b s c)}
$$

where $T$ is the percent transmission and Absc is the corrected cumulative absorbance.

Figure 9 shows the transmission of the whole lens without the capsules in the case of seven different age groups. It can be seen that the youngest lenses transmits about $2 \%$ of the incoming light above $310 \mathrm{~nm}$, while the older lenses allow very little transmission (approximately 0.1\%) in the near-UV range. These absorption changes in the UV-B range is presumably caused by the age-dependent concentration of UV-filter molecules $(27,28)$ and are in agreement with the data found in the literature $(3,29,30)$. It can be also noticed that transmission of the lenses in the 7-19 years old age group shows a fine peak at wavelength of $320 \mathrm{~nm}$. This corresponds to the local minimum in the absorbance spectrum of young lenses (1 and 2). The transmission of lenses with different age shows similar features (30). Both Gaillard et al. (2) and Cooper and Robson (22) found that the peak at $360 \mathrm{~nm}$ shifts toward the shorter wavelengths with age and the minimum vanishes which can explain the above phenomena.
The same changes can be observed in the absorption spectrum of lens sections derived from individual lenses (Figure S1) and the averaged curves also show similar tendency (Fig. 3). To prove whether any age-dependent changes occur at this wavelength, statistical analysis was performed and it was found that almost all the nuclear layers show significant correlation with age (Table 3). These findings suggest that the absorption of the aged nucleus is increased at this wavelength; therefore, the transmission window can be observed in the case of the 7-19 years old group only. Interestingly, other studies $(3,14)$ found that the transmission of the lens is always increased around $320 \mathrm{~nm}$, independently of age. This discrepancy could be due to either the different measurement techniques (absorbance of lens sections compared to the transmission of the whole lens) or the detection limit of the spectrophotometer which was calibrated to the $280 \mathrm{~nm}$ peak value.

The averaged transmission curves are in good agreement with the data found in literature $(3,30)$. On the other hand, the measured transmission of individual lenses shows high variability. These differences shall be reflected by the absorbance of the lens sections too. Both in the 7-19 and 50-59 years age groups, the layers derived from individual lenses with the same age have significantly different absorption capacities (see Figure S2). Standard deviation (SD) values of absorption coefficients taken at $280 \mathrm{~nm}$ as well as at $360 \mathrm{~nm}$ also confirm that the variability is relatively high within the groups, especially that of the anterior layers at the longer wavelength. The SD values together with the means are presented in Tables S1 and S2.

Averaged transmission of lens capsules was calculated as well but no compensation was made for light scattering. In Fig. 10, it can be seen that below $300 \mathrm{~nm}$, the anterior and posterior capsules absorb approximately $30-40 \%$ and $20 \%$ of the incident UV

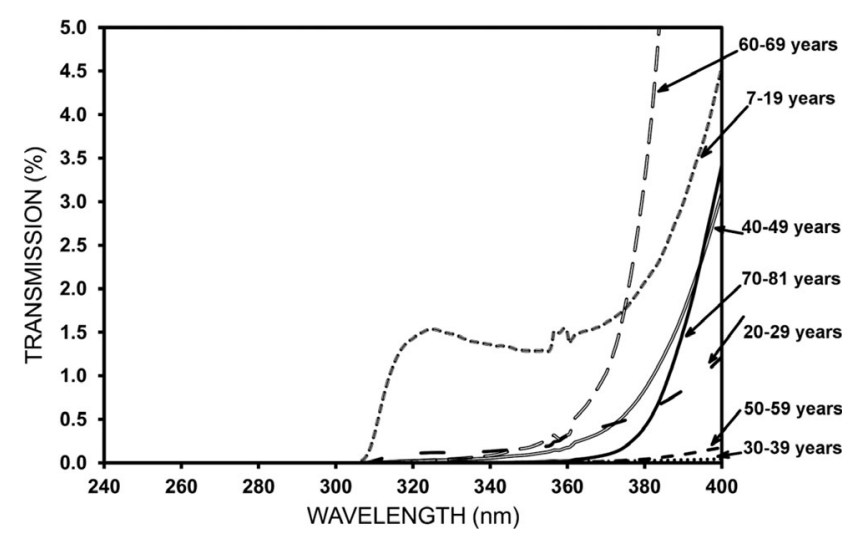

Figure 9. Calculated transmission of the full lens as function of the wavelength. Note the increased transmission of very young lenses at wavelength above $310 \mathrm{~nm}$. The older lenses possess very low capacity to transmit near UV light.

Table 3. Test $P$-values of the ANOVA analysis related to the absorption coefficients taken at $320 \mathrm{~nm}$.

\begin{tabular}{lccccccccc}
\hline Sample & 1 & 2 & 3 & 4 & 5 & 6 & 7 & 9 \\
\hline$R$ & 0.092 & -0.042 & 0.033 & 0.422 & 0.044 & 0.720 & 0.706 & 0.247 \\
$P$-value & 0.692 & 0.853 & 0.091 & $\mathbf{0 . 0 2 0}$ & $\mathbf{0 . 0 1 8}$ & $\mathbf{0 . 0 0 0}$ & $\mathbf{0 . 0 0 0}$ & 0.197 \\
\hline
\end{tabular}

Values $<0.05$ were considered to be statistically significant (bold-faced values). 

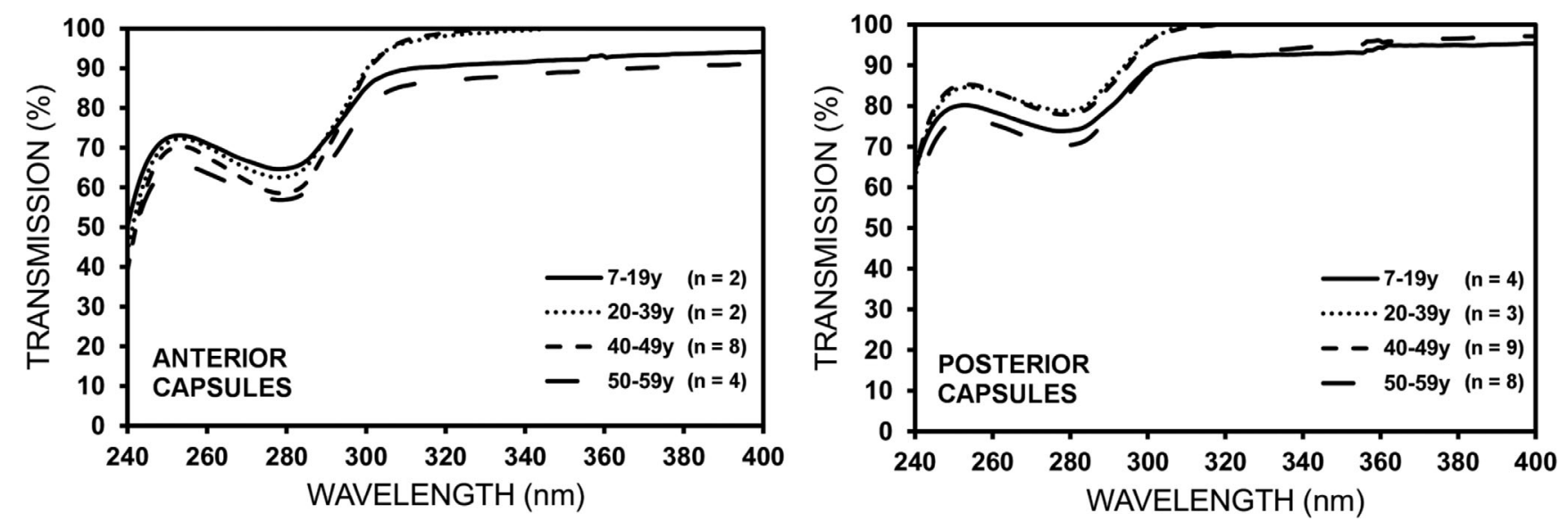

Figure 10. Calculated transmission of the lens capsules in four age groups. Number of available samples in the related group are indicated in brackets.

light, respectively. Older anterior capsules are able to transmit less light, presumably due to their increased thickness (31).

As the lens grows continuously, it is evident that the absorbance of the whole lens becomes higher with age. On the other hand, here, we demonstrated the age-dependent changes of the absorption coefficient which is a more characteristic parameter. The statistical analysis showed that the age affects significantly the absorption properties of the posterior layers while the changes of the anterior ones are less notable. Previously, it was found that the greater UV absorption in the posterior cortex is accompanied by higher levels of Alpha-crystallin A and Betacrystallin B1 chains (17) and alpha-crystallin was suggested to responsible for the greater UV absorbance in the posterior layers. As alpha-crystallin is a major target for UV irradiation due to its higher protein unfolding and as the unfolding increases with age (32), alpha-crystallin may play an important role in the significant correlation between absorption and age in the case of posterior lens sections. At longer wavelengths, only posterior nuclear layers showed age-dependent changes. These are presumably associated with the increased level of the bound UV-filters in the old nucleus.

These results suggest that the absorbance of the old lens is higher not only due to its greater thickness but also the increased absorption capacity of the posterior layers. Although the capsules show no age-related changes, the thicker anterior capsule also increases the total absorbance of the aging lens. These findings could help better understand the process of the age-related or UV-induced cataract formation. Data related to the absorption of lens capsules could be also important in cataract surgery and add considerable information to the design of intraocular lenses.

Acknowledgements-This study was supported by the European Union and the State of Hungary, cofinanced by the European Social Fund within the framework of EFOP-3.6.1-16-2016-00008.

\section{SUPPORTING INFORMATION}

Additional supporting information may be found online in the Supporting Information section at the end of the article:

Figure S1. Absorption coefficient of lens sections derived from a young (16 years old) and from an older lens (52 years old) in the UV-A and -B ranges. The absorption spectrum of the young lens presents a local minimum at $320 \mathrm{~nm}$ (left).
Figure S2. Absorption coefficients of individual lens sections. Note the high variability of the spectra of lenses with the same age.

Table S1. Standard deviation of absorption coefficients taken at $280 \mathrm{~nm}$ together with mean values in three age groups.

Table S2. Standard deviation of absorption coefficients taken at $360 \mathrm{~nm}$ together with mean values in three age groups.

\section{REFERENCES}

1. Weale, R. A. (1988) Age and the transmittance of the human crystalline lens. J. Physiol. 395, 577-587.

2. Gaillard, E. R., L. Zheng, J. C. Merriam and J. Dillon (2000) Agerelated changes in the absorption characteristics of the primate lens. Invest. Ophthalmol. Vis. Sci. 41, 1454-1459.

3. Artigas, M. A., A. Felipe, A. Navea, A. Fandino and C. Artigas (2012) Spectral transmission of the human crystalline lens in adult and elderly persons: Color and total transmission of visible light. Invest. Ophthalmol. Vis. Sci. 53, 4076-4084.

4. Zigman, S., T. Paxhia, T. McDaniel, M. F. Lou and N. T. Yu (1991) Effect of chronic near-ultraviolet radiation on the gray squirrel lens in vivo. Invest. Ophthalmol. Vis. Sci. 32, 1723-1732.

5. Zigman, S. (1993) Ocular light damage. Photochem. Photobiol. 57, 1060-1068.

6. Merriam, J. C. (1996) The concentration of light in the human lens. Trans. Am. Ophthalmol Soc. 94, 803-918.

7. Varma, S. D., S. Kovtun and K. R. Hegde (2011) Role of UV irradiation and oxidative stress in cataract formation. Medical prevention by nutritional antioxidants and metabolic agonists. Eye. Contact. Lens. 37, 233-245.

8. Hollows, F. and D. Moran (1981) Cataract - the ultraviolet risk factor. Lancet 2, 1249-1250.

9. Bochow, T. W., S. K. West, A. Azar, B. Munoz, A. Sommer and H. R. Taylor (1989) Ultraviolet light exposure and risk of posterior subcapsular cataracts. Arch. Ophthalmol. 107, 369-372.

10. Cruickshanks, K. J., B. E. Klein and R. Klein (1992) Ultraviolet light exposure and lens opacities: The beaver dam eye study. Am. J. Public. Health. 82, 1658-1662.

11. Dolin, P. J. (1994) Ultraviolet radiation and cataract: a review of the epidemiological evidence. Br. J. Ophthalmol. 78, 478-482.

12. Klein, B. E. K., R. Klein and K. E. Lee (1998) Incidence of age-related cataract. Arch. Ophthlamol. 116, 219-225.

13. AREDS Research Group (2001) Risk factors associated with age-related nuclear and cortical cataract. Ophthalmology 108, 1400-1408.

14. Boettner, E. A. and J. M. Wolter (1962) Transmission of the ocular media. Invest. Ophthalmol. Vis. Sci. 1, 776-783.

15. Mellerio, J. (1987) Yellowing of the human lens: Nuclear and cortical contributions. Vision. Res. 27, 1581-1587. 


\section{Viktor Pajer et al.}

16. Dillon, J., L. Zheng, J. C. Merriam and E. R. Gaillard (1999) The optical properties of the anterior segment of the eye: Implications for cortical cataract. Exp. Eye Res. 68, 785-795.

17. Pajer, V., Á. Tiboldi, N. Bae, K. Li, S. U. Kang, B. Hopp, L. Kolozsvári, G. Lubec and A. Nógrádi (2013) The molecular background of the differential UV absorbance of the human lens in the 240- to 400nm range. Photochem. Photobiol. 89, 856-863.

18. Murata, Y. (1987) Light absorption characteristics of the lens capsule. Ophthalmic. Res. 19, 107-112.

19. Taylor, V. L., A. J. Al-Ghoul, C. W. Lane, V. A. Davis, J. R. Kuszak and M. J. Costello (1996) Morphology of the normal human lens. Invest. Ophthalmol. Vis. Sci. 37, 1396-1410.

20. Augusteyn, R. C. (2010) On the growth and internal structure of the human lens. Exp. Eye. Res. 90, 643-654.

21. Rosen, A. M., D. B. Denham, V. Fernandez, D. Borja, A. Ho, F Manns, J. P. Parel and R. C. Augusteyn (2006) In vitro curvatures and dimensions of human lenses. Vision. Res. 46, 1002-1009.

22. Cooper, G. F. and J. G. Robson (1969) The yellow colour of the lens of men and other primates. J. Physiol. 203, 411-417.

23. Moffat, B. A. and J. M. Pope (2002) The interpolation of multi-exponential water proton transverse relaxation in the human and porcine eye lens. Magn. Reson. Imaging. 20, 83-89.

24. Siebinga, I., G. F. J. M. Vrensen, F. F. M. De Mul and J. Greve (1990) Age-related changes in local water protein content of human eye lenses measured by Raman microspectroscopy. Exp. Eye. Res. 53, 233-239.
25. Bron, A. J., G. F. J. M. Vrensen, J. Koretz, G. Maraini and J. J. Harding (2000) The ageing lens. Ophthalmologica. 214, 86104.

26. Zelentsova, E. A., L. V. Yanshole, A. Z. Fursova and Y. P. Tsentalovich (2017) Optical properties of the human lens constituents. $J$. Photoch. Photobio B. 173, 318-324.

27. Bova, L. M., M. H. J. Sweeney, J. F. Jamie and R. J. W. Truscott (2001) Major changes in human ocular UV protection. Invest. Ophthalmol. Vis. Sci. 42, 200-205.

28. Korlimbinis, A., J. A. Aquilina and R. J. W. Truscott (2007) Protein-bound UV filters in normal human lenses: The concentration of bound UV filters equals that of free UV filters in the center of older lenses. Invest. Ophthalmol. Vis. Sci. 48, 1718-1723.

29. Lerman, S. (1987) Chemical and physical properties of the normal and aging lens: spectroscopic (UV, fluorescence, phosphorescence, and NMR) analyses. Am. J. Optom. Phys. Opt. 64, 11-22.

30. Ambach, W., M. Blumthaler, T. Schöpf, E. Ambach, F. Katzgraber, F. Daxecker and A. Daxer (1994) Spectral transmission of the optical media of the human eye with respect to keratitis and cataract formation. Doc. Ophthalmol. 88, 165-173.

31. Krag, S. and T. T. Andreassen (2003) Mechanical properties of the human posterior lens capsule. Invest. Ophthalmol. Vis. Sci. 44, 691696.

32. Liang, J. N., S. K. Bose and B. Chakrabarti (1985) Age-related changes in protein conformation in bovine lens crystallins. Exp. Eye. Res. 40, 461-469. 